\title{
Histone Hypervariants H2A.Z.1 and H2A.Z.2 Play Independent and Context-Specific Roles in Neuronal Activity-Induced Transcription of Arc/ Arg3.1 and Other Immediate Early Genes
}

\author{
Carissa J. Dunn,, ${ }^{1,}$ Pushpita Sarkar,, ${ }^{1,}$ Emma R. Bailey, ${ }^{2}$ 'Shannon Farris, ${ }^{2}$ Meilan Zhao, ${ }^{2}$ (D) James M.
} Ward, ${ }^{3}$ (D) Serena M. Dudek, ${ }^{2}$ and ${ }^{-}$Ramendra N. Saha ${ }^{1}$

\section{DOI:http://dx.doi.org/10.1523/ENEURO.0040-17.2017}

${ }^{1}$ Molecular and Cell Biology Unit, School of Natural Sciences, University of California at Merced, Merced, CA 95343, ${ }^{2}$ Laboratory of Neurobiology, NIEHS, NIH, Research Triangle Park, NC 27709, and ${ }^{3}$ Integrative Bioinformatics Support Group, NIEHS, NIH, Research Triangle Park, NC 27709

\begin{abstract}
The histone variant $\mathrm{H} 2 \mathrm{~A} . \mathrm{Z}$ is an essential and conserved regulator of eukaryotic gene transcription. However, the exact role of this histone in the transcriptional process remains perplexing. In vertebrates, H2A.Z has two hypervariants, H2A.Z.1 and H2A.Z.2, that have almost identical sequences except for three amino acid residues. Due to such similarity, functional specificity of these hypervariants in neurobiological processes, if any, remain largely unknown. In this study with dissociated rat cortical neurons, we asked if H2A.Z hypervariants have distinct functions in regulating basal and activity-induced gene transcription. Hypervariant-specific RNAi and microarray analyses revealed that H2A.Z.1 and H2A.Z.2 regulate basal expression of largely nonoverlapping gene sets, including genes that code for several synaptic proteins. In response to neuronal activity, rapid transcription of our model gene Arc is impaired by depletion of H2A.Z.2, but not H2A.Z.1. This impairment is partially rescued by codepletion of the H2A.Z chaperone, ANP32E. In contrast, under a different context (after $48 \mathrm{~h}$ of tetrodotoxin, TTX), rapid transcription of Arc is impaired by depletion of either hypervariant. Such context-dependent roles of H2A.Z hypervariants, as revealed by our multiplexed gene expression assays, are also evident with several other immediate early genes, where regulatory roles of these hypervariants vary from gene to gene under different conditions. Together, our data suggest that H2A.Z hypervariants have context-specific roles that complement each other to mediate activity-induced neuronal gene transcription.
\end{abstract}

Key words: Arc; epigenetics; H2A.Z; H2A.Z.1; H2A.Z.2; histone

\section{Significance Statement}

Epigenetic regulation of activity-induced gene transcription is pivotal in mediating neuronal responses that underlie several transcription-dependent downstream brain processes, yet it remains poorly understood. Thus, understanding roles of such epigenetic processes and their core components, such as variant histone $\mathrm{H} 2 \mathrm{~A} . \mathrm{Z}$, is necessary to comprehend brain development and function. In vertebrates, H2A.Z has two hypervariants, H2A.Z.1 and H2A.Z.2, which are encoded by different genes and differ by only three amino acids. Despite such similar sequences, we provide evidence suggesting that they regulate nonoverlapping gene cohorts in neurons and play context-specific, nonredundant roles in activity-induced transcription of immediate early genes. Together, our findings represent a substantial departure from the current H2A.Z biology by shifting the focus to independent contributions of H2A.Z hypervariants. 


\section{Introduction}

Activity-induced gene transcription, an integral component of neural plasticity, is conducted at the chromatin level by enabling the transcription machinery to access and "read-out" DNA (Greer and Greenberg, 2008). Several activity-induced neuronal immediate early genes (IEGs) are accessed independent of signal by a transcriptionally engaged RNA polymerase II (Pol II) that pauses proximal to the promoter until an activity signal is received (Saha et al., 2011; Adelman and Lis, 2012). Activity-induced "turn-on" signals release the paused Pol II, which then efficiently transcribes the gene by running through spools of nucleosomes (productive elongation). Nucleosomes, the fundamental chromatin unit, regulate Pol II elongation by acting as either barriers or facilitators (Owen-Hughes and Gkikopoulos, 2012; Teves et al., 2014). Such activitydependent nucleosomal role selection primarily depends on post-translational modifications of the histone tails and nucleosomal remodeling, including turnover of different histone variants (Michod et al., 2012).

In mammals, three of the four canonical histones, $\mathrm{H} 2 \mathrm{~A}$, $\mathrm{H} 2 \mathrm{~B}$, and $\mathrm{H} 3$, have diversified into several variant histones with distinct amino acid sequences that can influence transcriptionally relevant nucleosomal properties and dynamics (Weber and Henikoff, 2014). Recently, a few studies have unveiled important roles of histone variants $\mathrm{H} 2 \mathrm{~A} . \mathrm{Z}$ and $\mathrm{H} 3.3$ in neuronal gene transcription underlying brain development and function. For example, H2A.Z, which is conserved from yeast to human and has been implicated in both transcriptional activation and repression (Soboleva et al., 2014), plays a role in memory consolidation and cerebellar development in the brain (Zovkic et al., 2014; Yang et al., 2016). Similarly, H3.3, which differs from canonical H3 histones by only four to five

Received February 3, 2017; accepted August 9, 2017; First published August 17, 2017.

The authors declare no competing financial interests.

Author contributions: C.J.D., P.S., S.M.D., and R.N.S. designed research; C.J.D., P.S., E.R.B., S.F., M.Z., and R.N.S. performed research; C.J.D., P.S., E.R.B., S.F., M.Z., J.M.W., and R.N.S. analyzed data; C.J.D., P.S., and R.N.S. wrote the paper.

This work was supported by the United States Department of Health and Human Services (HHS) National Institutes of Health (NIH) National Institute of Mental Health Grant R00MH096941 (to R.N.S.), the HHS NIH National Institute of Environmental Health Sciences Grant ES100221 (to S.M.D.), and by start-up funds from University of California Merced (R.N.S.). For the K99 phase of the R00 Award, this research was supported by the Intramural Research Program of the National Institute of Environmental Health Sciences, National Institutes of Health.

${ }^{*}$ C.J.D. and P.S. contributed equally to this work.

Acknowledgements: We thank Ms. A. Funk and Dr. B. Bennett for project assistance, the Saha lab members for critical reading of this manuscript, and Dr. Mike Cleary and Dr. Sergei Nechaev. We also thank the personnel in the Viral Vector and Molecular Genomics cores at National Institute of Environmental Health Sciences, and the Stem Cell Instrumentation Foundry at University of California Merced for use of their facility.

Correspondence should be addressed to Ramendra N. Saha, Ph.D., Room 346 S\&E Building 1, University of California, Merced, 5200 North Lake Road, Merced, CA 95343, E-mail: rsaha3@ucmerced.edu.

DOI:http://dx.doi.org/10.1523/ENEURO.0040-17.2017

Copyright (C) 2017 Dunn et al.

This is an open-access article distributed under the terms of the Creative Commons Attribution 4.0 International license, which permits unrestricted use, distribution and reproduction in any medium provided that the original work is properly attributed. amino acids (Filipescu et al., 2013), regulates embryonic and adult neuronal gene expression patterns, thereby playing an essential role in plasticity and cognition (Maze et al., 2015). This example of H3.3 illustrates how very small dissimilarities in sequences of two closely related histone proteins may produce large differences in neuronal gene transcription (Maze et al., 2014).

Similar small differences also exist within the H2A.Z histone class. In vertebrates, H2A.Z has two very closely related paralogs that have been referred to as "hypervariants": H2A.Z.1 (formerly H2A.Z) and H2A.Z.2 (formerly H2A.V; Talbert et al., 2012). Two nonallelic genes, H2afz and $H 2 a f v$, which are driven by independent promoters on different chromosomes, encode these H2A.Z hypervariants (Dryhurst et al., 2009; Matsuda et al., 2010). H2A.Z.1 and H2A.Z.2 differ by only three amino acids, which are located far apart on the polypeptide (Fig. 1A) and are structurally similar to their respective counterpart in that there are no usable difference in their antigenicity (e.g., hydrophobic vs hydrophilic, ring structure vs linear, or large vs small in residue size). Thus, there are currently no antibodies available to distinguish between H2A.Z.1 and H2A.Z.2. Due to this limitation, our understanding of H2A.Z is most likely a portmanteau of both hypervariant functions. Such a composite picture of their function would not be a concern if these hypervariants were functionally redundant and homogeneous (Soboleva et al., 2014). However, a few structural and functional features suggest the contrary. Recently resolved crystal structures of H2A.Z-containing nucleosomes revealed subtle differences in the H2A.Z.1 and H2A.Z.2 L1 loop (Horikoshi et al., 2013), but it remains unknown whether such differences manifest any functional consequences. In H2A.Z.1 ${ }^{-1-}$ mice, which are embryonic lethal (Faast et al., 2001), H2A.Z.2 is apparently unable to compensate for the loss of its twin isoform, thereby suggesting a unique role for H2A.Z.1 during early embryonic development. Similar functional inequality of H2A.Z.1 and H2A.Z.2 has also been recently reported in melanoma cells (Vardabasso et al., 2015). Taken together, there remains an untested possibility that these two H2A.Z hypervariants may not be functionally identical, but instead have discrete nonoverlapping roles (functional specificity) in gene transcription. We tested this hypothesis in neurons and report here that H2A.Z.1 and H2A.Z.2 have independent and contextspecific roles in basal and neuronal activity-induced gene transcription.

\section{Materials and Methods}

\section{Plasmids and subcloning}

Several web-based software programs were used to design eight (four each) hypervariant-specific shRNA for RNAi. The shRNA were inserted into pLKO.1-puro (designed by RNAi consortium or TRC; obtained from Addgene) following the protocol on the Addgene website. Self-inactivating HIV lentivirus particles were produced by transfecting 293T cells with the shRNA vector, envelope (pMD2.G; Addgene), and packaging plasmids (psPAX2; Addgene). For construction of exogenous tagged constructs, DNA sequences encoding HA-H2A.Z.1, HA.IS1, 
H2A.Z.1 AGGKA GKDSG KAKTK AVSRS QRAGL QFPVG RIHRH LKSR ...V L E L A G N A...KTV

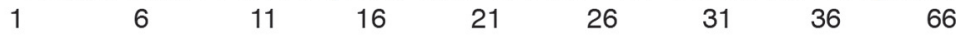

H2A.Z.2 AGGKA GKDSG KAKAK AVSRS QRAGL QFPVG RIHRH LKTR...V L E L A G N A...KTA

B

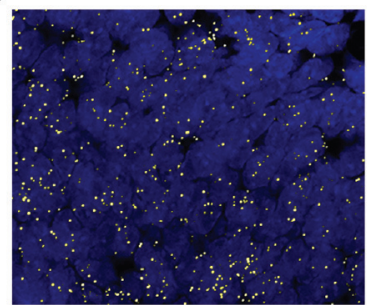

H2afz mRNA

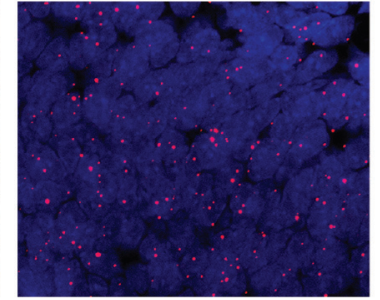

H2afv mRNA ...gtgttggagttggcaggtaatgct...

H2afv shRNA

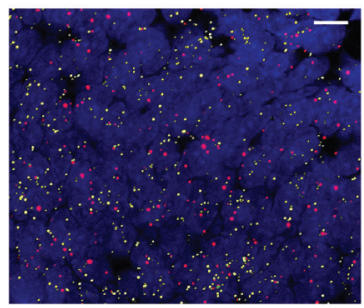

Merged

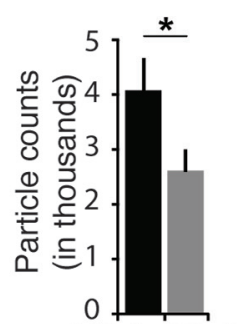

H2afz H2afv
C

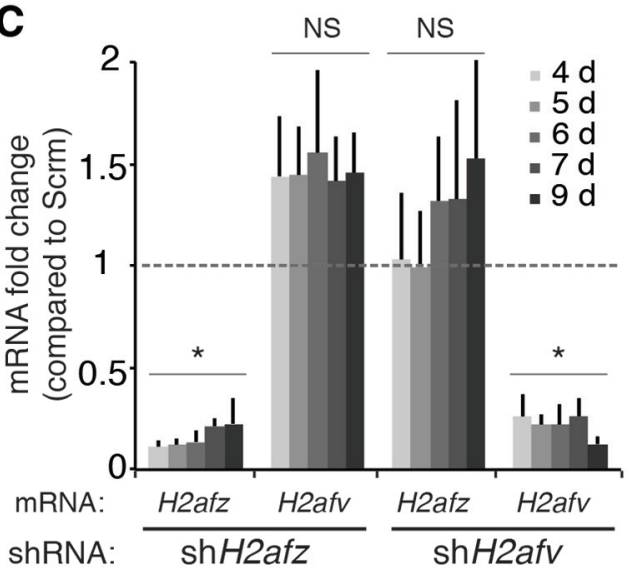

$\mathbf{F}$

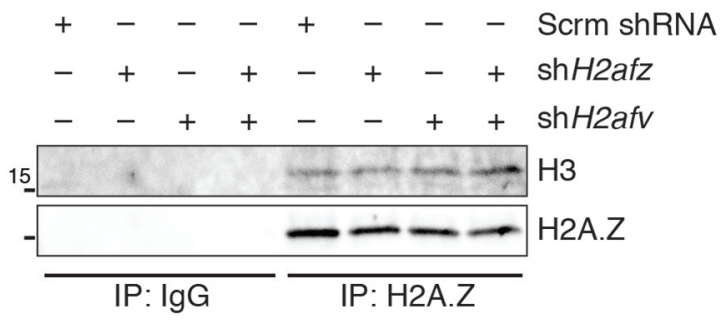

G
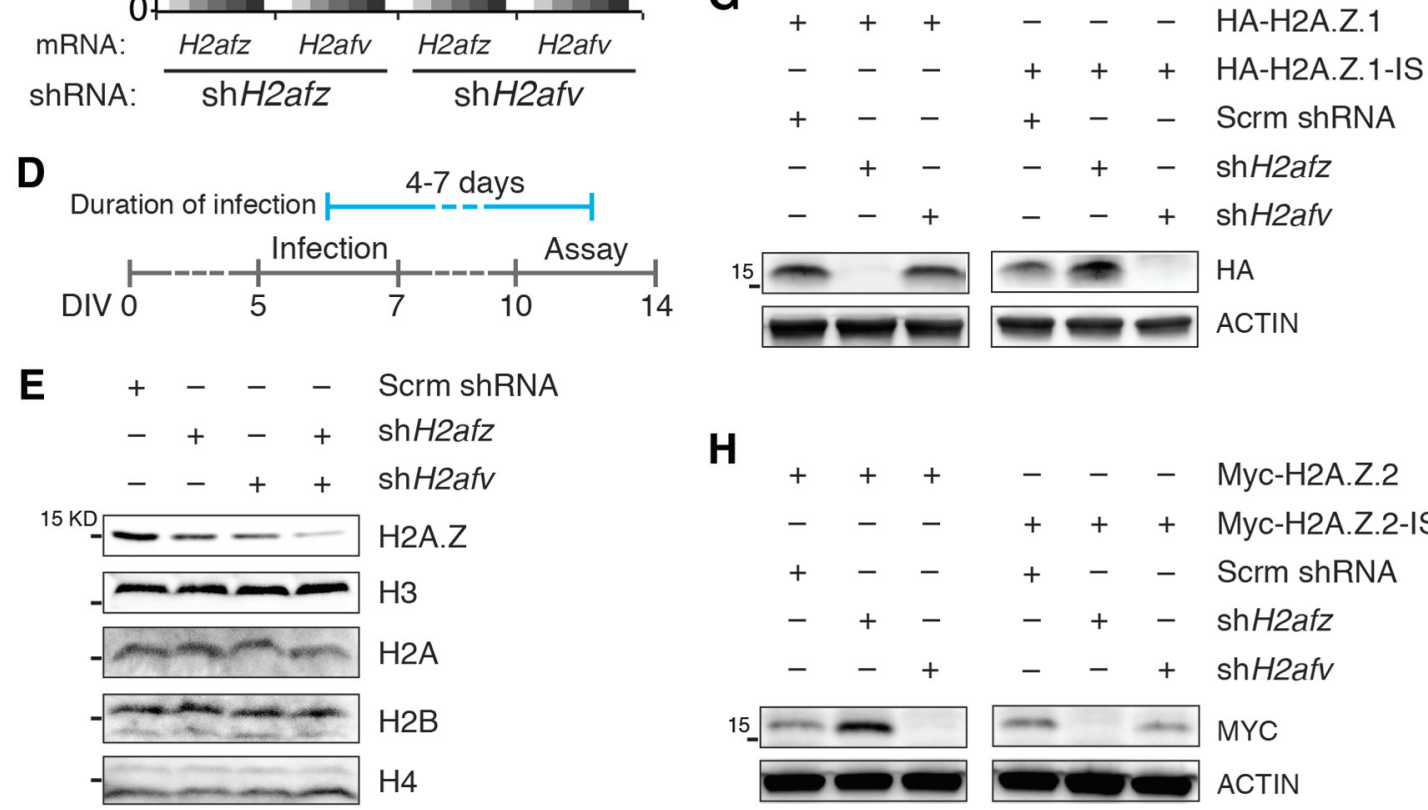

H

$+++-\quad-\quad$ Myc-H2A.Z.2

$-\quad-+++$ Myc-H2A.Z.2-IS

$+--+-\quad-$ Scrm shRNA

$-+-\quad+\quad-$ shH2afz

- $++\ldots+$ - shH2afv
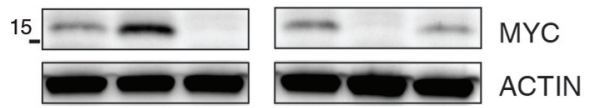

Figure 1. Validation of H2A.Z hypervariant-specific RNAi. A, H2A.Z.1 and H2A.Z.2 amino acid sequences are depicted where three different amino acid residues are indicated in red. The target sequences of shH2afz and shH2afv are noted in blue. $\boldsymbol{B}$, left, Representative images of smFISH of H2afv and H2afz in E18 rat cortex. Right, Quantification of H2afz and H2afv molecules; particle count per $212 \mu \mathrm{m}^{2} . N=3, * p<0.05$ (one-tailed unpaired $t$ test). Scale bar, $10 \mu \mathrm{m}$. C, Cortical neurons in culture were infected with lentiviruses delivering either shH2afz or shH2afv and total RNA was collected after indicated number of days. Compared to control neurons, changes in H2afz and H2afv mRNA levels were determined by qPCR, normalized to Gapdh mRNA levels (internal control) and are depicted here as fold change. NS, not significant. $* p<0.05 . N=3-6$. $\boldsymbol{D}$, Timeline of infection and treatment for all assays performed in dissociated cortical neurons. Cells were infected with lentiviruses between 5 and $7 \mathrm{~d}$ after plating (gray line), and assays were performed between 4 and $7 \mathrm{~d}$ after infection (between DIV10 and DIV14; blue line). $\boldsymbol{E}$, Total acid-extracted histone from neurons 


\section{continued}

infected with lentiviruses delivering either shH2afz or shH2afv or both for $6-7 \mathrm{~d}$, resolved by electrophoresis, and blotted for indicated histone. $N=3$. Note the cumulative effect of depleting both hypervariants when probed with total H2A.Z antibody. $F$, IP of nucleosomal H2A.Z from nuclear extract of neurons infected with lentiviruses delivering either shH2afz or shH2afv or both for $6 \mathrm{~d}$ resolved by electrophoresis. $N=3 . \boldsymbol{G}, \boldsymbol{H}$, Neurons were coinfected with indicated constructs for $4-5 \mathrm{~d}$ and nuclear extracts were resolved by electrophoresis. IS, insensitive; shRNA target regions were swapped in these constructs. $N=3$.

HA-ISB, MYC-H2A.Z.2, MYC-IS2, and MYC-ISB were generated each with 5' EcoRl and 3' Notl restriction sites, using Life Technologies GeneArt Gene Synthesis "strings." These EcoRI-Notl DNA fragments were cloned into the System Biosciences lentiviral plasmid CD811A-1, using the corresponding restriction sites on the vector. Subsequently, DNA constructs were characterized by restriction mapping using EcoRl and Notl enzymes (New England Biolabs), and inserted elements were validated by sequencing. These constructs were packaged into lentiviruses as mentioned above.

\section{Dissociated neuronal culture, RNAi, and cell treatment}

Cultures of cortical neurons were prepared from embryonic day 18 (E18) Sprague Dawley rats of either sex. All animal procedures were performed in accordance with the National Institute of Environmental Health Sciences (NIEHS) and the University of California Merced animal care committee's regulations [NIEHS Institutional Animal Care and Use Committee (IACUC) approval: ASP\#01-21; and University of California Merced IACUC approval: ASP\#13-0007 and ASP\#16-0004]. Dissociated cortical neurons were plated in Neurobasal medium (Invitrogen) supplemented with $25 \mu \mathrm{M}$ glutamate (Sigma-Aldrich), 0.5 mM L-glutamine (Sigma-Aldrich), either B27 (Invitrogen) or NS21, and maintained in a similar medium without the glutamate. NS21 was prepared in the laboratory as previously described (Chen et al., 2008). Neurons were routinely used between 10 and $14 \mathrm{~d}$ in vitro (DIV), a stage when synapses have formed and are effective. For infection with recombinant lentiviruses, the viral supernatant was diluted in neuronal media and cells were infected between DIV5 and DIV7 at a multiplicity of infection ranging from 2 to 5 for $4-7 \mathrm{~d}$. To induce gene transcription under resting conditions using synaptic circuits, we cotreated neurons with $50 \mu \mathrm{M}$ bicuculline (Bic; Sigma-Aldrich) and $75 \mu \mathrm{M}$ 4-aminopyridine (4AP; Acros Organics; Papadia et al., 2005). To induce gene transcription extrasynaptically, we blocked neuronal activity with 1-2 $\mu \mathrm{M}$ tetrodotoxin (TTX; Calbiochem) and activated the MAP kinase (MAPK) pathway (via PKC) with $1 \mu \mathrm{M}$ phorbol ester myristate (PMA; Sigma-Aldrich; Schultz et al., 1997). To induce gene transcription at the intranuclear level, neurons were treated with $100 \mu \mathrm{M} 5$, 6-dichloro-1- $\beta$-Dribofuranosyl-benzimidazole (DRB; Sigma-Aldrich) alone, or in combination with 250-500 nM triptolide (Trip; Tocris) for $1 \mathrm{~h}$ followed by washout with preconditioned media. To induce genes after homeostatic changes, a previously described protocol using prolonged treatment of TTX for $48 \mathrm{~h}$ followed by its washout was used (Saha et al., 2011).

\section{Electrophysiological recordings}

Whole-cell patch-clamp recordings were performed on dissociated cortical neurons coexpressing GFP and shRNA by an experimenter blinded to the identity of the treatment. Miniature EPSCs (mEPSCs) were assessed for frequency and amplitude as detailed previously (Saha et al., 2011). For visualization of infected neurons using GFP coexpression, the shRNA component was subcloned from the pLKO.1 to the pLKO.3G backbone as detailed previously (Saha et al., 2011). To ensure adequate knockdown, PCR was performed in sister neuronal cultures with the same batch of viruses to confirm their efficacy.

\section{RNA extraction and gene transcription quantification}

Total RNA was isolated from dissociated neurons using the RNeasy Mini kit (QIAGEN) with in-column DNase (QIAGEN) digestion. cDNA was synthesized using MuLV reverse transcriptase (Promega), random primers (Promega), oligo dTs (Promega), and RNase inhibitors (Thermo Scientific). qPCR was performed using iTaq Universal Sybr Green Supermix (Bio-Rad) and the Bio-Rad CFX Connect real-time PCR Detection System. Pre-mRNA was estimated as described previously (Saha, et al., 2011). Rat PCR primers used in this study are listed in Table 1.

\section{Expression microarray and data analysis}

Gene expression analysis was performed using RNA obtained from four biological replicates. The Agilent Whole Rat Genome 4x44 multiplex format oligo arrays (014879, Agilent Technologies) were used following the Agilent 1-color microarray-based gene expression analysis protocol. Using $500 \mathrm{ng}$ of total RNA, Cy3-labeled cRNA was produced according to the manufacturer's protocol. For each sample $1.65 \mu \mathrm{g}$ of Cy3-labeled cRNAs were fragmented and hybridized for $17 \mathrm{~h}$ in a rotating hybridization oven. Slides were washed and then scanned with an Agilent Scanner. Data were obtained using the Agilent Feature Extraction software (v9.5), using the 1-color defaults for all parameters. The Agilent Feature Extraction Software performed error modeling, adjusting for additive and multiplicative noise. Then, "gProcessedSignal" values were extracted for each probe and sample and used as the baseline expression measurements and quantile normalization was performed to normalize the samples. Probe-based expression data were collapsed down to a single measurement per gene by taking the median of all probes associated with each gene. Next, the average expression was calculated across all samples for each gene and the bottom $10 \%$ of genes with the lowest average expression was removed. Differentially expressed genes (DEGs) were identified by first performing a two-sample 
Table 1. List of primers used in the study

\begin{tabular}{ll}
\hline Target & Forward primer \\
Arc pre-mRNA & GAATTTCTATGCCAACTCACGGG \\
Btg2 pre-mRNA & CTCTCTCTCTTGTTTCCTCCACAG \\
cFos pre-mRNA & ACAGCCTTCCTACTACCATTCCC \\
Cyr61 pre-mRNA & ATGTATGAGTTTCAGCGTGTGGCG \\
Dusp1 pre-mRNA & CTCTACGACCAGGTTAGTAGGAGT \\
Dusp6 pre-mRNA & TCCTGTGCCTCTCACAAGCTGAAA \\
Fbxo33 pre-mRNA & GCATCTACTTGGAGCTGGTGTTGT \\
Gadd45g pre-mRNA & ACTCACGGCGCTTGTCTTTCACA \\
GAPDH pre-mRNA & AACATGCACAGGGTACTTCGAGGA \\
Npas4 pre-mRNA & GTTGCATCAACTCCAGAGCCAAGT \\
Nr4a3 pre-mRNA & ATGGAGTGTCAACTGGCTTCTGAG \\
GAPDH mRNA & AGAGACAGCCGCATCTTCTTG \\
H2Afz mRNA & GAAGAAAGGACAACAGAAGACTGT \\
H2Afv mRNA & ACCCTATGCTCCCGTGTGTTAGAA \\
18 s rRNA & CATTCGAACGTCTGCCCTAT \\
ANP32e mRNA & TCAGAAGTAGGAGAGGGAGAAG
\end{tabular}

Reverse primer

AGTCATGGAGCCGAAGTCTGCTTT

TGTGGTTGATGCGGATACAGCGAT CTGCACAAAGCCAAACTCACCTGT GTCTGCCTTCTGACTGAGCTGTAA ACAGCCGCTTTCTCTATTCTCCCT AACTTACTGAAGCCACCTGCCAGA TCCACGCAAGCCTACCTGTTGTT ATTCAGGACTTTGGCGGACTCGTA ACGACATACTCAGCACCAGCATCA ACATTTGGGCTGGACCTACCTTCA GCCATAAGTCTGCGTGGCATAAGT GGTAACCAGGCGTCCGATAC CAGCTGTTAAGAGTATTTAGAGTCC AGGCAAAGATCAGCACCAACTCTG GTTTCTCAGGCTCCCTCTCC CCTTGGAGGGTCTAATCATCATC

Student's $t$ test for each gene followed by Benjamini Hochberg correction. Genes were defined as differentially expressed if they had $p<0.001$ and a fold change of 1.25 or greater. For the heat map depicting expression of these DEGs, the expression values were normalized to have a mean of zero and a standard deviation of one, and hierarchical clustering of these genes was performed. GO terms that were enriched for these DEGs were identified using the online tool DAVID (http://david.ncifcrf.gov/). GEO accession number for these datasets is GSE96886.

\section{Sample preparation for electrophoresis}

Neurons were lysed in ice-cold $1 \times$ RIPA buffer $(25 \mathrm{mM}$ Tris, $\mathrm{pH} 7.5,150 \mathrm{mM} \mathrm{NaCl}, 1 \%$ Na-deoxycholate, $0.1 \%$ SDS, and 1\% NP-40) and supplemented with 1:100 protease inhibitor cocktail (Sigma-Aldrich). Lysed neurons were sheared by sonication, cell debris pelleted at 15000 rpm for 5 min at $4^{\circ} \mathrm{C}$, and the clarified supernatant transferred to a prechilled $1.7 \mathrm{ml}$ microcentrifuge tube. Total cell extracts were denatured at $95^{\circ} \mathrm{C}$ for $5 \mathrm{~min}$, using either home-made $5 \times$ Laemmli buffer, BOLT $4 \times$ Sample buffer (Life Technologies) and BOLT 10× reducing agent (Life Technologies), $2 \times-$, or $4 \times$ Laemmli sample buffer (both from Bio-Rad). To obtain synaptic fractions, cell pellets were treated with $1 \mathrm{ml}$ ice-cold Syn-PER reagent (Thermo Scientific catalogue number 87793) and supplemented with 1:100 protease inhibitor cocktail (SigmaAldrich). Neurons were incubated on ice for 10 min, gently triturated 10 times, centrifuged at $1200 \times g$ for $10 \mathrm{~min}$ at $4^{\circ} \mathrm{C}$, and supernatant was transferred to ice-cold microcentrifuge tubes, then recentrifuged at $15,000 \times g$ for 20 $\min$ at $4^{\circ} \mathrm{C}$. The pellet was then resuspended in $20 \mu \mathrm{l} 2 \times$ Laemmli sample buffer (Bio-Rad). Acid-extracted histone fractions were prepared using a protocol described previously (Shechter et al., 2007). Histone pellets were denatured using $2 \times$ Laemmli sample buffer (Bio-Rad).

\section{Immunoprecipitations (IPs)}

All IPs were performed overnight, at $4^{\circ} \mathrm{C}$ in $1 \times$ IP buffer (0.5\% Triton X-100, 0.002 M EDTA, $0.02 \mathrm{M}$ Tris $\mathrm{pH}$ 7.75, $0.15 \mathrm{M} \mathrm{NaCl}$, and $10 \%$ glycerol), or in $1 \times$ IP buffer from the SimpleChIP Enzymatic Chromatin IP kit (Cell Signaling
Technology catalogue number 9003). Proteins were isolated from overnight IPs by incubating with Pierce Protein A/G Magnetic Beads (catalogue number 88803) for $1 \mathrm{~h}$ at $4^{\circ} \mathrm{C}$. Subsequently, the beads were washed three times with IP buffer, denatured, separated by electrophoresis, and immune blotted as noted below.

\section{Western blotting and imaging}

Denatured protein samples were resolved on $4-20 \%$ (Bio-Rad catalogue number 4568095), 4-15\% (Bio-Rad catalogue number 456-1083), 8-16\% (Bio-Rad catalogue number 456-1103) Mini PROTEAN gels, or on BOLT 4-12\% Bis-Tris Plus Gels (Life Technologies catalogue number NW04122BOX), in Tris/Glycine/SDS (Bio-Rad catalogue number 1610772), or $1 \times$ MOPS buffer (Life Technologies catalogue number B0001), respectively. Resolved proteins were transferred onto LF PVDF membrane, using the Bio-Rad TBT RTA kit and protocol (catalogue number 1704272). PVDF membranes were incubated at $4^{\circ} \mathrm{C}$ overnight with appropriate primary antibodies in $1 \times$ TBS-T with $0.5 \%$ BSA. The next day, membranes were washed three times in $1 \times$ TBS-T, probed with appropriate Alexa Fluor secondary antibodies (Life Technologies) for 30-45 min at room temperature, washed three times with $1 \times$ TBS-T, and imaged using Bio-Rad ChemiDoc Imaging System.

\section{Chromatin digestion}

Micrococcal nuclease (MNase) digests of rat dissociated neurons were performed using the SimpleChIP Enzymatic Chromatin IP kit (Cell Signaling Technology catalogue number 9003). Cells were fixed using Thermo Scientific Pierce methanol-free formaldehyde (catalogue number PI28906). The manufacturer's protocol was used with the exception that Thermo Scientific Pierce Micrococcal Nuclease (catalogue number PI88216) was used in place of CST MNase. Postdigest, samples were briefly sonicated at low settings to rupture the nucleus and then were either subjected to chromatin IP (ChIP), DNA extraction, or protein co-IP following the CST kit protocol. 


\section{ChIP}

Neurons were fixed with formaldehyde for 5-10 min, lysed in the sonication buffer (20 mM Tris, $\mathrm{pH} 7.8,2 \mathrm{mM}$ EDTA, $0.5 \mathrm{mM}$ EGTA, 0.5\% SDS, and inhibitors for proteases and phosphatases), and then sonicated for sixteen cycles (bursts and intervals of $30 \mathrm{~s}$ each) with the Bioruptor (Diagenode) to obtain 200- to 1000-bp genomic DNA fragments. A fraction of the sonicated sample was then immunoprecipitated overnight at $4^{\circ} \mathrm{C}$ in the $1 \times$ IP buffer with 2- to 5- $\mu$ g antibody. Antigen-antibody complexes were immunoprecipitated with Pierce Protein A/G Magnetic Beads, washed once with low salt buffer, three times with high salt buffer, once with $\mathrm{LiCl}$ buffer, and once with Tris-EDTA buffer. Samples were reverse cross-linked at $65^{\circ} \mathrm{C}$ overnight, and chromatin DNA was eluted using the QIAquick Nucleotide Removal kit (QIAGEN). Eluted chromatin was quantified by qPCR.

\section{Immunocytochemistry and microscopy}

Infected neurons were washed twice with $1 \times$ ice-cold PBS (Fisher Scientific). The cells were then incubated with $4 \%$ paraformaldehyde (Sigma-Aldrich) in $1 \times$ PBS for 15 min at room temperature and then washed twice with $1 \times$ PBS, permeabilized at room temperature for 20 min with $0.5 \%$ Triton X-100 (Fisher Scientific), washed twice with $1 \times$ PBS, and blocked for 30 min with $10 \%$ goat serum (Gibco) in $1 \times$ PBS. Cells were incubated at $4^{\circ} \mathrm{C}$ overnight in $3 \%$ goat serum in $1 \times$ PBS with primary antibodies at 1:500 dilution. The next day, the primary antibody solution was removed, and cells were washed three times with $0.05 \%$ Tween (Fisher) in $1 \times$ PBS (0.05\% PBS-T), and incubated with appropriate secondary antibody in 3\% goat serum in $1 \times$ PBS for $45 \mathrm{~min}$, washed three times with $0.05 \%$ PBS-T, and cured overnight using ProLong Anti-Fade Gold with DAPI (Life Technologies). Images were captured with a Keyence BZ9000-E microscope at $20 \times$ magnification.

\section{Antibodies}

Sheep anti-H2A.Z (Millipore, 09-862; RRID:AB_1587118), mouse anti-MYC (ThermoFisher Scientific, R95025; RRID: AB_2556560), rabbit anti-H3 (Cell Signaling Technology, 2650S; RRID:AB_2115124), mouse anti- $\beta$-ACTIN (ThermoFisher Scientific, AM4302; RRID:AB_2536382), rabbit antiARC (Synaptic Systems, 156003; RRID:AB_887694), rabbit anti-p-p44/42 MAPK (T202/Y204; Cell Signaling Technology, 4370S; RRID:AB_2315112), mouse anti-p44/42 MAPK (Erk1/2; L34F12; Cell Signaling Technology, 4696S; RRID: AB_390780), rabbit anti-HA (Cell Signaling Technology, 3724S; RRID:AB_1549585), mouse anti-MYC (Cell Signaling Technology, 2276S; RRID:AB_331783), rabbit anti-H2A (Cell Signaling Technology, 12349S; RRID:AB_2687875), mouse anti-H2B (Cell Signaling Technology, 2934S; RRID: AB_2295301), mouse anti-H4 (Cell Signaling Technology, 2935S; RRID:AB_1147658), rabbit anti-H2A.Z (Cell Signaling Technology, 2718S; RRID:AB_10694716), mouse anti-PSD95 (Antibodies Incorporated, 75-028; RRID:AB_2292909), rabbit anti-SH3 and multiple ankyrin repeat domains 3 (SHANK3) (Santa Cruz Biotechnology, SC30193; RRID:AB_2301759), goat anti-SHANK3 N16 (Santa Cruz Biotechnology, SC23547; RRID:AB_2187727), mouse anti-GluR2 (anti-
GluA2; Millipore, MAB397; RRID:AB_2113875), mouse antiHOMER1 (Synaptic Systems, 160011; RRID:AB_2120992), mouse anti-Synapsin1 (Synaptic Systems, 106001; RRID: AB_887805), rabbit anti-RPB1 (Cell Signaling Technology, 14958S; RRID:AB_2687876), rabbit anti-acetyl-H2A.Z Lys 5,7,11 (Millipore, ABE1363; RRID:AB_2687877), and rabbit anti-pSer5-RPB1 (Active Motif, 61085; RRID:AB_2687451).

\section{Single molecule fluorescent in situ hybridization (smFISH)}

Rat brains were harvested at E18 and individually embedded in $22 \times 22 \mathrm{~mm}$ cryomolds using optimal cutting temperature compound (OCT) and flash frozen in 2-methylbutane, which was cooled to $-20^{\circ} \mathrm{C}$ on a dry ice/ethanol slurry. Brains were sectioned on a Leica cryostat at $20 \mu \mathrm{m}$ and processed for single-molecule FISH according to the RNAscope Fluorescent Multiplex kit instructions (Advanced Cell Diagnostics). The following probes were used with the RNAscope fluorescent multiplex reagent kit: $\mathrm{Rn}-\mathrm{H} 2 \mathrm{afv}$ (20 ZZ probes targeting 4141815 of NM_001106019.1; part\# 495451), Rn-Hz1fz-C2 (13 ZZ probes targeting 12-792 of NM_022674.1; part\# 495441-C2), 3-plex Positive Control Probe, rat (part\# 320891), and 3-plex Negative Control Probe (part\# 320871). Images were acquired on a Zeiss 880 confocal microscope using a $40 \times$ oil-immersion lens. The image acquisition parameters were set using 3-plex negative controls (cDNA probes against bacterial RNAs not present in rat tissue) in each of the three channels (fluorescein isothiocyanate, CY3 and CY5) so that any signal above the level of background was acquired. At least three $212 \times 212 \mu \mathrm{m}$ $z$-stack images (1- $\mu \mathrm{m}$ steps) of somatosensory cortex were acquired per rat. Images were processed and analyzed using FIJI software (NIH v.2). Maximum intensity projection images were thresholded to an average value per channel and particles were counted using the analyze particle function in FIJl. Background particle counts were subtracted from each value before taking the average particle count per channel per rat $(N=3)$.

\section{Nanostring assay and data analysis}

NanoString probes were designed for indicated premRNAs by NanoString technologies and assays were performed following the manufacturer's protocol. Data were processed in $R$ (version 3.3.2), using the limma package (version 3.30.8) from Bioconductor (version 3.4). Data were log2 transformed, then batch-adjusted within each experiment and time point to account for sample processing errors. The pre-mRNA measurements were normalized using pre-mRNA housekeeper genes. Sample quality and processing were reviewed and confirmed using MA-plots and heat maps at each step. Pairwise comparisons were performed using the limma-voom methodology as previously described. Statistical hits were defined by requiring one sample group mean log2 signal at least 2 , absolute fold change above 1.5, and Benjamini Hochberg adjusted $p<0.01$. Heat maps were generated using group mean log2 normalized abundances, centered to the indicated control group mean. Violin plots were generated using the same values, centered to the treated scrambled for the respective group of 
treatments. Gene class changes per experiment and time point were tested using group mean values in a nonparametric, paired Wilcoxon test, with gene as the pairing factor, to test for coordinated gene changes between treated shH2A.Z.1 or treated shH2A.Z.2 and the corresponding treated scrambled of the same experiment time point.

\section{Experimental replicates and statistics}

Biological and technical replicates for each figure are indicated as $N$ and $n$, respectively, in corresponding figure legends. Error bars represent standard error of mean throughout this article. Statistical comparison of datasets was performed with the two-tailed Student's $t$ test (with Bonferroni corrections for multiple comparisons), twoway ANOVA, or Wilcoxon paired nonparametric test, unless otherwise stated in the figure legend.

\section{Results}

\section{H2A.Z hypervariants and strategy to target them independently}

First, we asked if both H2A.Z isoforms, H2A.Z.1 and H2A.Z.2 (Fig. 1A), are expressed in the brain. Using the smFISH approach and specific probes that bind to H2afz or H2afv, we detected both H2A.Z isoforms in the E18 rat brain (Fig. 1B). While there were significantly more H2afz particles compared to H2afv, we observed that the majority of DAPI-positive cells expressed both RNAs. Further studies were performed in dissociated cells obtained from E18 rat brains.

Next, we used RNAi to target these hypervariants independently. Both genes have been duplicated during evolution and have multiple mammalian "gene copies" that have variable noncoding regions despite having the same coding region. For example, in rat, when compared to the annotated H2afz on chromosome 2, an unnamed "pseudogene" on chromosome 16 (rat rn6 location: 7143846171439260) codes for an mRNA that retains the H2afz coding sequence in totality amid dissimilar noncoding regions. Therefore, to ensure knockdown of these probable pseudogene mRNAs as well, the coding region was targeted for RNAi. Initially, we designed four shRNA against each of the H2afz and H2afv mRNA coding regions and evaluated their knockdown efficacy by qPCR. Only one of these four shRNA pairs efficiently knocked down each hypervariant independently; others knocked down both hypervariants to some degree. Interestingly, these efficient shRNAs against H2afz (shH2afz) and H2afv (shH2afv) targeted the same region on the respective mRNA (Fig. 1A). These 24-bp target regions on H2afz and H2afv mRNAs differ by only $5 \mathrm{bp}$. Because of such scant difference, we tested whether shH2afz and shH2afv were indeed specific for their respective hypervariant targets.

Lentivirus-mediated delivery of shH2afz to dissociated rat cortical neurons significantly knocked down $\mathrm{H} 2 \mathrm{afz}$, but not H2afv, mRNA at all tested times after viral infection (Fig. 1C). Similarly, H2afv, but not H2afz, mRNA was knocked down by shH2afv (Fig. 1C). We also noted that loss of one hypervariant was not compensated by significantly enhanced expression of the other hypervariant.
Because efficient knockdown was noted between days 4 and 7 after infection, we decided to use this timeline for the rest of our assays (Fig. 1D). At the protein level, as detected by a commercial anti-H2A.Z antibody that does not discriminate between hypervariants, infection with shH2afz or shH2afv viruses partially depleted endogenous H2A.Z levels without affecting the expression of other histones (Fig. 1E). Although we were unable to completely abolish H2A.Z signal on infection with both shH2afz and shH2afv viruses, H2A.Z protein depletion was more pronounced on coinfection. We suspect that the protein remaining after coinfection is nucleosomal H2A.Z protected in the heterochromatin (Fig. 1E,F). The cumulative effect of such coinfection suggests that neurons express both hypervariants, and that they are sensitive to their respective shRNA only.

To test the above stated possibility further, we coexpressed shH2afz or shH2afv with either tagged HAH2A.Z.1 or tagged Myc-H2A.Z.2. Protein expression of HA-H2A.Z.1 was attenuated by shH2afz, but not shH2afv. Similarly, MYC-H2A.Z.2 protein expression was sensitive to shH2afv, but not shH2afz (Fig. $1 G, H$, left panels). To test shH2afz and shH2afv specificity even further, we exchanged their 24-bp target sequence in our exogenous constructs with the expectation that shRNA sensitivity of these constructs will also be reversed. As expected, HAH2A.Z.1-IS, the H2afz mRNA with the shH2afv 24-bp target sequence, is sensitive to shH2afv, but not shH2afz. Similarly, Myc-H2A.Z.2-IS is sensitive to shH2afz, but not shH2afv (Fig. 1G,H, right panels). Taken together, we show that shH2afz and shH2afv are specific for H2afz and H2afv mRNAs respectively, and that we can efficiently knockdown each targeted hypervariant independently.

\section{H2A.Z hypervariant depletion alters expression of nonoverlapping sets of genes}

To examine the impact of individual H2A.Z hypervariant knockdown on basal level of neuronal gene transcription, we performed a genome-wide microarray analysis of RNA obtained from dissociated cortical neurons depleted of H2A.Z.1 or H2A.Z.2. Gene expression levels in these samples were compared to samples from neurons treated with a scrambled shRNA (referred to as control neurons hereafter). The degree of change in the mRNA levels for every gene, whose transcription was altered due to H2A.Z.1 or H2A.Z.2 deficiency (>1.25-fold), is plotted in Figure 2 as a heat map. A total of 338 genes were differentially expressed due to depletion of H2A.Z.1 (154 genes were upregulated and 184 were downregulated), while H2A.Z.2 depletion altered expression of 271 genes (94 genes were upregulated and 177 were downregulated). Only 24 genes were sensitive to depletion of either H2A.Z isoform (Fig. 2B,C; Tables 2-5). Such relative dearth of common genes affected by depletion of either hypervariant suggests that H2A.Z.1 and H2A.Z.2 regulate, directly or indirectly, independent gene cohorts. Functional gene ontology analysis of H2A.Z.1 and H2A.Z.2 target genes revealed that targets of the former are enriched for ionotropic postsynaptic receptors (Fig. 2D). Three of the top 10 molecular GO pathways belonged to this category. 
A

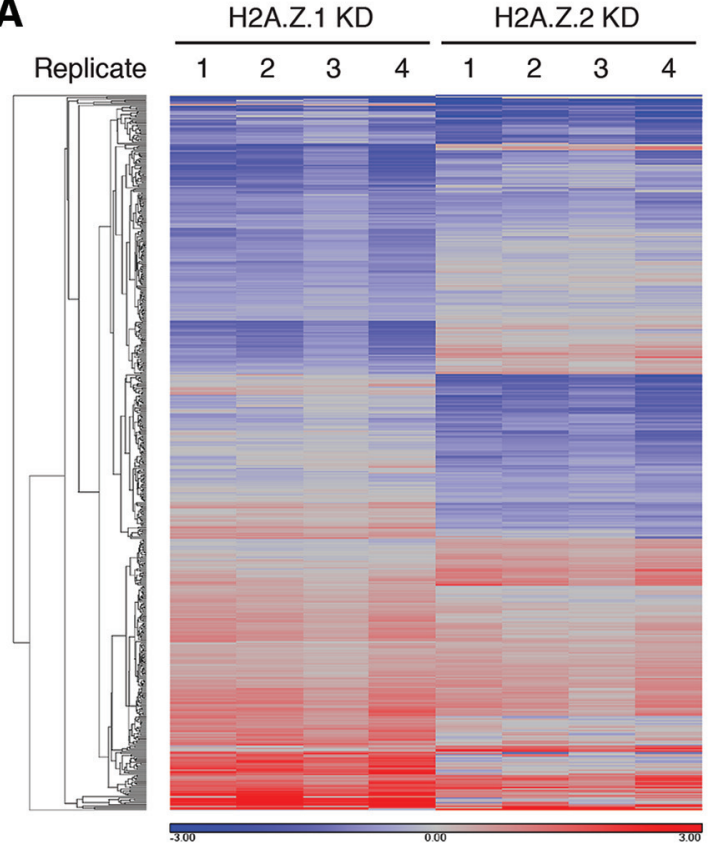

B

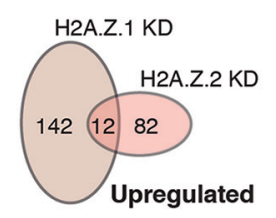

C

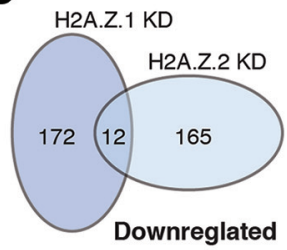

D

\begin{tabular}{|c|c|c|}
\hline & Molecular function GO term & p-value \\
\hline \multirow{5}{*}{ 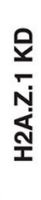 } & Ubiquitin protein ligase binding & 4.25E-04 \\
\hline & Protein binding & 0.002 \\
\hline & Protein kinase A catalytic subunit binding & 0.002 \\
\hline & Ionotropic glutamate receptor activity & 0.002 \\
\hline & $\begin{array}{l}\text { Extracellular-glutamate-gated ion channel } \\
\text { activity }\end{array}$ & 0.003 \\
\hline \multirow{5}{*}{ 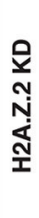 } & Protein binding & 2.56E-05 \\
\hline & $\begin{array}{l}\text { Phosphatidylinositol-4,5-biphosphate } \\
\text { 4-phosphate activity }\end{array}$ & 0.0 \\
\hline & Protein domain specific binding & 0.023 \\
\hline & $\begin{array}{l}\text { Rho guanyl-nucleotide exchange factor } \\
\text { activity }\end{array}$ & 0.026 \\
\hline & GTPase activator activity & 0.038 \\
\hline
\end{tabular}

Figure 2. H2A.Z hypervariant depletion alters baseline expression of mostly nonidentical gene cohorts. $\boldsymbol{A}$, Heat map showing changes in gene expression as detected by microarray analysis after depletion of H2A.Z.1 and H2A.Z.2 compared to control neurons. $N=4$. Genes showing $>1.25$-fold change with $p<0.001$ are shown here. $\boldsymbol{B}, \boldsymbol{C}$, Venn diagrams depicting total number of genes that are either upregulated $(\boldsymbol{B})$ or downregulated $(\boldsymbol{C})$ after depletion of H2A.Z.1 or H2A.Z.2. Note the nonoverlapping nature of such gene sets. $\boldsymbol{D}$, Top five GO terms derived from molecular function gene ontology analysis (DAVID) for H2A.Z.1 and H2A.Z.2 target genes.

This finding contrasts with H2A.Z.2 target genes, where none of the hits were related to synaptic function. Interestingly, knockdown of H2A.Z.1, but not H2A.Z.2, led to altered expression of several candidate genes linked to psychiatric disorders such as autism and schizophrenia (Neurexin1, Shank3, CaMK2G, Vamp7, Gsk3b, Tuba8, Grm8, etc.).

\section{H2A.Z hypervariant depletion changes synaptic protein expression}

Because glutamatergic synaptic function was indicated as a hypervariant-specific GO term in the above analysis, and to test whether H2A.Z hypervariant depletions have any impact on the physiologic health of neurons, we performed electrophysiological analyses of membrane and excitatory synaptic properties. Resting membrane potentials and input resistances were not significantly different between control and H2A.Z.1- or H2A.Z.2-depleted neurons. Presynaptic function and/or synaptic density, as inferred by mEPSC frequency, was also not significantly different between conditions (mEPSC frequency per $3 \mathrm{~s}$ for control, H2A.Z.1-, and H2A.Z.2depleted neurons were $0.31 \pm 0.31,0.14 \pm 0.06$, and $0.13 \pm 0.07$, respectively). However, mEPSC amplitude, typically indicative of postsynaptic function, was significantly diminished in neurons depleted of either H2A.Z hypervariant (Fig. $3 A, B$ ). To better understand mechanisms of such mEPSC amplitude reduction in H2A.Z hypervariant-depleted neurons, we assessed mRNA and protein levels of several postsynaptic molecules, including many from our microarray screen. A few interesting cases representing diverse effects of hypervariant depletion are presented in Figure 3C,D.

A contrasting effect of hypervariant depletion was noted in two instances: postsynaptic density protein 95 (PSD-95) and SHANK3. PSD-95 is the anchoring protein encoded by Dlg4 and found exclusively in the postsynaptic density, while SHANK3 is another member of the postsynaptic density that is encoded by the high-confidence autism candidate gene Shank3. These two gene products were strongly reduced in neurons depleted of H2A.Z.1, but not H2A.Z.2 (Fig. 3D). Compared to control, PSD-95 band intensities were $48.58 \pm 12.4 \%, 102.34 \pm 26.72 \%$, and $65.95 \pm 17.42 \%$ in H2A.Z.1, H2A.Z.2, and both KD, respectively $(N=3)$. Such an effect of H2A.Z.1 KD on PSD-95 must be indirect (not regulated at the gene transcription level), as D/g4 mRNA levels remained unchanged in H2A.Z.1-depleted neurons (Fig. 3C). For SHANK3, compared to control, band intensities were $20.25 \pm 7.6 \%, 47.74 \pm 20.35 \%$, and $15.34 \pm 12.6 \%$ in H2A.Z.1, H2A.Z.2, and both KD, respectively $(N=3)$. However, the effect on SHANK3 is interesting, as Shank3 mRNA levels are upregulated specifically in response to H2A.Z.1 depletion, perhaps as a response to cellular feedback pathways triggered by loss of the protein.

We also tested levels of the ligand-gated ion channel GluA2 (AMPA receptor; coded by Gria2, also called GluR2) and postsynaptic density protein HOMER1 (coded by Homer1) after H2A.Z hypervariant depletion. As shown in Figure $3 D$, we observe no notable difference in signal of either GluA2 or HOMER1B/C after H2A.Z.1 or H2A.Z.2 knockdown. However, the activity-induced short Homer1a 
A
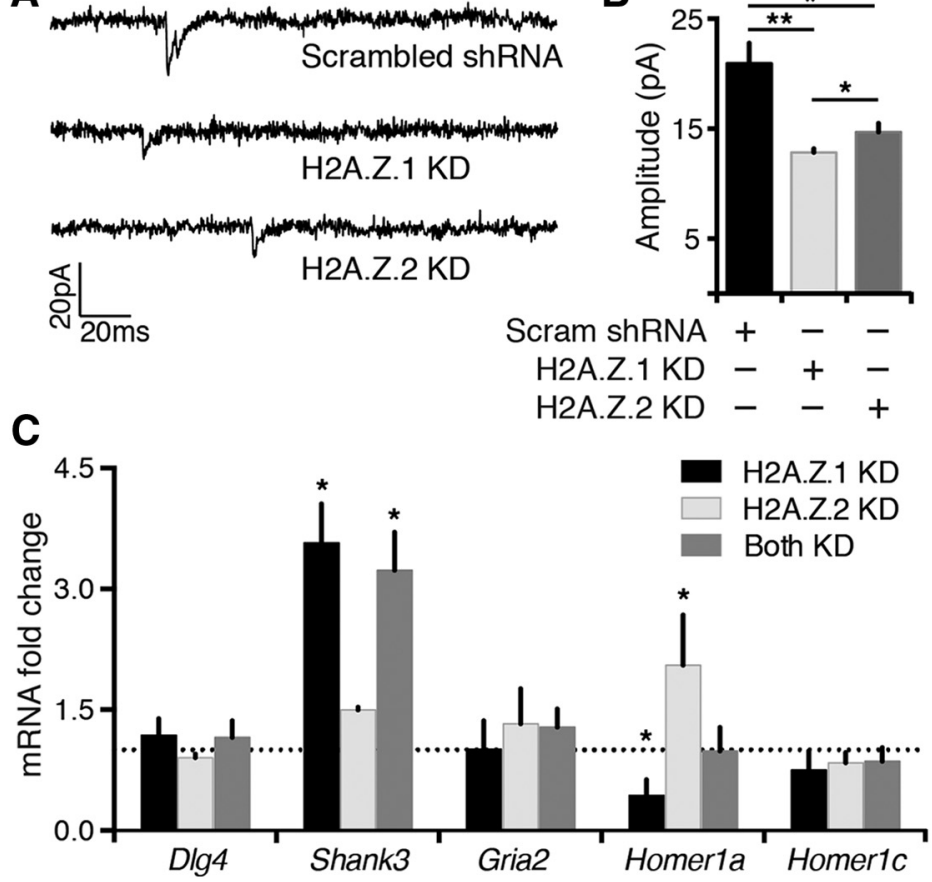

B

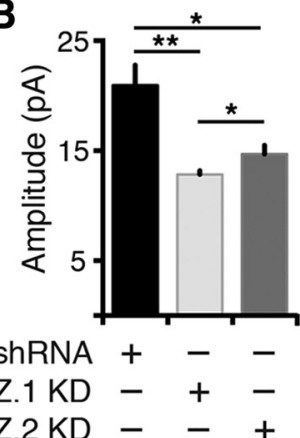

D

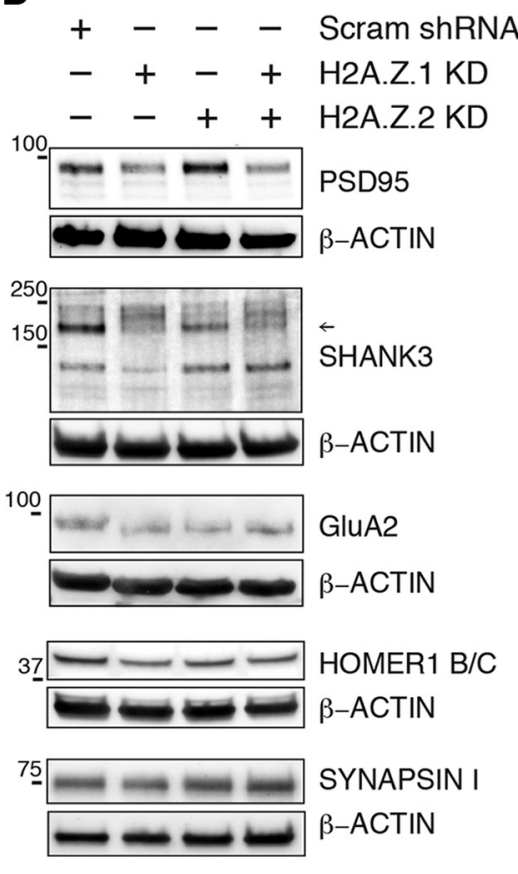

Figure 3. H2A.Z hypervariant-specific RNAi has synaptic effects. A, B, Amplitude of mEPSCs decrease after H2A.Z.1 or H2A.Z.2 depletion. Shown are example traces in $\boldsymbol{A}$ with quantification of mEPSC amplitude is in $\boldsymbol{B}(N=3 ; n=15$ neurons for Sc, 10 for $\mathrm{H} 2 \mathrm{~A}$.Z. 1 $\mathrm{KD}$, and 8 for H2A.Z.2 KD; $* p<0.05$ and $* * p<0.001)$. Neurons were infected for $5-7 \mathrm{~d}$. $\boldsymbol{C}$, mRNA levels of indicated genes, normalized to Gapdh, represented as fold change of their abundance in control neurons (broken line at 1 on $y$-axis). $N=3-5$. $* p<$ 0.05. $\boldsymbol{D}$, Representative Western blottings of synaptic fraction from control or H2A.Z hypervariant-depleted neurons as indicated. Loading control ( $\beta$-ACTIN) for individual blots are provided and molecular weights of the nearest protein ladder band is indicated. Arrow indicates quantified SHANK3 band (see text). Neurons were infected for $7 \mathrm{~d}$. $N=3$. Note: levels of synapsin, a presynaptic molecule, remain unaltered after hypervariant depletions.

mRNA isoform was downregulated by H2A.Z.1 loss and upregulated by H2A.Z.2 depletion (Fig. 3C). Further studies are required to confirm if such effects are direct influences of H2A.Z hypervariants on Homer1a transcription.

Together, we find that depletion of both H2A.Z.1 and H2A.Z.2 leads to decreased amplitude of mEPSCs, which may be explained by altered expression of postsynaptic proteins. The effects of H2A.Z.1 and H2A.Z.2 knockdown on the tested synaptic proteins, however, are apparently mediated by transcription-independent means in most cases given the lack of change at the pre-mRNA level.

\section{H2A.Z.1 and H2A.Z.2 depletion differentially impact Arc/Arg3.1 transcription}

To study direct roles of H2A.Z hypervariants on transcriptional regulation of a model gene, we chose to study the IEG Arc/Arg3.1 (referred to as Arc from here on), which responds rapidly to neuronal activity (Guzowski et al., 1999; Saha et al., 2011). Arc was chosen for several reasons: (1) it is a neuron-specific IEG, which has been extensively studied previously by us and others, (2) its promoter and transcriptional start site (TSS) regions are enriched with H2A.Zcontaining nucleosomes (our unpublished observations), and (3) it is one of the genes recently shown to be regulated by H2A.Z in cognitive functions (Zovkic et al., 2014). Neuronal activity was stimulated in dissociated neurons by $\mathrm{GABA}_{\mathrm{A}}$ receptor antagonist (Bic)-induced disinhibition and $\mathrm{K}^{+}$channel blocker (4AP)-induced increased burst frequency (Bic +
4AP; Papadia et al., 2005). Using the Bic + 4AP protocol, we induced gene transcription and evaluated Arc mRNA abundance at $15 \mathrm{~min}$ after treatment. Basal and induced Arc mRNA levels were unaffected by H2A.Z.1 depletion but were significantly diminished in H2A.Z.2-depleted cells (Fig. 4A).

Because mRNA abundance is not necessarily a reflection of transcription per se but rather the net result of both synthesis and decay rates, we next evaluated the direct Arc transcription product: its pre-mRNA (Wu and Brewer, 2012). We detected no significant differences in the basal level of Arc pre-mRNA with H2A.Z hypervariant depletions (Fig. 4B, 0-min time point). After Bic + 4AP treatment, Arc pre-mRNA levels increased significantly within $15 \mathrm{~min}$ and continued to increase with time in control neurons. However, in agreement with the mRNA data (Fig. 4A), the pre-mRNA response in H2A.Z.2-depleted neurons was attenuated at the early time point $(15 \mathrm{~min})$ and was also significantly reduced at later points (Fig. $4 B$ ). In contrast, H2A.Z.1 depletion had no effect on Arc pre-mRNA levels at $15 \mathrm{~min}$. However, at later time points, H2A.Z.1depleted neurons had significantly less Arc pre-mRNA than in control neurons and yet had significantly more than in neurons depleted of H2A.Z.2 (Fig. 4B). These results suggest that although the loss of H2A.Z.1 impaired Arc transcription significantly at later time points, loss of H2A.Z.2 had a much stronger effect at all time points. At the protein level, hypervariant-specific effects on activityinduced ARC abundance were generally reflective of ArC 
A

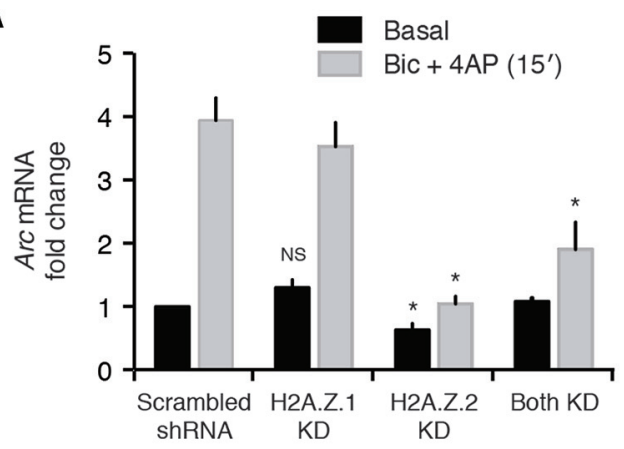

B

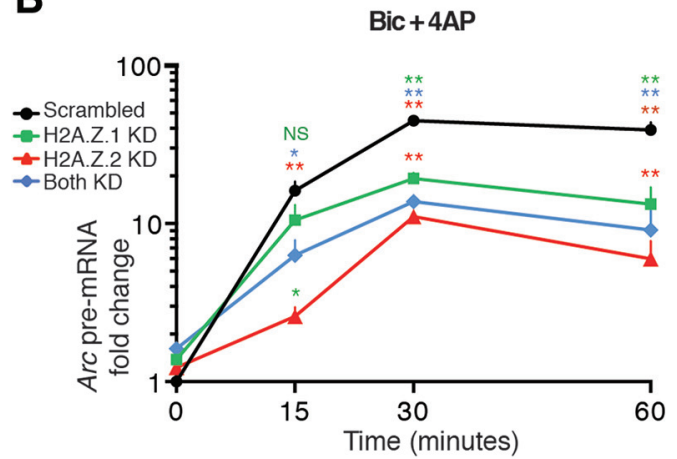

C

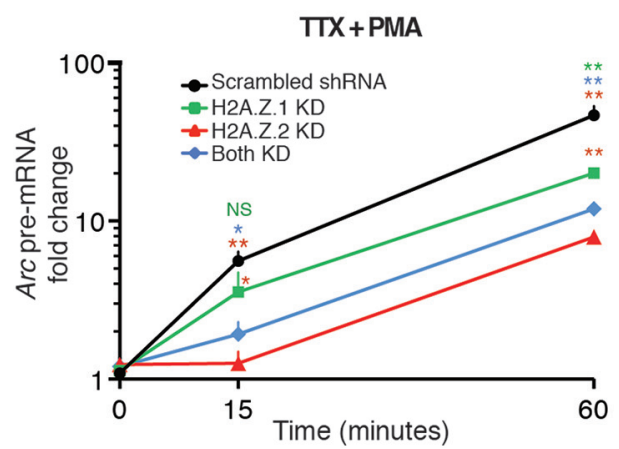

D

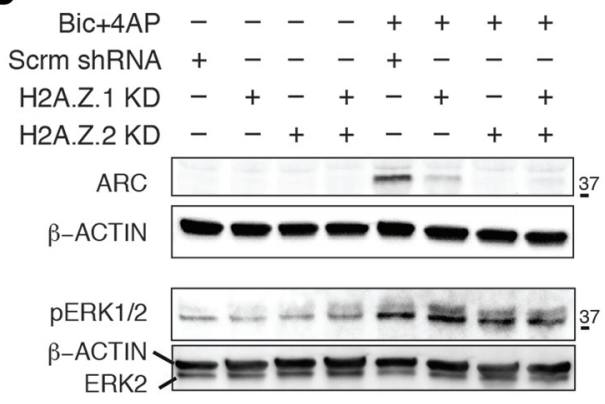

E

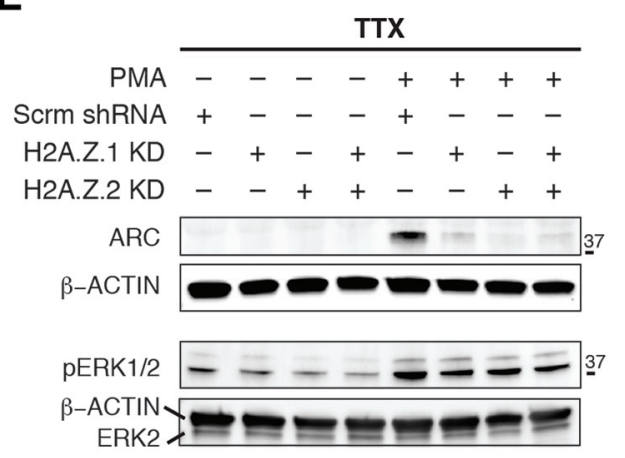

F

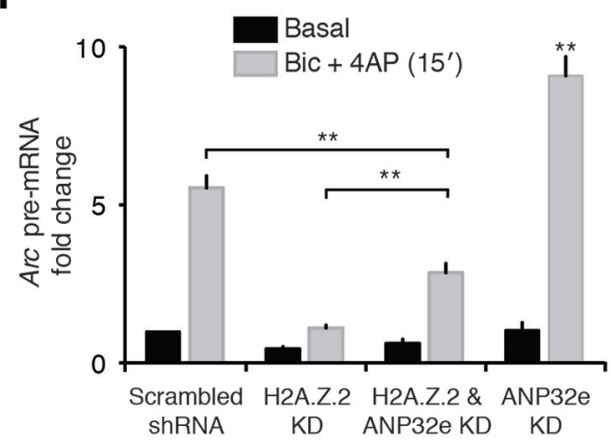

Figure 4. H2A.Z hypervariant-specific RNAi has differential effects on activity-induced Arc transcription. A, Graphical representation of Arc mRNA level at 15 min after treatment with Bic $+4 A P$ in indicated neuronal groups as detected by qPCR and normalized to Gapdh. $N=$ 3-4 for each group; $* p<0.05$. NS, not significant. $\boldsymbol{B}, \boldsymbol{C}$, Representation of Arc pre-mRNA level at different time points after treatment with $\mathrm{Bic}+4 \mathrm{AP}(\boldsymbol{B})$ or TTX + PMA $(\boldsymbol{C})$ in indicated groups of neurons (5-6 d postinfection) as detected by qPCR and normalized to Gapdh. $N$ $=3-4$ for each time point; $* p<0.05$ and $* * p<0.01$. NS, not significant. Two sets of data in statistical comparison are represented by the position and the color of asterisk(s) or a NS. For example, in $\boldsymbol{B}$, the green $*$ on top of the 15 min H2A.Z.2 KD time point (red triangle) represents a statistical difference between the means of H2A.Z.2 and H2A.Z.1 KD group (green square) at the same time point. $\boldsymbol{D}$, $\boldsymbol{E}$, Representative Western blottings of whole-cell lysate from neurons infected as indicated for 5-6 d. Neurons were treated with Bic $+4 \mathrm{AP}$ $(\boldsymbol{D})$ or TTX + PMA $(\boldsymbol{E})$ for either $60 \mathrm{~min}$ to detect ARC, or for $15 \mathrm{~min}$ to detect phosphorylated ERK1/2. $\boldsymbol{F}$, Representation of Arc pre-mRNA level at $15 \mathrm{~min}$ after treatment with Bic $+4 \mathrm{AP}$ in indicated neuronal groups as detected by qPCR and normalized to Gapdh. $N=3 ; * * p<0.01$.

pre-mRNA expression patterns (Fig. 4D). When both hypervariants were depleted, pre-mRNA results were not significantly greater than H2A.Z.2 depletion alone, and were in fact intermediate between the two hypervariants. While trying to knockdown both H2A.Z.1 and H2A.Z.2, we had to titer our viral infection and infect with less of each virus kind to avoid causing viral overload-induced cell death. Therefore, less knockdown was achieved in the dual KD cells compared to their single KD counterparts. Such reduced knockdown may explain the intermediate effect of knocking down both H2A.Z.1 and H2A.Z.2 on Arc transcription.
Because some synaptic alterations were noted due to H2A.Z hypervariant depletion and because Bic $+4 \mathrm{AP}$ treatment is a synapse-dependent activity induction protocol, we next tried to induce Arc nonsynaptically by jump-starting the MAPK pathway, otherwise employed by activity-induced gene transcription signals (Rosen et al., 1994; Wiegert and Bading, 2011). The MAPK pathway was extrasynaptically activated via PKC using PMA (Schultz et al., 1997) in the presence of TTX to prevent any extracellular input via neuronal activity, and this treatment induced rapid transcription of $\operatorname{Arc}$ (Fig. $4 C$ ). When H2A.Z hypervariant-depleted neurons were stimulated using this 
A
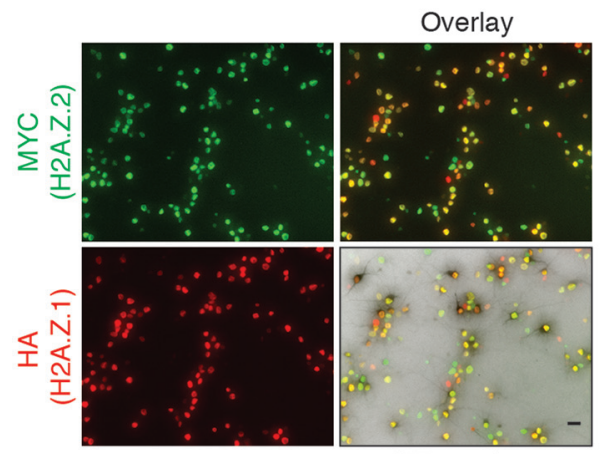

BF overlay
B

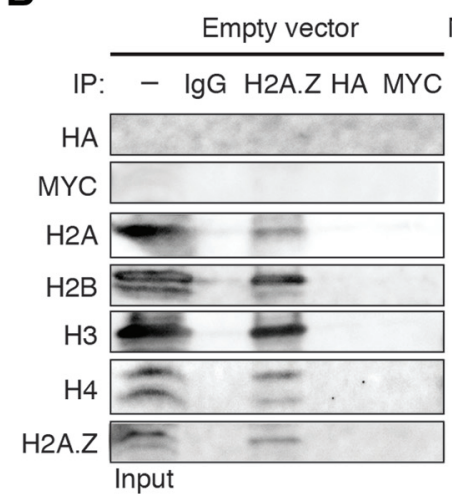

HA-H2A Z 1 Myc-H2A.Z.2

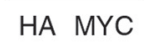

Figure 5. Distinct properties of HA-H2A.Z.1- and MYC-H2A.Z.2-containing nucleosomes. $\boldsymbol{A}$, Representative image of immunocytochemical analysis of HA-H2A.Z.1 and MYC-H2A.Z.2 expression in unstimulated neurons. Approximately $70 \%$ neurons expressed both hypervariants. Scale bar, $20 \mu \mathrm{m}$. B, Co-IP assay from nuclear lysate preparation of neurons exogenously expressing a control vector or HA-H2A.Z.1 and MYC-H2A.Z.2. $N=3$. C, Representative image of genomic DNA obtained from untreated or MNase-treated nuclei of neurons treated as described above. The band size of $\sim 150 \mathrm{bp}$ indicates that MNase digestion predominantly produced mononucleosomes. $N=3$. $D$, Co-IP assay from single nucleosome preparation of neurons exogenously expressing HA-H2A.Z.1 and MYC-H2A.Z.2. Antigen-antibody complex was resolved by electrophoresis and blotted for indicated histones. The 15-kDa protein ladder band is indicated in all panels. Arrow indicates unique band found only in anti-MYC precipitate. $N=3$.

protocol, the outcome was similar to what was seen with the Bic + 4AP protocol, both at the pre-mRNA and protein levels (Fig. 4C,E). These observations suggest that consequences of H2A.Z hypervariant depletion on activity-induced Arc transcription are most likely not limited to effects of changing synaptic components. Supporting this assertion is the observation that H2A.Z hypervariant depletion had no effect on activity-induced activation of the MAPK pathway as indicated by phosphoERK1/2 levels (Fig. 4D,E).

To further test the specificity of the H2A.Z.2 depletioninduced phenotype, we attempted to rescue rapid Arc induction by combining shRNA-mediated knockdown with coexpression of shRNA-insensitive H2A.Z.2 (Myc-H2A.Z.2IS). Several such experimental designs were unsuccessful, likely due to lack of regulatory post-translational modifications on exogenous constructs in the absence of endogenous proteins and/or perturbation of a critical balance of endogenous levels of these hypervariants and their chaperones. The latter possibility is based on a recent study where it was demonstrated that phenotype loss due to depletion of ANP32E, an H2A.Z chaperone that removes nucleosomal H2A.Z, could be rescued by codepletion of ANP32E and H2A.Z (Alatwi and Downs, 2015). Accordingly, we codepleted ANP32E (92.0 $\pm 0.1 \%$ knockdown on day 5 after infection) with H2A.Z.2 and observed a partial rescue of rapid Arc transcription (Fig. $4 F)$. Taken together, our data show nonredundant roles of H2A.Z hypervariants in the regulation of Arc transcription and hint at the importance of a fine balance between histones and their chaperones in mediating such responses.

\section{H2A.Z hypervariants are present near the Arc TSS and regulate its transcription}

H2A.Z is enriched near Arc TSS (Zovkic et al., 2014). To identify specific hypervariant(s) in this enrichment, we exogenously expressed distinctly tagged hypervariants
(HA-H2A.Z.1 and MYC-H2A.Z.2) in neurons and probed for their expression using antibodies against the HA- or MYC-epitope tags (Fig. 5A). Usually, protein overexpression is concerning, because it may cause, among other things, artifacts that include ectopic subcellular localizations and inaccurate protein complex formations. Despite such limitations, we reasoned that increased dosage of tagged-H2A.Z hypervariants in the free-floating nucleoplasmic pool may not have any negative consequences as H2A.Z incorporation into its functionally relevant complex (nucleosomes) is tightly regulated by chaperone-dependent mechanisms (Billon and Cote, 2013), and so this overexpression will not necessarily have unwanted nucleosomal incorporation of H2A.Z despite its artificial abundance.

To verify nucleosomal incorporation of tagged-H2A.Z hypervariants, we performed co-IP assays. These assays, with anti-IgG, anti-H2A.Z, anti-HA and anti-MYC from nuclear lysates, or anti-HA and anti-MYC from mononucleosome preparations (Fig. 5B-D), detected stable association of both hypervariants with other nucleosomal histones $\mathrm{H} 2 \mathrm{~B}, \mathrm{H} 2 \mathrm{~A}, \mathrm{H} 3$ and $\mathrm{H} 4$ suggesting that taggedH2A.Z molecules are incorporated into nucleosomes. Interestingly, (1) HA and MYC IPs probed for their respective hypervariants revealed very little association between HA-H2A.Z.1 and MYC-H2A.Z.2, suggesting that very few H2A.Z nucleosomes contain both hypervariants; and (2) we detected a unique band in $\mathrm{H} 2 \mathrm{~B}$, presumably representing an unidentified post-translationally modified state of $\mathrm{H} 2 \mathrm{~B}$, in the precipitate with MYC-H2A.Z.2, but not HA-H2A.Z.1 (Fig. 5B,D). These data suggest that certain H2A.Z.2-containing nucleosomes may have unique posttranslational signatures and possibly unique functions.

To confirm that functional incorporation of taggedH2A.Z molecules into the Arc promoter region did not interfere with transcriptional activity, we expressed HAH2A.Z.1 and MYC-H2A.Z.2 in mature neurons and induced Arc transcription with PMA + TTX. Overexpression 
A

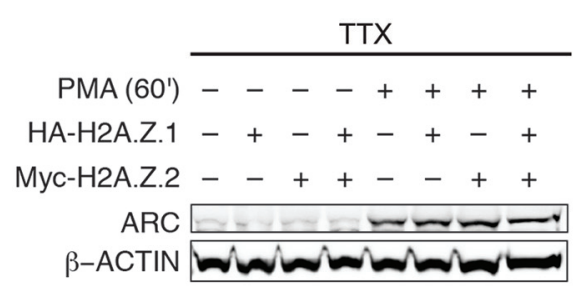

C
B

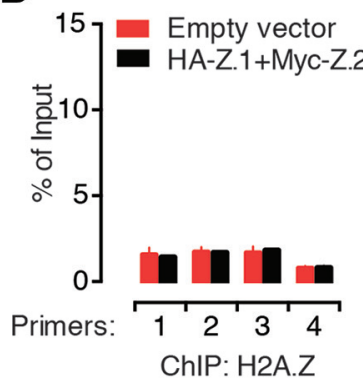

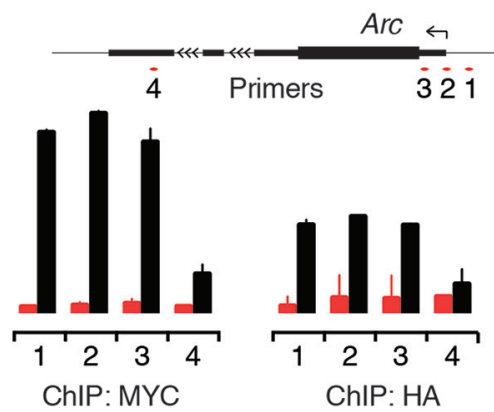
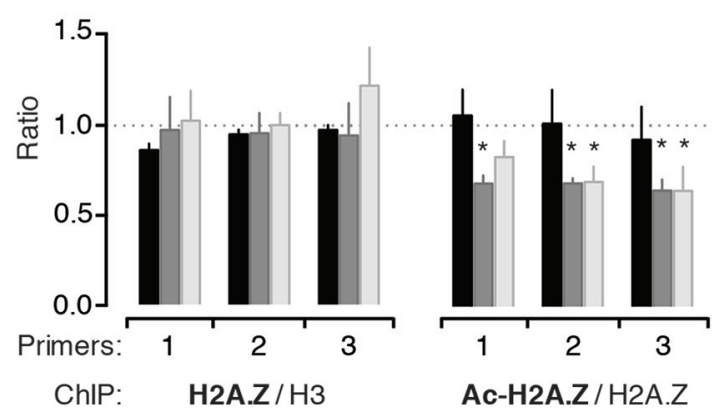

Ac-H2A.Z/H2A.Z

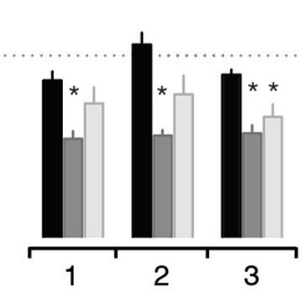

MYC / H2A.Z
Bic + 4AP (5 min) $\mathrm{Bic}+4 \mathrm{AP}(15 \mathrm{~min})$ $\mathrm{Bic}+4 \mathrm{AP}(30 \mathrm{~min})$

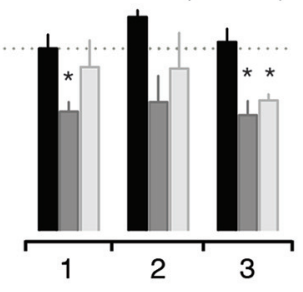

HA / H2A.Z

Figure 6. Both $\mathrm{H} 2 \mathrm{~A} . \mathrm{Z}$ hypervariants are present near the Arc TSS and mediate its transcription. $\boldsymbol{A}$, Representative Western blotting of whole-cell lysate from neurons infected as indicated for 5-6 d. Neurons were treated with TTX + PMA for 60 min to assess ARC expression. $N=3$. B, top, Graphical map (not to scale) depicting relative position of ChIP primers near Arc TSS. Bottom, Quantification of H2A.Z, MYC-H2A.Z.2, and HA-H2A.Z.1 binding to the Arc TSS region in untreated neurons as determined by ChIP with antibodies against H2A.Z, MYC, and HA epitopes. N = 4. C, Quantification of H2A.Z, Ac-H2A.Z, MYC-H2A.Z.2, and HA-H2A.Z.1 binding to Arc TSS region in neurons treated with Bic +4 AP for indicated time periods. $N=4$. Data are normalized as indicated and expressed as fold change compared to untreated samples (denoted by the broken line at 1 on $y$-axis).

of one or both hypervariants did not alter Arc transcription at pre-mRNA or protein level (Fig. 6A).

Next, we performed ChIP assays to detect H2A.Z and its hypervariants near the Arc TSS. We tested several anti-H2A.Z antibodies and found the ChIP grade antibody from Millipore to be most efficient. Using this antibody, we detected significant enrichment of H2A.Z near the Arc TSS (primer pair 1, 2, and 3) compared to Arc gene body (primer 4; Fig. 6B). Primer pairs 1 and 3 roughly represent the -1 and +2 (often referred to as +1 ) nucleosomes across the Arc TSS (Kim et al., 2010), while primer pair 2 denotes a region across the TSS, which instead of being nucleosome-free, houses a nucleosome $(+1)$ in $\operatorname{Arc}$ (see H3K4.me data in the study by Kim et al., 2010). Like total H2A.Z, both MYC-H2A.Z.2 and HA-H2A.Z.1 were also enriched near the TSS compared to the gene body (Fig. $6 B$ ). Although strong signals were obtained near the TSS with both anti-HA and anti-MYC ChIP compared to empty vector controls, these signals cannot be directly compared as their apparent difference is likely to be due to dissimilarity in antibody efficiency. For example, this trend is reversed when similar ChIP assays were performed after overexpressing hypervariants with swapped tags (data not shown). Taken together, we conclude that both H2A.Z hypervariants are present near the Arc TSS.

To distinguish the potentially different roles of H2A.Z hypervariants in activity-induced Arc transcription at the gene level, we treated neurons with Bic $+4 A P$ for various time periods and performed ChIP with anti-
H2A.Z, anti-MYC, anti-HA, and anti-acetylated H2A.Z (Ac-H2A.Z) antibodies (Fig. 6C). Acetylation of H2A.Z is an important epigenetic modification associated with active or primed promoters (Soboleva et al., 2014). On Bic + 4AP treatment, H3-normalized total H2A.Z levels were unchanged at any of the three nucleosomes across the Arc TSS at all time points tested (see Discussion). However, ChIP signal for Ac-H2A.Z drops significantly at all three nucleosomes at $15 \mathrm{~min}$ after treatment and remains reduced in the +1 and +2 nucleosomes after $30 \mathrm{~min}$. Although total level of H2A.Z remained unchanged, we detected a significant drop of H2A.Z-normalized MYC-H2A.Z.2 and HA-H2A.Z.1 at the +2 nucleosome 15 and 30 min after treatment, suggesting ongoing activity-induced nucleosomal turnover of H2A.Z (nucleosomal remodeling). Interestingly, at the +1 nucleosome, only MYC-H2A.Z.2 levels dropped significantly at $15 \mathrm{~min}$ after treatment, indicating a possible H2A.Z.2-specific role for this nucleosome at this early time point. This inference is corroborated by our data in that the most severe effect on Arc induction was found after the depletion of H2A.Z.2 (Fig. 4B-E).

\section{H2A.Z.2 is required for RNA Pol II pausing near the Arc TSS}

To study the underlying mechanism of H2A.Z.2's role in rapid Arc transcription (Fig. 4), we tested the effect of its knockdown on histone acetylation and promoter proximal RNA Pol II pausing based on recent reports (Saha et al., 
A

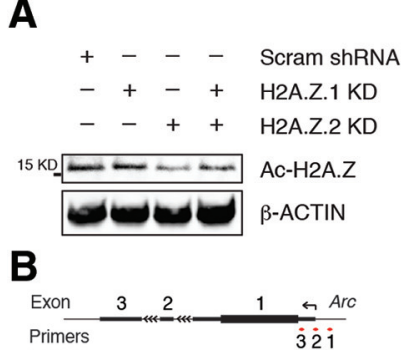

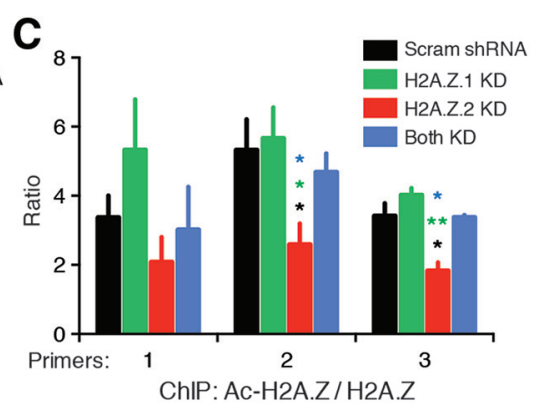

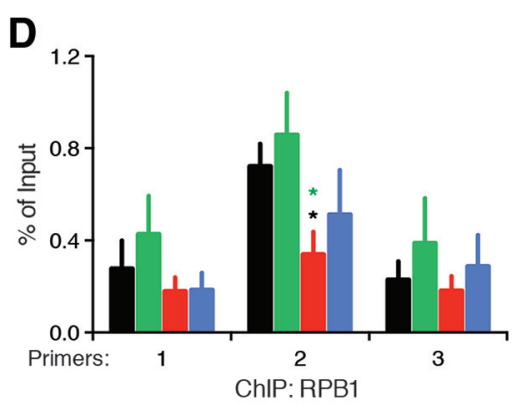

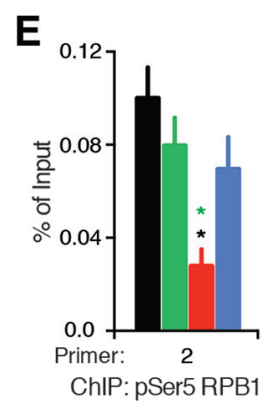

Figure 7. H2A.Z.2 facilitates priming of the Arc promoter. $\boldsymbol{A}$, Representative Western blotting of whole-cell lysate showing Ac-H2A.Z levels in neurons depleted of H2A.Z hypervariants as indicated. $N=3 . B$, Graphical map (not to scale) depicting relative position of ChIP primers near Arc TSS. C, Quantification of Ac-H2A.Z (normalized by total H2A.Z) binding to Arc TSS region in neurons treated as indicated, determined by ChIP. $N=4$. Two sets of data in statistical comparison are represented by the position and the color of asterisk(s). $\boldsymbol{D}$, ChIP data demonstrating binding of RPB1 to the Arc TSS region in control neurons or H2A.Z hypervariant-depleted neurons as indicated. $N=$ 4. Note the enrichment of RPB1 at the TSS (primer 2) in control neurons (black) indicating promoter-proximal RNA Pol II pausing. $\boldsymbol{E}$, ChIP data demonstrating binding of pSer5-RPB1 to the Arc TSS in control and hypervariant-depleted neurons. $N=3$. $* p<0.05$ and $* * p<0.01$.

2011; Vardabasso et al., 2015). In melanoma cells, knockdown of H2A.Z.2, but not H2A.Z.1, attenuates acetylation of histone $\mathrm{H} 3$ and $\mathrm{H} 4$ globally (Vardabasso et al., 2015). We observed no such effect of H2A.Z.2 depletion on H3 or $\mathrm{H} 4$ acetylation in neurons (data not shown). This effect is presumably a cell-type-specific phenomenon relevant to dividing cells. Instead, we noted that H2A.Z.2 depletion led to a decrease in Ac-H2A.Z levels globally (Fig. 7A) and near the Arc TSS (Fig. 7B,C). Note that the data presented in Figure $7 \mathrm{C}$ are normalized by total H2A.Z, the level of which remained unaltered after hypervariant knockdown. The effect was not mimicked by H2A.Z.1 depletion. This suggests that H2A.Z.2 depletion results in selective loss of acetylation, not total H2A.Z, near the TSS.

Presence of TSS proximal H2A.Z highly correlates with another chief component of rapid IEG transcription, RNA Pol II pausing (Day et al., 2016). We have previously shown that RNA Pol II is loaded and paused near TSS of Arc and other rapidly induced IEGs, and underlies the ability of these genes to respond to activity within a few minutes (Saha et al., 2011). Here, we tested whether there is a relation between Pol II pausing and H2A.Z hypervariants at the ArC TSS. Using ChIP with an antibody against RPB1 (N-terminus), the largest Pol II subunit, we found that RNA Pol II pausing at the Arc TSS is diminished significantly in neurons depleted of H2A.Z.2, but not H2A.Z.1. While it is unlikely that our antiRPB1 antibody precipitates nonspecific proteins or is less efficient after hypervariant depletion, we sought to corroborate our findings with another antibody to further ensure that H2A.Z.2 depletion was affecting paused Pol II. We performed ChIP with an antibody specific to RPB1 phosphorylated at serine 5 (pSer5) residue of C-terminal domain heptad repeats. pSer5-RPB1 represents the pool of RPB1 that is transcriptionally engaged, but has not entered productive elongation (paused). Our data indicate that actively engaged Pol II at the +1 nucleosome of $A r c$ is significantly reduced by the loss of H2A.Z.2, but not H2A.Z.1 (Fig. 7E). This hypervariant-specific loss of Pol II pausing provides an explanation for our observations at 15-min post-Bic + 4AP treatment (Fig. 4B,C), where Arc pre-mRNA levels were minimal in H2A.Z.2-, but not in H2A.Z.1-, depleted neurons.

\section{H2A.Z.1 plays context-dependent roles in Arc transcription}

To determine a potential role for H2A.Z.1 in Arc transcription, we hypothesized that it may facilitate Pol II recruitment or elongation during active transcription. To test these possibilities, we induced Arc transcription using the DRB washout protocol with or without Trip. DRB is a nucleoside analog that inhibits mammalian transcription by interfering with the DRB sensitivity-inducing factor complex that enables RNA Pol II pausing in the presence of negative elongation factor (NELF), and then facilitates productive elongation once NELF falls off in response to external signals (Kwak and Lis, 2013). Because DRB can be washed out, DRB washout assays have been used to achieve rapid and synchronized transcription elongation without additional extranuclear signals (Lee et al., 2015). When control neurons were subjected to this assay, we observed a rapid and robust Arc induction (Fig. 8A). DRB washout resulted in significant upregulation of Arc premRNA levels within three minutes, which then continued to increase with time to reach $>100$-fold at $15 \mathrm{~min}$. Depletion of $\mathrm{H} 2 \mathrm{~A} . \mathrm{Z} .2$ resulted in, as we saw with $\mathrm{Bic}+4 \mathrm{AP}$ and TTX + PMA assays, an attenuated response at the early time point that then partially recovered at later time points while remaining significantly less than the normal response (Fig. 8A). In contrast, depletion of H2A.Z.1 had no effect on DRB washout-triggered robust Arc transcription (Fig. $8 A$ ), indicating that H2A.Z.1 is likely not involved in the productive elongation phase.

Next, to test for any role of H2A.Z in RNA Pol II recruitment, a phenomenon that sustains robust responses by providing the gene promoter with a chain of Pol II complexes, we washed out DRB in the presence of Trip, a diterpene triepoxide isolated from natural sources. Trip inhibits Pol II recruitment to promoters by inhibiting TFIIH, the pre-initiation complex component that mediates Pol II binding to DNA (Titov et al., 2011). When DRB washout was performed in the presence of a low concentration of Trip, Arc transcription was significantly reduced at later time points (Fig. $8 A$ ) suggesting that the slightest interference with Pol II recruitment compromises the robustness 
A

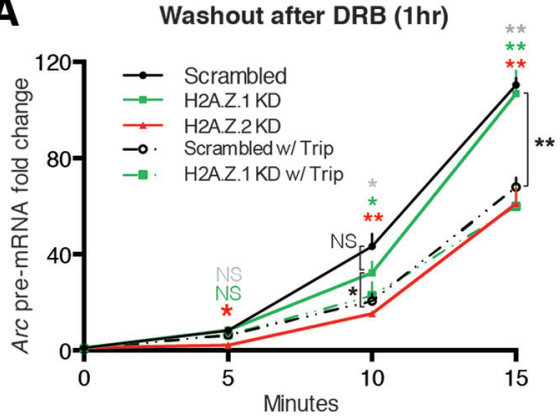

B

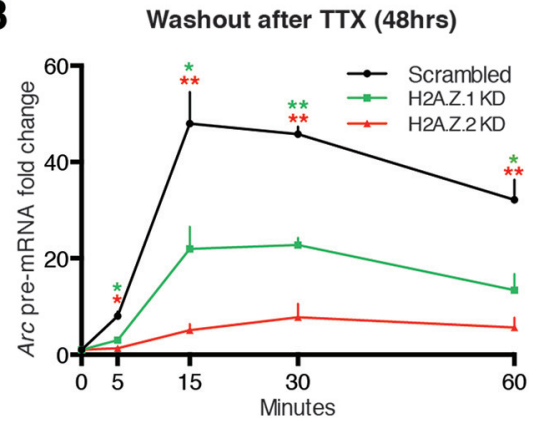

Figure 8. H2A.Z.1 plays context-dependent roles in Arc transcription. A, Graphical representation of the level of Arc pre-mRNA at different time points after 1-h treatment with DRB followed by its washout for indicated time periods in the presence or absence of Trip in indicated groups of neurons (infected for 5-6 d) as detected by qPCR and normalized to Rn18s (18s rRNA; DRB inhibition-insensitive, Pol III-dependent transcription). Gray, green and red asterisk(s) or NS represent statistical comparison between control (Scrambled) responses and responses in Scrambled w/Trip, H2A.Z.1 KD w/Trip and H2A.Z.2 KD, respectively. Connecting brackets delineate other comparisons, shown in black asterisk(s) or NS. B. Similar dataset as in $\boldsymbol{A}$, except that control neurons or hypervariant-depleted neurons were treated with TTX for $48 \mathrm{~h}$ followed by its washout for indicated time periods. Two sets of data in statistical comparison are represented by the position and the color of asterisk(s) or NS. For both $\boldsymbol{A}, \boldsymbol{B}, N=4-5$ for each time point; $* p<0.05$ and $* * p<0.01$. NS, not significant.

of the response. Had H2A.Z.1 played a role in Pol II recruitment, its depletion would have had an effect comparable to washout with Trip in control cells. However, that does not seem to be the case as the Arc response is significantly different between these two groups (Fig. 8A, compare broken black line with green line). Moreover, control and H2A.Z.1-depleted neurons responded similarly to induction in the presence of Trip (Fig. 8A, compare broken black and green lines), suggesting that H2A.Z.1 is unlikely to be required for Pol II recruitment.

To test the roles of H2A.Z hypervariants in another neurobiological context, we next performed the TTX washout assay (Rao et al., 2006; Saha et al., 2011). This assay involves treating neurons with TTX for $48 \mathrm{~h}$ to induce homeostatic synaptic changes, followed by TTX washout to trigger rapid and robust network activity resulting in Arc transcription (Fig. 8B). Consistent with other induction assays so far, H2A.Z.2 depletion had an attenuating effect on early transcriptional response, followed by partial recovery at later time points (Fig. $8 B$ ). Surprisingly, depletion of H2A.Z.1 significantly reduced the ArC response throughout, even the early time points. This is in contrast with the insensitivity of the Arc response at early time points in all induction assays, including 15-min postinduction with Bic $+4 \mathrm{AP}$ under resting (control) conditions. Together, our data suggest that H2A.Z.1 exerts context-dependent effects on activity-induced Arc transcription.

\section{Gene- and context-specific roles of H2A.Z hypervariants in rapid IEG transcription}

In a previous study, we identified two groups of IEGs classified based on the presence or absence of paused Pol II near the promoter (Saha et al., 2011). We refer to these two groups as rapid IEGs (genes with paused Pol II) and delayed IEGs (genes without paused Pol II; Saha and Dudek, 2013). Because H2A.Z.2 likely regulates Arc transcription via RNA Pol II pausing, we next asked if the roles played by these hypervariants are universal across other rapid IEGs. Several rapid IEGs, identified in our previous screen, were then studied under two neurobiological contexts: (1) activity induced in previously untreated neurons (Bic + 4AP assays); and (2) activity induced after homeostatic changes (TTX washout assays). Responses of a few IEGs in control and H2A.Z hypervariant-depleted neurons are shown in Figure 9, and salient inferences drawn from these data are noted in the following paragraph.

All IEGs in Figure 9 respond to activity under both normal and homeostatic conditions. With some genes, such responses are more robust at early time points after $48 \mathrm{~h}$ of TTX treatment (Npas4, cFos, Cyr61, etc.). In general though, most rapid IEGs maintained similar temporal dynamics of expression in both conditions; Btg2 was an exception in that its response peaked at different times under different conditions. In most of these instances, depletion of either H2A.Z.1 or H2A.Z.2 had either no effect or reduced gene transcription. Fbxo33 was an exception to this observation, with H2A.Z.1 depletion resulting in a significantly stronger transcriptional response at the early time point in the Bic + 4AP condition only. Several genes (Cyr61, Btg2, and Dusp1) resembled Arc in that H2A.Z.2 depletion caused the most severe reductions. In contrast to Arc and these genes, H2A.Z.1 or H2A.Z.2 knockdown exerted statistically similar effect on several rapid IEGs (Npas4, cFos, Nr4a3, etc.) under both contexts. Interestingly, we noticed that H2A.Z hypervariant depletions induced different outcomes under different contexts in a few cases. For example, H2A.Z.2 depletion did not alter Btg2 and Dusp1 responses significantly at the early time point under normal conditions, but impaired them at the same time point after $48 \mathrm{~h}$ of TTX. Interestingly, these two genes are insensitive to H2A.Z.1 depletion for both contexts at all tested time points. Collectively, our data in Figure 9 suggest that H2A.Z.1 and H2A.Z.2 do not have generalized roles in rapid IEG transcription; instead, they play genespecific roles, which may vary further under different neurobiological contexts.

To further investigate the role of H2A.Z hypervariants in IEG transcription, we conducted NanoString analyses, a 

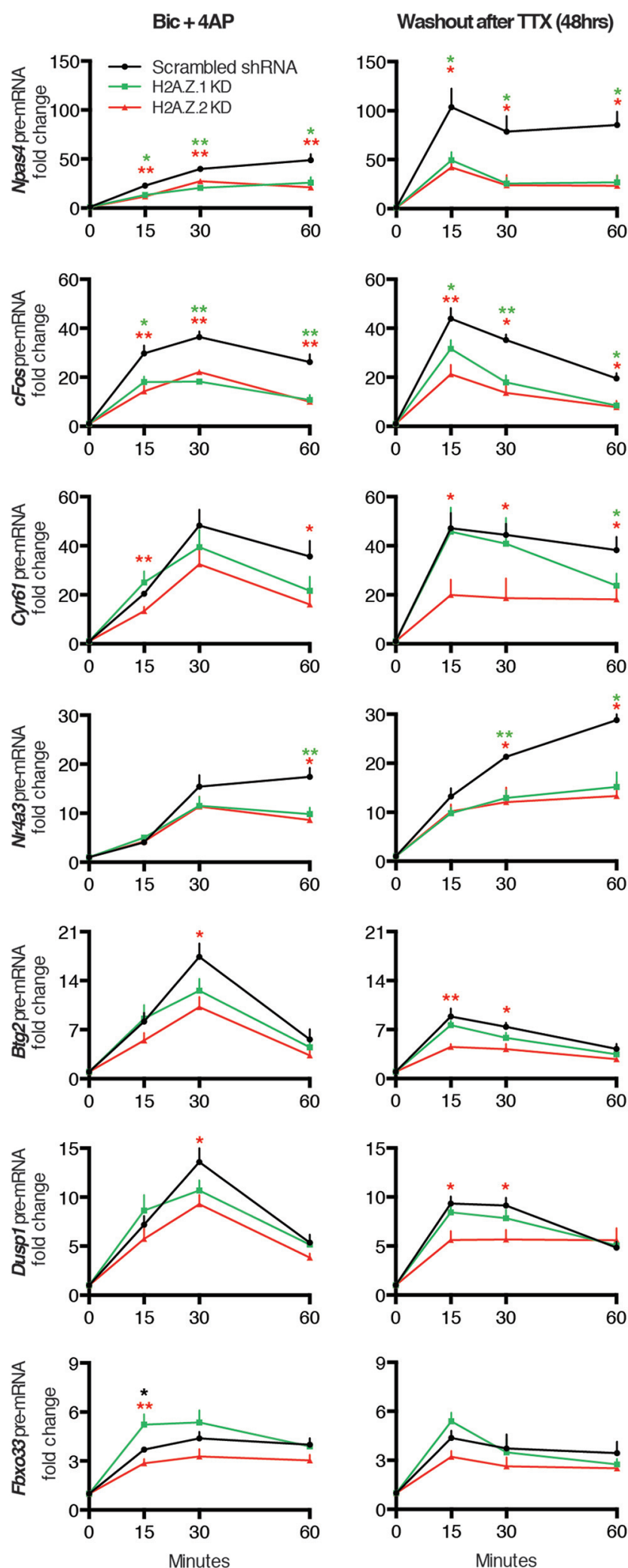

Figure 9. H2A.Z.1 and H2A.Z.2 play context-dependent roles in activity-induced transcription of rapid IEGs. Graphical representation of pre-mRNA levels for noted IEGs at different time points after two treatment regimens. Left, Bic + 4AP treatment. Right, 48-h treatment with TTX followed by its washout for specified time periods in indicated groups of neurons as detected by
Figure 9. continued

qPCR and normalized to Gapdh. $N=4-7$ for each time point; $* p<0.05$ and $* * p<0.01$. NS, not significant. Two sets of data in statistical comparison are represented by the position and the color of asterisk(s).

multiplexed gene expression analysis system (Geiss et al., 2008), on control and hypervariant-depleted cells under the different neurobiological contexts described above using all IEGs identified in our previous screen (Saha et al., 2011). Figure $10 A, B$ shows the results for rapid IEGs and non-IEGs (control genes) displayed as heat maps. Notably, results of the NanoString analyses were consistent with our initial GPCR analyses of rapid IEGs in Figure 9. Although there appeared to be gene-specific regulation due to loss of H2A.Z.1 and/or H2A.Z.2, overall comparison of all rapid IEGs tested in control neurons to those tested in hypervariant-depleted neurons suggests that loss of either hypervariant generally resulted in diminished transcription of rapid IEGs at each time point under both neurobiological contexts (Fig. 10C,D).

\section{Gene- and context-specific roles of H2A.Z hypervariants in transcription of delayed IEGs}

Because we saw gene-to-gene variation in H2A.Z hypervariant regulation of rapid IEG transcription, we asked if these hypervariants might also be involved in regulating transcription of delayed IEGs. Based on the lack of a role for paused Pol II in the delayed IEG transcription, we expected to find no effect of hypervariant depletion. However, we found a context-dependent regulation of delayed IEGs, and some trends are noted along the following lines: at 60-min postinduction, depletion of H2A.Z.1 appears to result in downregulation of Cox2, Klf4, and Nur77 with Bic $+4 \mathrm{AP}$ induction, yet results in upregulation of the same genes on induction after TTX-induced changes. Differential regulation between neurobiological contexts was also evident after loss of H2A.Z.2 in Cartpt and Fam46a (Fig. $11 A)$. Interestingly, group statistical comparisons of delayed IEG responses in hypervariant-depleted neurons to control neurons show that loss of H2A.Z.1 only has a significant effect at the early time point on induction after $48 \mathrm{~h}$ of TTX. However, on induction in normal neurons (Bic $+4 \mathrm{AP}$ assays), H2A.Z.1 depletion only significantly affects the group at the late time point (Fig. 11B). Overall, these data suggest that the roles of H2A.Z hypervariants in regulating transcription of delayed IEGs may vary depending on specific genes and cellular states.

\section{Discussion}

H2A.Z is enriched in nucleosomes near promoters and enhancers of many vertebrate genes and therefore is postulated to play a critical role in gene transcription (Farris et al., 2005; Raisner and Madhani, 2006; Zlatanova and Thakar, 2008). However, roles of the hypervariants H2A.Z.1 and H2A.Z.2 in these processes have often been presumed to be redundant, and have not been categorically questioned (Soboleva et al., 2014). In the current study, we have demonstrated several instances of H2A.Z.1 and H2A.Z.2 nonredundancies: (1) H2A.Z.1 and 
A

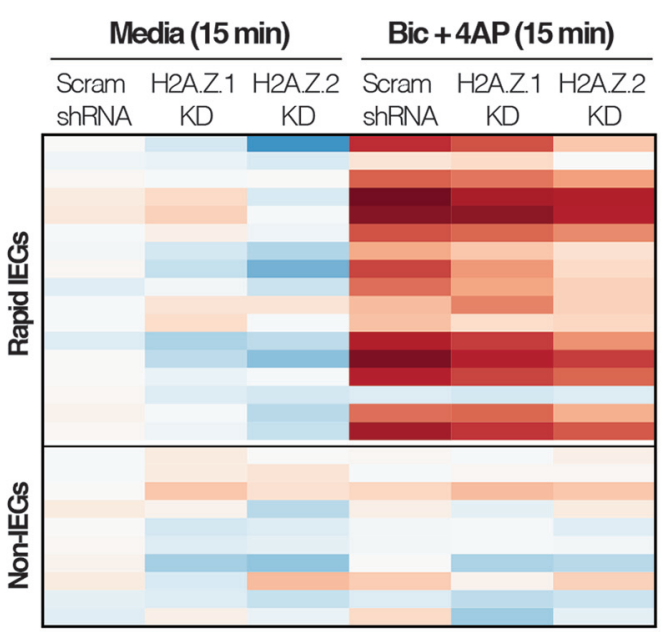

B

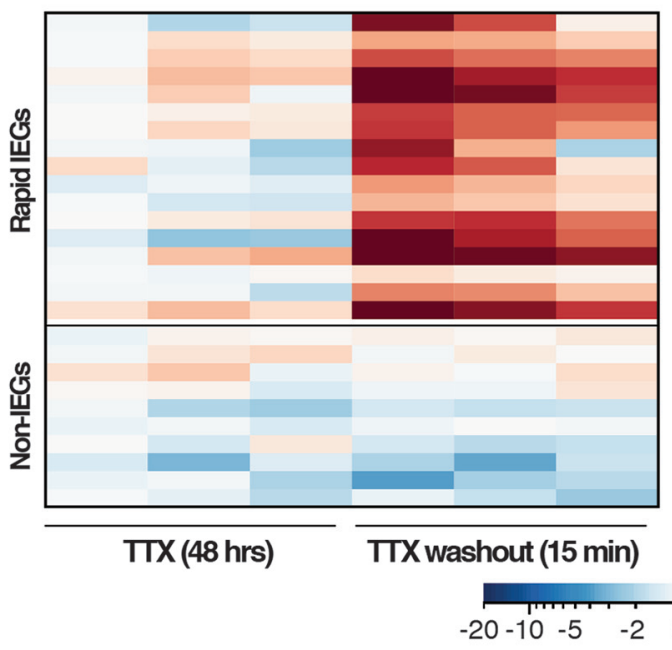

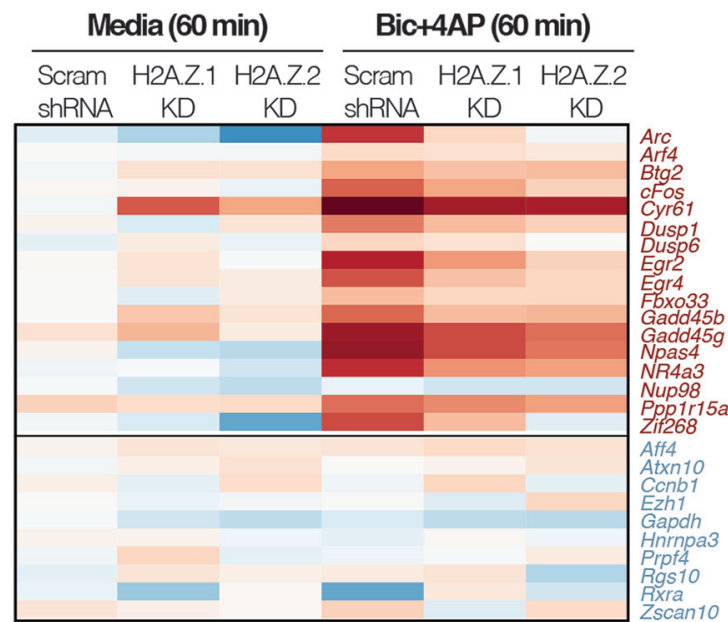

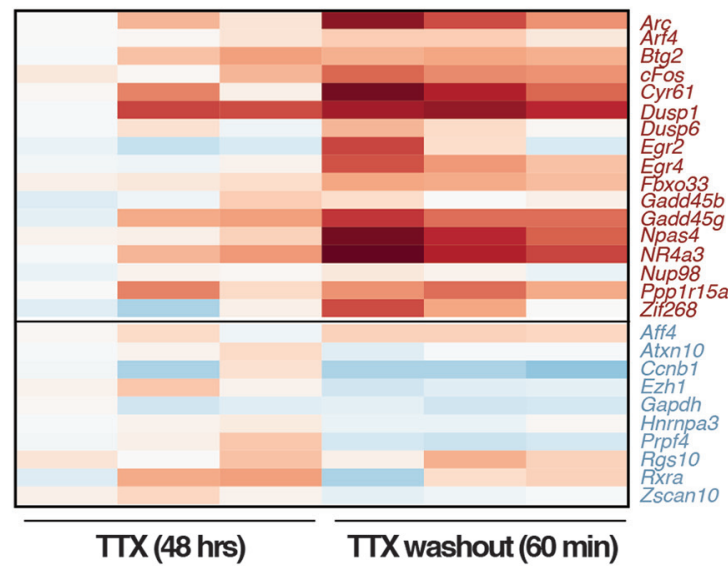

C

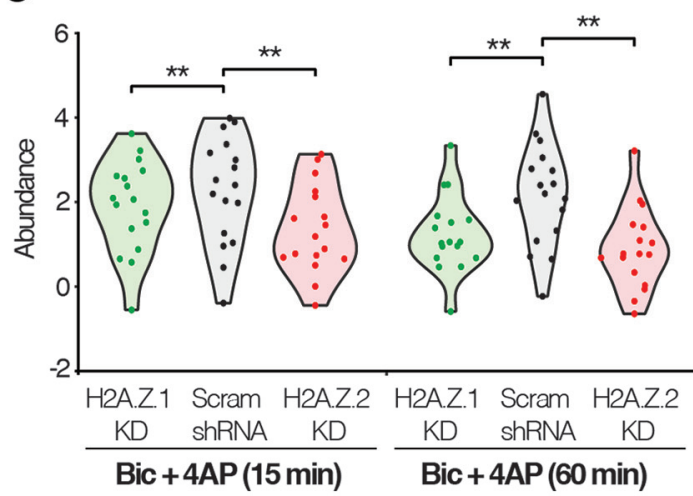

D

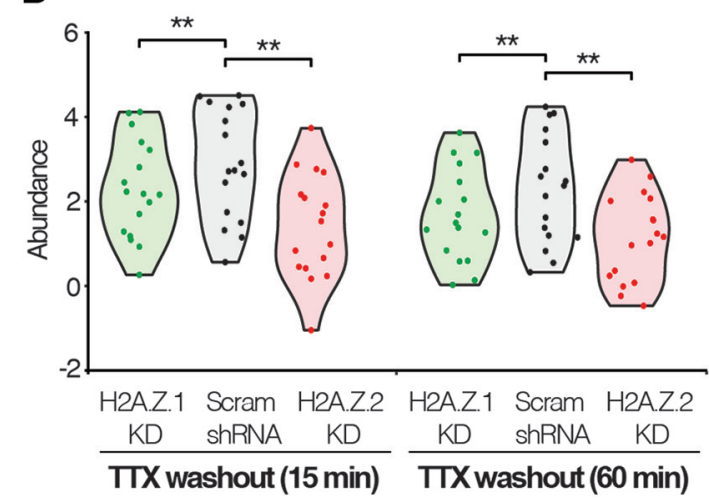

Figure 10. Effect of H2A.Z hypervariant depletion on transcription of rapid IEGs and nonactivity-induced genes. $\boldsymbol{A}$, Nanostring multiplexed gene expression data (heat maps) representing pre-mRNA levels in control and hypervariant-depleted neurons treated with $\mathrm{Bic}+4 \mathrm{AP}$ for indicated times. $\boldsymbol{B}$. Heat maps representing pre-mRNA levels in control and hypervariant-depleted neurons treated TTX for $48 \mathrm{~h}$ followed by washout and collected at indicated times. $\boldsymbol{C}, \boldsymbol{D}$, Violin plots depicting pre-mRNA abundance of all rapid IEGs from hypervariant-depleted neurons compared to that of control neurons treated with Bic $+4 \mathrm{AP}(\boldsymbol{C})$ or TTX for $48 \mathrm{~h}$ followed by washout $(\boldsymbol{D})$ for indicated times. $N=3$ for each treatment all time points. $* p<0.05$ and $* * p<0.01$ (Wilcoxon paired nonparametric test).

H2A.Z.2 depletion in cortical neurons affects basal expression of predominantly nonoverlapping sets of genes; (2) depletion of these hypervariants has differential effects on important synaptic molecules like PSD-95 and SHANK3; (3)
$\mathrm{H} 2 \mathrm{~B}$, perhaps when modified in a certain way, interacts with MYC-H2A.Z.2, but not HA-H2A.Z.1; (4) H2A.Z.1 and H2A.Z.2 depletion differently influence activity-induced Arc transcription kinetics; (5) in normal neurons, H2A.Z.2, 
A

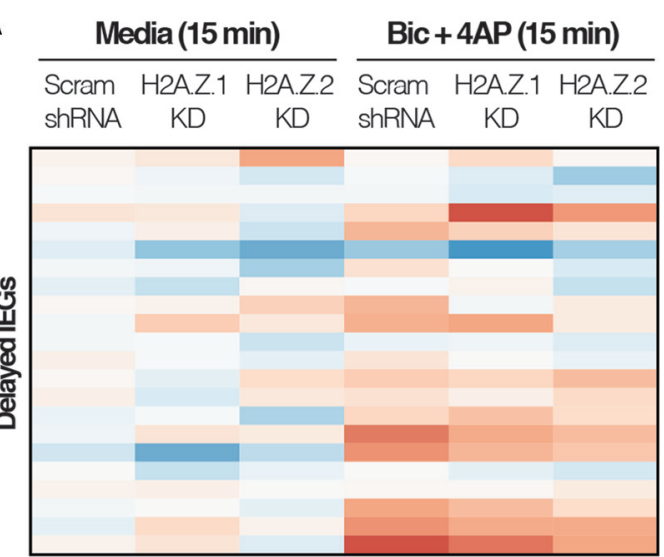

B

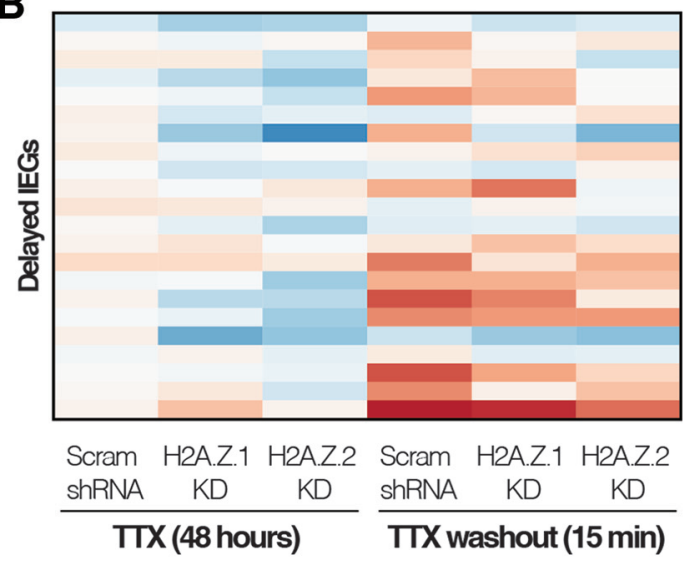

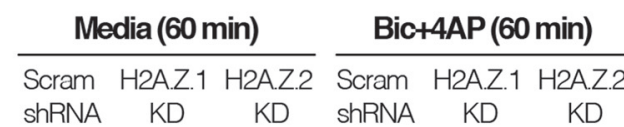
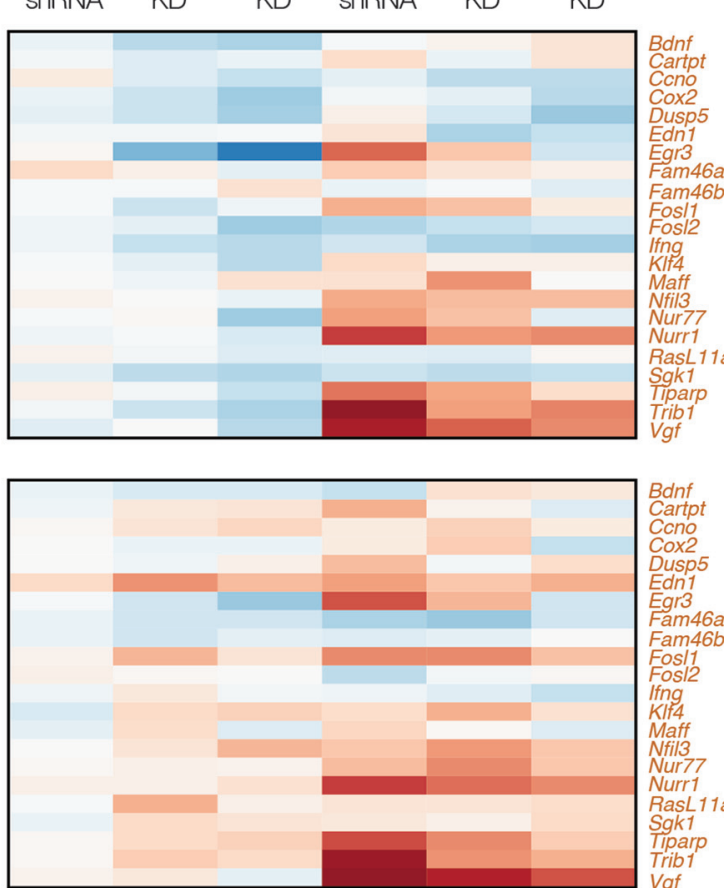
Bdnf
Cartpt
Cono
Cox2
Dusp5
Edn1
Egr3
Fam46a
Fam46b
Fosl1
Fosl2
Ifng
Klit4
Maff
Nfil3
Nur77
Nurr1
RasL11
Sgk1
TIparp
Trib1
Vgf

Scram H2A.Z.1 H2A.Z.2 Scram H2A.Z.1 H2A.Z.2 $\frac{\text { shRNA KD KD }}{\text { TTX (48 hours) }} \frac{\text { shRNA KD } K D}{\text { TTX washout }(60 \mathrm{~min})}$

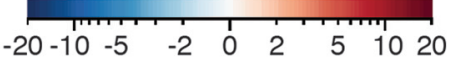

C

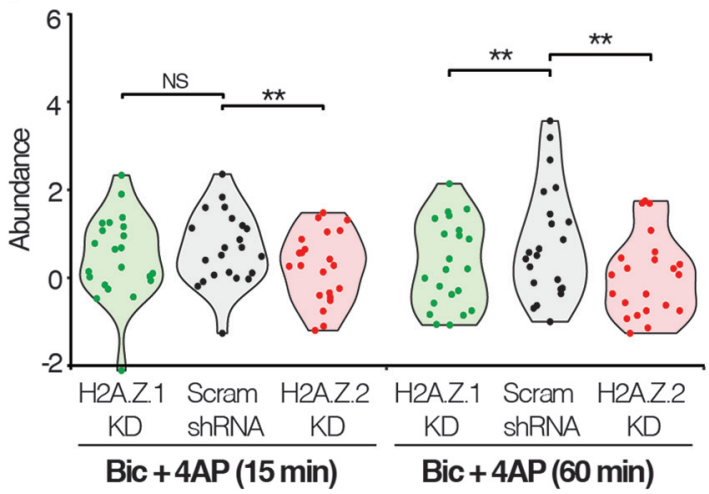

D

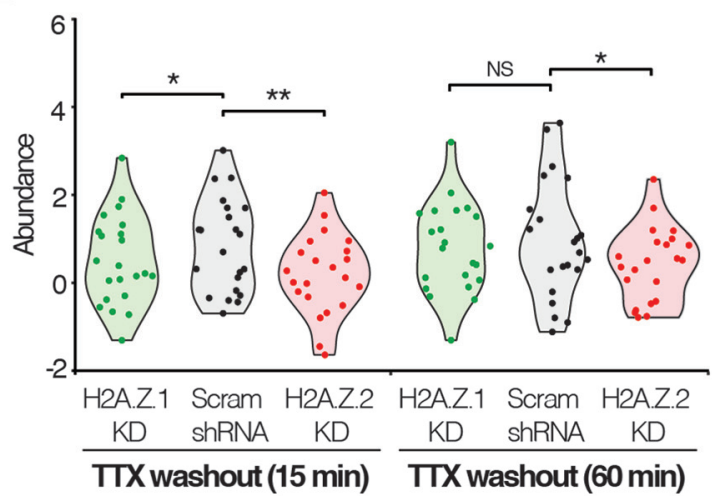

Figure 11. Effect of H2A.Z hypervariant depletion on transcription of delayed IEGs. $\boldsymbol{A}$, Nanostring multiplexed gene expression data (heat maps) representing pre-mRNA levels in control and hypervariant-depleted neurons treated with Bic $+4 \mathrm{AP}$ for indicated times. $\boldsymbol{B}$, Heat map representing pre-mRNA levels in control and hypervariant-depleted neurons treated TTX for $48 \mathrm{~h}$ followed by washout and collected at indicated times. $\boldsymbol{C}, \boldsymbol{D}$, Violin plots depicting pre-mRNA abundance of all delayed IEGs from hypervariant-depleted neurons compared to that of control neurons treated with Bic $+4 A P(C)$ or TTX for $48 \mathrm{~h}$ followed by washout $(\boldsymbol{D})$ for indicated times. $N=3$ for each treatment at all time points. $* p<0.05$ and $* * p<0.01$ (Wilcoxon paired nonparametric test).

but not H2A.Z.1, is required near the Arc TSS for RNA Pol II pausing; (6) activity-induced transcription of certain IEGs is sensitive to depletion of only one of the two hypervariants (e.g., Btg2 and Dusp1 response is less after H2A.Z.2, but not H2A.Z.1, knockdown); and (7) certain IEGs appear to be differentially sensitive to one or both hypervariants in a context-dependent manner (Fbxo33 and Maff after H2A.Z.1 depletion, Fam46a after loss of H2A.Z.2). Taken together, our data strongly suggest that $\mathrm{H} 2 \mathrm{~A} . \mathrm{Z}$ hypervariants have important, nonredundant roles and/or functional specificity that should be considered for a full understanding of the part played by H2A.Z in gene transcription. 
Three lines of evidence inspired our hypothesis about functional specificity of H2A.Z hypervariants. First, several instances have been observed in nature where genes duplicated during evolution encode very similar protein products that have achieved functional diversity. An important example is found in yeast where several paralogous ribosomal proteins are functionally dissimilar to their nearly identical counterparts (Komili et al., 2007; Steffen et al., 2012). Because mechanisms that work are often preserved in nature, we reasoned that the three amino acid differences in these hypervariants might be enough to allow functional diversity. Second, all three variant amino acid residues reside in functionally pertinent regions thereby making them suitable to mediate alternative functionalities. H2A.Z.1 $1^{14 \mathrm{~S}} / \mathrm{H} 2 \mathrm{~A} \cdot Z .2^{14 \mathrm{~A}}$ is located in the $\mathrm{H} 2 \mathrm{~A} . \mathrm{Z} \mathrm{N}$-terminal tail, which is heavily acetylated and is linked to H2A.Z transcriptional activity (Bruce et al., 2005; Valdés-Mora et al., 2012). H2A.Z.1 ${ }^{38 T} / \mathrm{H} 2 \mathrm{~A} . Z .2^{385}$ is located at the edge of the L1 loop, which plays an essential part in intranucleosomal interactions of H2A.Z (Horikoshi et al., 2013). Such intranucleosomal interactions are also facilitated by the unstructured region of H2A.Z C-terminus (Wratting et al., 2012), which houses the H2A.Z.112 ${ }^{128 \mathrm{~V}}$ / H2A.Z.2 ${ }^{128 \mathrm{~A}}$ variation. Third, knockout of H2A.Z.1 results in embryonic lethality in mice (Faast et al., 2001) indicating that H2A.Z.2 cannot compensate for the loss of its paralog. Together, these observations suggest that H2A.Z hypervariants have nonredundant functions that have not yet been adequately explored.

Our study into functional specificity of $\mathrm{H} 2 \mathrm{~A}$.Z hypervariants in activity-induced Arc transcription revealed a few interesting aspects of H2A.Z biology. Overall, Arc transcription requires both H2A.Z.1 and H2A.Z.2. Depletion of either hypervariant had unique effects on Arc transcriptional kinetics that were not compensated by the other paralog. In normal previously untreated neurons (i.e., without homeostatic changes induced by silencing), H2A.Z.2, but not H2A.Z.1, was found to be necessary for the rapid phase of activity-induced Arc transcription (within 15 min of treatment) by facilitating RNA Pol II promoter proximal pausing (Fig. 7). Pol II pausing near promoters is facilitated, among other factors, by the position of the +1 nucleosome (Kwak and Lis, 2013; Jimeno-González et al., 2015). Although exact mechanisms remain unknown, it is presumed that the +1 nucleosome acts as a physical barrier to the transcriptionally engaged Pol II. In mammals, the presence of H2A.Z in the +1 nucleosome is positively correlated with Pol II pausing (Ku et al., 2012; Day et al., 2016). In our study, considering that (1) H2A.Z.2 is required for Pol II pausing near the Arc TSS and (2) Bic + 4AP treatment removes MYC-H2A.Z.2 from the +1 nucleosome (Fig. 6), we suggest that H2A.Z.2-containing nucleosomes play a dual role in mediating activity-induced transcription. In the resting state before activity, the $+1 \mathrm{H} 2 \mathrm{~A}$. Z.2-containing nucleosome would maintain a rigid barrier to Pol II, thereby facilitating its pausing. After activity, dynamic turnover of this nucleosome allows Pol II to commence productive elongation. If true, such a dual-role model could fuel further queries about possible activity-induced signals that convert a rigid nucleosomal barrier into a dynamic gate for Pol II productive elongation. Although this model holds true for activity induced under both normal and prolonged TTX treatment-enabled conditions for Arc, further investigation into other IEGs revealed the potential for both gene- and context-specific roles of H2A.Z hypervariants in gene transcription. However, future studies will be required to determine if these hypervariants are directly or indirectly involved in the transcription of other IEGs investigated in this study.

One of our observations was puzzling and therefore difficult to interpret: although there was turnover of MYCH2A.Z.2 in response to acute increases in neuronal activity, the total levels of H2A.Z (as reported by the Millipore anti-H2A.Z antibody) near the Arc TSS did not change over the entire duration of testing. This could be due to weak efficiency of the commercial H2A.Z antibody (Fig. $6 B$, compare signals), which was the best anti-H2A.Z ChIP antibody in our hands. We believe that, as has been previously reported (Zovkic et al., 2014) and is also shown by our anti-MYC ChIP data, activity does indeed induce H2A.Z turnover at the Arc TSS.

In comparison to the Pol II pausing-related defined role of H2A.Z.2 in Arc transcription, the exact role of H2A.Z.1 in the process is less apparent. In normal, unsilenced neurons, lack of H2A.Z.1 impairs only the late segment of activity-induced $\operatorname{Arc}$ transcription (30 min and later). As suggested by our DRB washout assays, this is most likely via indirect mechanisms (discussed further below). However, after subjecting neurons to homeostatic plasticityrelated changes with TTX exposure for $48 \mathrm{~h}, \mathrm{H} 2 \mathrm{~A} . \mathrm{Z} .1$ depletion also impairs early responses of Arc transcription. This repurposing of H2A.Z.1 after prolonged lack of activity is likely mediated by a context-dependent newfound ability to facilitate Pol II pausing. Alternatively, these data could also be explained by heterogeneity of nucleosomal composition in the cell population (discussed further below).

Considering that we could not detect strong interactions of tagged H2A.Z.1 and H2A.Z.2 in our co-IPs (Fig. 5), indicating that they likely do not often coexist in the same nucleosome, and yet show H2A.Z.1 and H2A.Z.2 enrichment at the same nucleosomal positions (Fig. 6B), it is possible that these Arc TSS nucleosomal loci are populated by different H2A.Z hypervariants in different alleles and/or cells in the population. In normal neurons, only the fraction of these alleles and/or cells that have H2A.Z.2, and therefore paused Pol II, responds to activity. After prolonged TTX treatment, additional alleles and/or cells with H2A.Z.1 attain Pol II pausing via unknown mechanisms and facilitate an enhanced response after TTX washout. This explanation aligns with the noticeably more robust expression of Arc after prolonged TTX treatment and washout in comparison to disinhibition-induced activity under previously untreated conditions (compare Figs. $4 B, 8 B$ ). Also, the effect of H2A.Z.1 depletion on late, not early, Arc transcriptional response (Fig. $4 B, D$ ) falls within the scope of this explanation. On induction of activity in resting conditions, H2A.Z.1-containing alleles and/or cells undertake Arc transcription in a Pol II pausing- 
independent "delayed" fashion, as delayed IEGs do (Saha et al., 2011), thereby accounting for impairments at later time points in H2A.Z.1-depleted neurons. These are exciting possibilities that will require further probing.

Additional experiments will also be required to understand the relevance of the H2A.Z chaperone ANP32E in regulating H2A.Z functions (Mao et al., 2014; Obri et al., 2014). In our hands, several traditional "rescue" attempts involving expression of shRNA-insensitive H2A.Z.2 failed to reverse the loss of Arc transcription in H2A.Z.2-depleted neurons. However, the phenotype loss could be partially rescued by codepletion of ANP32E. Because ANP32E removes H2A.Z from nucleosomes (Mao et al., 2014; Obri et al., 2014; Gursoy-Yuzugullu et al., 2015), it is likely that the presence of ANP32E in H2A.Z.2-depleted neurons facilitates continued removal of nucleosomal H2A.Z.2 and subsequent fallouts thereof, including loss of Pol II pausing and inability to respond rapidly. However, codepletion of the chaperone along with the histone preserves nucleosomal H2A.Z.2, at least in part, enabling Arc to rapidly respond to some extent. A similar precedence of H2A.Z and ANP32E codepletion partially rescuing phenotype loss due to depletion of one of these factors exists in non-neuronal cells (Alatwi and Downs, 2015). Our findings, combined with this previous report, advocate for the need to approach the complex biology of H2A.Z hypervariants and their chaperones together.
Collectively, our current studies indicate that H2A.Z hypervariants mediate activity-induced IEG transcription in a context-dependent (H2A.Z.1) or context-independent (H2A.Z.2) fashion, where the extent of their involvement in the transcriptional process varies from gene to gene. Our data also suggest spatial and temporal cooperation of these hypervariants in manifesting the maximum gene response under a given circumstance. Natural extensions of the current work will be to test the above hypothesis about allele/cell-specificity of these hypervariants, and to investigate molecular mechanisms connecting H2A.Z hypervariants and paused Pol II. Additionally, sequentially mutating H2A.Z.1 to H2A.Z.2 could provide insight into which amino acid differences are necessary for functional specificity. Particular attention may be paid to the H2A.Z. $1^{14 \mathrm{~S}} / \mathrm{H} 2 \mathrm{~A} . Z .2^{14 \mathrm{~A}}$ variation, where H2A.Z.1, but not H2A.Z.2, could be potentially phosphorylated. It will also be of interest to learn about hypervariant-specific H2A.Z chaperones, if any, and understand how these hypervariants differentially regulate expression of several crucial synaptic molecules. Finally, it is our hope that the data presented here will increase appreciation for functional specificity of H2A.Z hypervariants and promote toolbuilding efforts to enable a comprehensive understanding of diverse roles played by these paralogs at different genes and under different neurobiological contexts.

Table 2. List of DEGs after H2A.Z.1 and H2A.Z.2 KD-part A (upregulated after H2A.Z.1 KD)

\begin{tabular}{|c|c|c|c|c|c|}
\hline Symbol & Scrambled expression & shH2AZ.1 expression & log2 fold change & $p$ value & FDR \\
\hline Ace & 4.140032802 & 5.231924578 & 1.091891777 & $2.67 \mathrm{E}-06$ & 0.049404732 \\
\hline BF565662 & 3.628967203 & 5.376799174 & 1.747831971 & $1.69 \mathrm{E}-05$ & 0.065026891 \\
\hline TC524990 & 8.870590012 & 10.29163907 & 1.421049062 & $1.88 \mathrm{E}-05$ & 0.065026891 \\
\hline Ccdc23 & 14.47197211 & 15.09673068 & 0.624758569 & $2.34 \mathrm{E}-05$ & 0.065026891 \\
\hline RGD1307943_predicted & 5.328316737 & 7.637953753 & 2.309637017 & $3.41 \mathrm{E}-05$ & 0.076745729 \\
\hline RGD1563203_predicted & 10.11295573 & 10.66114527 & 0.548189542 & $3.52 \mathrm{E}-05$ & 0.076745729 \\
\hline AW918709 & 11.15514358 & 12.49064688 & 1.335503299 & $4.75 \mathrm{E}-05$ & 0.07711271 \\
\hline Ddx39 & 11.53226567 & 12.3812449 & 0.848979229 & $5.93 E-05$ & 0.07711271 \\
\hline CX570116 & 8.384790847 & 8.916443761 & 0.531652913 & $6.90 \mathrm{E}-05$ & 0.07711271 \\
\hline $\mathrm{BI} 293610$ & 9.493734401 & 9.936922735 & 0.443188333 & $7.00 \mathrm{E}-05$ & 0.07711271 \\
\hline ENSRNOT00000013636 & 10.29622089 & 11.25318643 & 0.956965537 & $7.45 \mathrm{E}-05$ & 0.07711271 \\
\hline Slc13a5 & 8.198920861 & 9.995278642 & 1.796357781 & $7.61 \mathrm{E}-05$ & 0.07711271 \\
\hline ENSRNOT00000028053 & 6.936499815 & 7.576197512 & 0.639697697 & 7.67E-05 & 0.07711271 \\
\hline Ddc & 6.457510865 & 8.216773518 & 1.759262653 & $7.75 \mathrm{E}-05$ & 0.07711271 \\
\hline Hdmcp & 6.071940673 & 7.657559709 & 1.585619036 & $8.10 \mathrm{E}-05$ & 0.07711271 \\
\hline СА339586 & 11.74191409 & 12.75911189 & 1.01719779 & $9.56 \mathrm{E}-05$ & 0.07711271 \\
\hline Prlr & 3.864580889 & 4.720040256 & 0.855459367 & $9.71 \mathrm{E}-05$ & 0.07711271 \\
\hline TC533299 & 10.13831539 & 10.92955405 & 0.791238655 & $9.94 \mathrm{E}-05$ & 0.07711271 \\
\hline DV722215 & 4.7082684 & 6.286053239 & 1.577784839 & 0.000100574 & 0.07711271 \\
\hline TC538999 & 5.251634416 & 6.808826043 & 1.557191627 & 0.000112895 & 0.07711271 \\
\hline Ube2i & 11.90457203 & 12.34706984 & 0.442497806 & 0.00013949 & 0.07711271 \\
\hline BF542749 & 10.74023303 & 11.68091656 & 0.940683533 & 0.000154434 & 0.07711271 \\
\hline Ext2_predicted & 10.8894966 & 11.22399898 & 0.334502379 & 0.000161949 & 0.07711271 \\
\hline RGD1311752_predicted & 8.703882692 & 10.44148626 & 1.737603564 & 0.000169954 & 0.07711271 \\
\hline TC522315 & 2.79964542 & 4.101760357 & 1.302114937 & 0.000179121 & 0.07711271 \\
\hline AA942848 & 8.500936128 & 9.102749214 & 0.601813086 & 0.000182133 & 0.07711271 \\
\hline RGD1308031 & 5.736834927 & 6.676339719 & 0.939504792 & 0.000186685 & 0.07711271 \\
\hline Raph1_predicted & 6.652550515 & 9.501294881 & 2.848744366 & 0.000196584 & 0.07711271 \\
\hline Col5a3 & 9.457522664 & 10.38121689 & 0.923694229 & 0.000206023 & 0.07711271 \\
\hline BF523017 & 10.85115842 & 11.3588647 & 0.507706279 & 0.000206202 & 0.07711271 \\
\hline CB547155 & 9.262431376 & $\begin{array}{l}9.602202763 \\
\text { (Continued) }\end{array}$ & 0.339771388 & 0.000218209 & 0.07711271 \\
\hline
\end{tabular}


Table 2. Continued

\begin{tabular}{|c|c|c|c|c|c|}
\hline Symbol & Scrambled expression & shH2AZ.1 expression & log2 fold change & $p$ value & FDR \\
\hline Pcsk3 & 9.563934918 & 10.34288495 & 0.778950036 & 0.000220488 & 0.07711271 \\
\hline TC545808 & 8.730436041 & 9.609236409 & 0.878800369 & 0.000220793 & 0.07711271 \\
\hline Mfap5_predicted & 5.818035999 & 7.453695841 & 1.635659842 & 0.000225576 & 0.07711271 \\
\hline LOC684302 & 9.817000952 & 10.28928105 & 0.472280097 & 0.000228335 & 0.07711271 \\
\hline BE111891 & 1.08420639 & 1.983656252 & 0.899449863 & 0.000230407 & 0.07711271 \\
\hline Adam3 & 1.795552474 & 4.569825212 & 2.774272738 & 0.00024164 & 0.07711271 \\
\hline RGD1309534 & 8.024579741 & 8.473932865 & 0.449353124 & 0.00025109 & 0.07711271 \\
\hline XM_224155 & 1.757920019 & 4.374588989 & 2.61666897 & 0.000254712 & 0.07711271 \\
\hline Anks1_predicted & 4.785255527 & 5.643215295 & 0.857959768 & 0.00025571 & 0.07711271 \\
\hline TC564875 & 9.056765795 & 9.929394592 & 0.872628797 & 0.000260742 & 0.07711271 \\
\hline Zfand2b & 10.84791412 & 11.55774178 & 0.709827653 & 0.000270732 & 0.07711271 \\
\hline CA333998 & 11.83201426 & 12.28062694 & 0.448612678 & 0.000284527 & 0.07711271 \\
\hline Mfng & 7.749259513 & 8.266369008 & 0.517109494 & 0.000296828 & 0.07711271 \\
\hline Bambi & 8.812389606 & 9.914938916 & 1.10254931 & 0.000302915 & 0.07711271 \\
\hline XM_346066 & 1.076776976 & 2.82468633 & 1.747909353 & 0.000311005 & 0.07711271 \\
\hline Spg21 & 11.23012332 & 11.55721656 & 0.327093237 & 0.000318552 & 0.07711271 \\
\hline CO387496 & 4.453414129 & 6.536747702 & 2.083333573 & 0.000333125 & 0.07711271 \\
\hline TC554267 & 8.373468927 & 9.14689548 & 0.773426553 & 0.000346481 & 0.07711271 \\
\hline Den & 5.529606864 & 8.465423531 & 2.935816667 & 0.000347064 & 0.07711271 \\
\hline RGD1309062 & 6.563975115 & 8.064163786 & 1.500188671 & 0.000352004 & 0.07711271 \\
\hline Gprk6 & 12.47356751 & 13.45420856 & 0.980641054 & 0.000358039 & 0.07711271 \\
\hline RGD1305243_predicted & 7.220436673 & 7.549994374 & 0.329557701 & 0.000358398 & 0.07711271 \\
\hline DV716578 & 8.728614931 & 9.434993476 & 0.706378545 & 0.00036259 & 0.07711271 \\
\hline Lipl3_predicted & 4.392258287 & 6.178702783 & 1.786444496 & 0.000363005 & 0.07711271 \\
\hline Mmp24 & 15.9433976 & 16.61068899 & 0.667291392 & 0.000369777 & 0.07711271 \\
\hline Tmem77 & 9.818583769 & 10.37533496 & 0.55675119 & 0.000374996 & 0.07711271 \\
\hline Ard1_predicted & 11.53917153 & 12.08142809 & 0.542256562 & 0.000383345 & 0.07711271 \\
\hline RGD1566292_predicted & 11.21332683 & 11.62680095 & 0.413474114 & 0.000388946 & 0.07711271 \\
\hline RGD1311331_predicted & 7.875456813 & 8.872995293 & 0.997538481 & 0.000400441 & 0.07711271 \\
\hline BF556192 & 12.80155585 & 13.55394357 & 0.752387717 & 0.000416696 & 0.07711271 \\
\hline Ulk1_mapped & 8.808173483 & 9.951782485 & 1.143609002 & 0.000418969 & 0.07711271 \\
\hline XM_341055 & 6.720993554 & 7.689316998 & 0.968323445 & 0.000434274 & 0.07711271 \\
\hline Pxn & 7.025381997 & 7.696209005 & 0.670827008 & 0.000443884 & 0.07711271 \\
\hline Prdm7_predicted & 8.455624612 & 9.496578258 & 1.040953646 & 0.000449472 & 0.07711271 \\
\hline Sdcbp2 & 3.853370711 & 4.661073334 & 0.807702623 & 0.000449476 & 0.07711271 \\
\hline Nkiras2_predicted & 10.80772401 & 12.34366608 & 1.535942062 & 0.000453754 & 0.07711271 \\
\hline RGD1306939 & 5.039293136 & 5.763551483 & 0.724258347 & 0.000460339 & 0.07711271 \\
\hline BF549650 & 10.67675061 & 11.62149087 & 0.944740258 & 0.000464965 & 0.07711271 \\
\hline Tcte3_predicted & 1.596662365 & 4.522208185 & 2.92554582 & 0.000467652 & 0.07711271 \\
\hline Rhebl1 & 12.07802518 & 12.52188936 & 0.443864183 & 0.000468091 & 0.07711271 \\
\hline RGD1565763_predicted & 3.722812164 & 5.448624313 & 1.72581215 & 0.000488696 & 0.07711271 \\
\hline Setmar & 7.220618123 & 8.453991365 & 1.233373242 & 0.000490118 & 0.07711271 \\
\hline Vwa1 & 6.169798444 & 6.711947958 & 0.542149513 & 0.000494053 & 0.07711271 \\
\hline Limk1 & 13.40359822 & 14.23252143 & 0.82892321 & 0.000495236 & 0.07711271 \\
\hline Adam24_predicted & 1.398107927 & 3.625631203 & 2.227523276 & 0.00049853 & 0.07711271 \\
\hline DV720603 & 12.79953832 & 13.65543137 & 0.855893047 & 0.000504802 & 0.07711271 \\
\hline XM_215391 & 5.439407969 & 6.039017881 & 0.599609912 & 0.000510659 & 0.07711271 \\
\hline LOC̄498368 & 8.728806947 & 9.512459784 & 0.783652838 & 0.0005142 & 0.07711271 \\
\hline Zcwpw1_predicted & 8.549635796 & 9.0135438 & 0.463908005 & 0.000519295 & 0.07711271 \\
\hline RGD1306839_predicted & 13.69986612 & 14.17485686 & 0.474990743 & 0.000521826 & 0.07711271 \\
\hline Itga1 & 8.054333478 & 8.953630387 & 0.899296909 & 0.000524578 & 0.07711271 \\
\hline Al136665 & 2.376789645 & 3.389671123 & 1.012881477 & 0.000524619 & 0.07711271 \\
\hline Pcdh19_predicted & 13.08120857 & 13.962638 & 0.881429428 & 0.000533667 & 0.07711271 \\
\hline BF564180 & 5.808806457 & 6.675428768 & 0.866622311 & 0.000536071 & 0.07711271 \\
\hline TC543571 & 1.274446722 & 2.996226578 & 1.721779856 & 0.000539336 & 0.07711271 \\
\hline Atp1b3 & 12.99375325 & 13.39955207 & 0.405798822 & 0.000540772 & 0.07711271 \\
\hline Kctd10 & 13.95844395 & 14.54894225 & 0.590498302 & 0.000541089 & 0.07711271 \\
\hline RGD1565709_predicted & 2.770943739 & 4.053574585 & 1.282630846 & 0.000544688 & 0.07711271 \\
\hline MGC108896 & 9.927683288 & 10.78529474 & 0.857611447 & 0.00054487 & 0.07711271 \\
\hline Kctd12_predicted & 5.830704506 & 6.729043191 & 0.898338685 & 0.0005464 & 0.07711271 \\
\hline TC544355 & 5.942384994 & 6.836536724 & 0.894151731 & 0.000547615 & 0.07711271 \\
\hline Reps1_predicted & 12.54940511 & 12.97880545 & 0.429400346 & 0.000564254 & 0.077922785 \\
\hline Serpini2 & 3.851552344 & $\begin{array}{r}5.63331535 \\
\text { (Continued) }\end{array}$ & 1.781763007 & 0.000567829 & 0.077922785 \\
\hline
\end{tabular}


Table 2. Continued

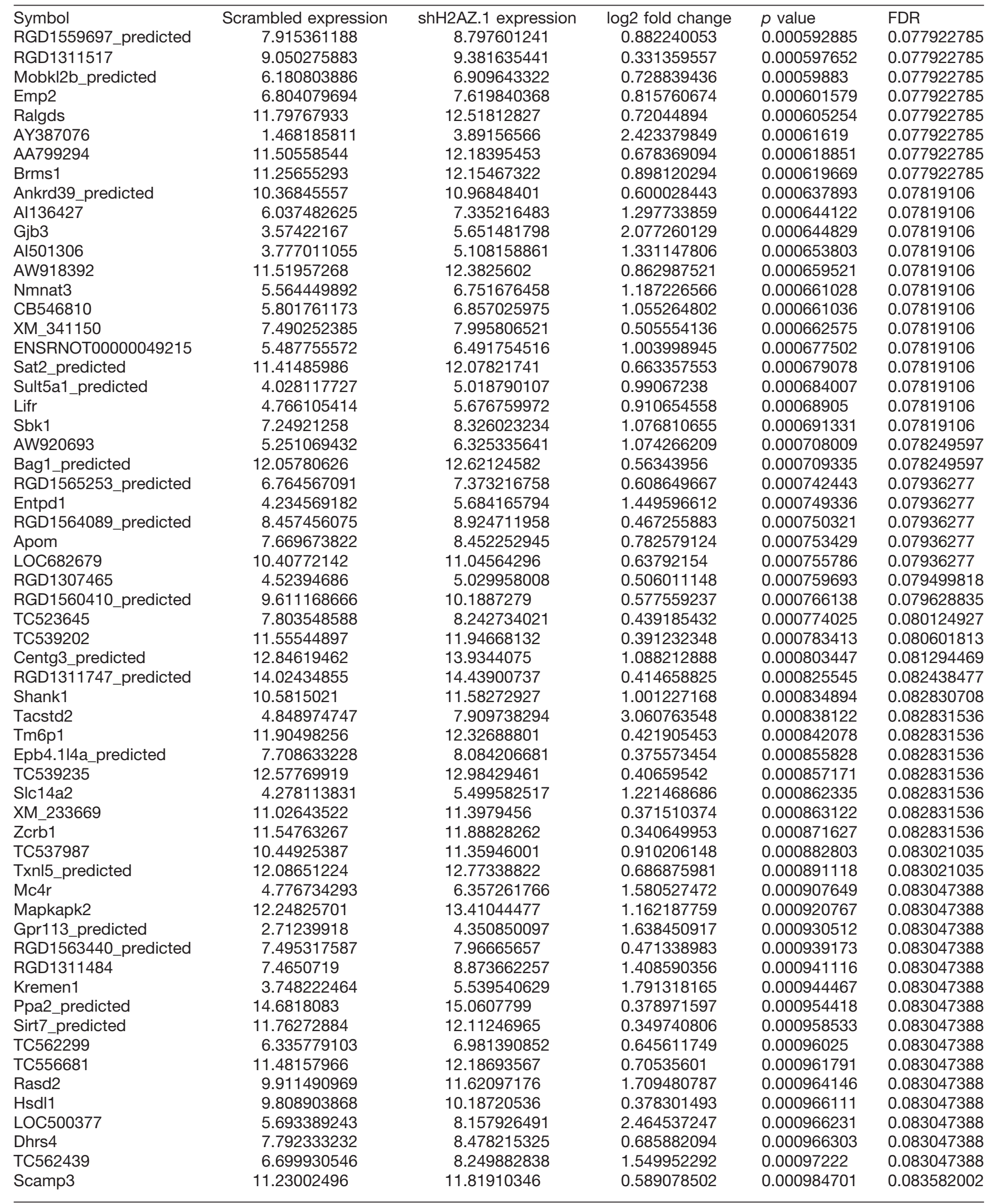


Table 3. List of DEGs after H2A.Z.1 and H2A.Z.2 KD-part B (downregulated after H2A.Z.1 KD)

\begin{tabular}{|c|c|c|c|c|c|}
\hline symbol & scrambled expression & shh2az.1 expression & log2 fold change & $p$ value & fdr \\
\hline loc287522 & 6.259898423 & 3.781938662 & -2.477959761 & $6.60 \mathrm{e}-06$ & 0.049404732 \\
\hline rgd1566064_predicted & 12.81190937 & 9.362864495 & -3.449044872 & $7.18 \mathrm{e}-06$ & 0.049404732 \\
\hline tomm34_predicted & 9.80058324 & 8.124036057 & -1.676547183 & $8.08 e-06$ & 0.049404732 \\
\hline ck363699 & 6.61546991 & 5.78793267 & -0.827537241 & $1.87 \mathrm{e}-05$ & 0.065026891 \\
\hline bf562307 & 9.734906301 & 8.443322223 & -1.291584078 & $2.29 \mathrm{e}-05$ & 0.065026891 \\
\hline tc553984 & 10.57601225 & 9.76833986 & -0.807672386 & $2.34 \mathrm{E}-05$ & 0.065026891 \\
\hline Al599250 & 7.713399206 & 7.087788209 & -0.625610997 & $3.23 \mathrm{E}-05$ & 0.076745729 \\
\hline BC098774 & 7.237548308 & 5.570550667 & -1.666997641 & 4.77E-05 & 0.07711271 \\
\hline Grik4 & 9.223661505 & 8.523675197 & -0.699986308 & $5.03 \mathrm{E}-05$ & 0.07711271 \\
\hline Sdhd & 14.37797406 & 13.56563435 & -0.812339706 & $6.16 \mathrm{E}-05$ & 0.07711271 \\
\hline Napg & 10.37368659 & 9.853267401 & -0.520419193 & $6.42 \mathrm{E}-05$ & 0.07711271 \\
\hline Mrrf & 7.127263566 & 6.065031172 & -1.062232394 & 8.17E-05 & 0.07711271 \\
\hline BC091405 & 10.38125201 & 9.282285641 & -1.09896637 & $8.74 \mathrm{E}-05$ & 0.07711271 \\
\hline Tarbp2 & 12.45655726 & 12.07159614 & -0.384961117 & 9.39E-05 & 0.07711271 \\
\hline RGD1309546_predicted & 8.534002377 & 7.502899404 & -1.031102973 & 0.000106301 & 0.07711271 \\
\hline Grin2c & 8.899255961 & 6.97904754 & -1.920208421 & 0.000106996 & 0.07711271 \\
\hline TC566987 & 6.659279818 & 5.827413166 & -0.831866652 & 0.000108607 & 0.07711271 \\
\hline TC552897 & 5.933248175 & 4.801232308 & -1.132015867 & 0.00010879 & 0.07711271 \\
\hline Prkar2a & 6.163801451 & 5.313528282 & -0.850273169 & 0.000111853 & 0.07711271 \\
\hline Glt1d1_predicted & 5.121800999 & 4.103526035 & -1.018274964 & 0.000112311 & 0.07711271 \\
\hline BF555161 & 10.59364955 & 9.470574079 & -1.123075469 & 0.000117617 & 0.07711271 \\
\hline TC538830 & 12.53146816 & 11.24170839 & -1.289759763 & 0.000121745 & 0.07711271 \\
\hline RGD1566054_predicted & 9.115400025 & 8.101685773 & -1.013714251 & 0.000122074 & 0.07711271 \\
\hline Lrp11_predicted & 13.07487226 & 11.70424527 & -1.370626989 & 0.000124681 & 0.07711271 \\
\hline TC536024 & 7.13278452 & 6.240473074 & -0.892311445 & 0.000125993 & 0.07711271 \\
\hline Cited2 & 10.85269214 & 8.990111494 & -1.862580645 & 0.000136342 & 0.07711271 \\
\hline BF397813 & 7.19066408 & 5.792890555 & -1.397773525 & 0.000144061 & 0.07711271 \\
\hline Al112975 & 6.352713186 & 5.377218263 & -0.975494923 & 0.000146482 & 0.07711271 \\
\hline RGD1566367_predicted & 5.6358019 & 5.240674268 & -0.395127632 & 0.00014795 & 0.07711271 \\
\hline Polr3h_predicted & 11.44293632 & 10.23386016 & -1.209076162 & 0.000150598 & 0.07711271 \\
\hline Pygb & 12.45213506 & 11.79157662 & -0.660558447 & 0.000154638 & 0.07711271 \\
\hline AW142634 & 8.922013872 & 7.829003261 & -1.093010611 & 0.000156724 & 0.07711271 \\
\hline BF565628 & 10.29869369 & 9.349174788 & -0.949518899 & 0.000157086 & 0.07711271 \\
\hline Gdap1_predicted & 8.365334903 & 6.792027238 & -1.573307664 & 0.000164033 & 0.07711271 \\
\hline Ryr2 & 8.079272174 & 7.27621553 & -0.803056644 & 0.000164158 & 0.07711271 \\
\hline BF555945 & 7.644955812 & 5.93488388 & -1.710071931 & 0.000166291 & 0.07711271 \\
\hline Chchd1_predicted & 13.92529609 & 13.52993193 & -0.395364161 & 0.000185585 & 0.07711271 \\
\hline CB718612 & 8.141380063 & 7.317726555 & -0.823653508 & 0.00018998 & 0.07711271 \\
\hline BF401583 & 3.807342642 & 3.135350749 & -0.671991893 & 0.000196042 & 0.07711271 \\
\hline DV718172 & 13.52733676 & 12.61872235 & -0.908614414 & 0.000205766 & 0.07711271 \\
\hline AW921183 & 10.34179328 & 9.467173785 & -0.874619492 & 0.000208601 & 0.07711271 \\
\hline Atp6v1a1_predicted & 10.42042831 & 10.06973958 & -0.350688726 & 0.000209702 & 0.07711271 \\
\hline Hexim2_predicted & 9.914767395 & 9.287364476 & -0.627402919 & 0.000237955 & 0.07711271 \\
\hline Pigh_predicted & 6.450865425 & 5.643206561 & -0.807658864 & 0.000246319 & 0.07711271 \\
\hline LOC307783 & 5.032882194 & 3.665875684 & -1.367006509 & 0.000248578 & 0.07711271 \\
\hline LOC690163 & 8.287898848 & 6.848782046 & -1.439116802 & 0.000252011 & 0.07711271 \\
\hline RGD1566086_predicted & 6.787042716 & 6.316220869 & -0.470821846 & 0.000252689 & 0.07711271 \\
\hline TC525615 & 11.81161754 & 10.5813283 & -1.230289236 & 0.000256806 & 0.07711271 \\
\hline Pou3f1 & 10.95885519 & 9.374839483 & -1.584015704 & 0.000261757 & 0.07711271 \\
\hline AW920545 & 8.612227937 & 8.006194471 & -0.606033466 & 0.000263301 & 0.07711271 \\
\hline TC530561 & 6.361469677 & 4.977425946 & -1.38404373 & 0.000266017 & 0.07711271 \\
\hline Vps45 & 11.18560958 & 10.64488764 & -0.540721933 & 0.000266506 & 0.07711271 \\
\hline RGD1565289_predicted & 13.51664684 & 12.46921634 & -1.047430504 & 0.000283049 & 0.07711271 \\
\hline KIhl20_predicted & 8.808515699 & 7.624582956 & -1.183932742 & 0.000283307 & 0.07711271 \\
\hline Pdzk8_predicted & 8.242171572 & 7.347607077 & -0.894564495 & 0.000305242 & 0.07711271 \\
\hline TC560958 & 11.93185862 & 11.11468764 & -0.817170979 & 0.000305894 & 0.07711271 \\
\hline Cops7a_predicted & 13.1106929 & 12.4375094 & -0.673183498 & 0.000306494 & 0.07711271 \\
\hline TC528997 & 9.156193399 & 8.115543858 & -1.040649541 & 0.000308314 & 0.07711271 \\
\hline RGD1310351_predicted & 10.85564614 & 9.902288125 & -0.953358011 & 0.00031047 & 0.07711271 \\
\hline Pdcd8 & 12.75065713 & 12.03492925 & -0.715727881 & 0.00031379 & 0.07711271 \\
\hline Ica1 & 12.17364623 & 11.6622667 & -0.511379533 & 0.000314358 & 0.07711271 \\
\hline LOC680426 & 11.2010015 & $\begin{array}{c}10.36982724 \\
\text { (Continued) }\end{array}$ & -0.831174259 & 0.000315713 & 0.07711271 \\
\hline
\end{tabular}


Table 3. Continued

\begin{tabular}{|c|c|c|c|c|c|}
\hline Symbol & Scrambled expression & shH2AZ.1 expression & log2 fold change & $p$ value & FDR \\
\hline DV719080 & 10.87559452 & 9.495677787 & -1.379916733 & 0.000318635 & 0.07711271 \\
\hline TC545343 & 7.438383629 & 6.879531146 & -0.558852483 & 0.000332478 & 0.07711271 \\
\hline Gsta3 & 12.17020876 & 10.20865792 & -1.961550836 & 0.00033429 & 0.07711271 \\
\hline Mfn2 & 10.55727808 & 10.01586245 & -0.541415627 & 0.000342094 & 0.07711271 \\
\hline AABR03068037 & 7.785306555 & 7.319303528 & -0.466003028 & 0.000351693 & 0.07711271 \\
\hline BE113961 & 10.80779587 & 9.878175532 & -0.92962034 & 0.00035311 & 0.07711271 \\
\hline TC519727 & 11.59791019 & 10.81753792 & -0.780372272 & 0.000357927 & 0.07711271 \\
\hline TC528585 & 7.453045217 & 6.948322568 & -0.504722649 & 0.000359377 & 0.07711271 \\
\hline RGD1311345 & 8.400406852 & 7.683659105 & -0.716747748 & 0.000361038 & 0.07711271 \\
\hline TC543044 & 11.15897338 & 10.80731226 & -0.351661122 & 0.00036687 & 0.07711271 \\
\hline TC538360 & 10.10529859 & 9.492938508 & -0.612360085 & 0.000369532 & 0.07711271 \\
\hline RGD1306053 & 14.18807422 & 12.78633165 & -1.401742574 & 0.00037825 & 0.07711271 \\
\hline Tmem55a & 10.29421099 & 9.628109886 & -0.666101101 & 0.000385931 & 0.07711271 \\
\hline CK478004 & 4.998539607 & 4.108978333 & -0.889561275 & 0.000389178 & 0.07711271 \\
\hline Cog6 & 8.794988406 & 7.924249265 & -0.870739141 & 0.000390394 & 0.07711271 \\
\hline TC544658 & 11.70378121 & 11.34008183 & -0.363699382 & 0.000392004 & 0.07711271 \\
\hline AA957814 & 7.99801766 & 6.667451213 & -1.330566447 & 0.000392693 & 0.07711271 \\
\hline AA851065 & 10.69658123 & 9.076743189 & -1.619838043 & 0.000397605 & 0.07711271 \\
\hline CB545107 & 10.80488687 & 9.566124168 & -1.2387627 & 0.000400668 & 0.07711271 \\
\hline BP503923 & 11.97203025 & 10.95266512 & -1.019365134 & 0.000411009 & 0.07711271 \\
\hline Dnajc8 & 11.88375963 & 11.47580858 & -0.407951048 & 0.000412706 & 0.07711271 \\
\hline Grin2b & 7.819443318 & 6.894573198 & -0.92487012 & 0.000417686 & 0.07711271 \\
\hline Slc16a11_predicted & 10.74873215 & 9.354260934 & -1.394471217 & 0.000419341 & 0.07711271 \\
\hline Birc6_predicted & 7.745470898 & 7.220010389 & -0.525460509 & 0.000419662 & 0.07711271 \\
\hline Mib1_predicted & 8.245212972 & 7.371903929 & -0.873309043 & 0.000423797 & 0.07711271 \\
\hline TC559433 & 11.13137332 & 9.782275241 & -1.349098076 & 0.000423917 & 0.07711271 \\
\hline AW916157 & 11.73649748 & 10.69087852 & -1.04561896 & 0.000426872 & 0.07711271 \\
\hline AW917472 & 11.34281752 & 10.58589712 & -0.756920404 & 0.000435355 & 0.07711271 \\
\hline Tap1 & 9.061885637 & 8.182214145 & -0.879671492 & 0.000440342 & 0.07711271 \\
\hline AW916613 & 2.654368574 & 1.390282852 & -1.264085722 & 0.000444876 & 0.07711271 \\
\hline Mfsd1_predicted & 6.848503926 & 5.756632868 & -1.091871059 & 0.000445474 & 0.07711271 \\
\hline AA818474 & 4.391921753 & 3.594459458 & -0.797462295 & 0.000446082 & 0.07711271 \\
\hline TC563567 & 8.989583387 & 8.005430887 & -0.9841525 & 0.000455838 & 0.07711271 \\
\hline RGD1306356 & 10.99949907 & 10.38002044 & -0.61947863 & 0.000463089 & 0.07711271 \\
\hline DV715341 & 8.050123212 & 7.619079779 & -0.431043434 & 0.000473159 & 0.07711271 \\
\hline BF563211 & 6.051837901 & 5.373536893 & -0.678301008 & 0.000476289 & 0.07711271 \\
\hline Rims1 & 7.088218226 & 6.595183902 & -0.493034323 & 0.000480843 & 0.07711271 \\
\hline Al102821 & 12.8265747 & 11.10486978 & -1.721704924 & 0.000496254 & 0.07711271 \\
\hline Umps & 10.19219914 & 9.598751948 & -0.593447192 & 0.000499689 & 0.07711271 \\
\hline Al175475 & 6.709628141 & 5.644117818 & -1.065510323 & 0.00050039 & 0.07711271 \\
\hline Nek6 & 8.871652763 & 7.678760217 & -1.192892546 & 0.000500809 & 0.07711271 \\
\hline LOC290577 & 8.796783148 & 8.098975545 & -0.697807603 & 0.000512747 & 0.07711271 \\
\hline Epb4.1l1 & 12.87470645 & 11.8197883 & -1.054918153 & 0.000513513 & 0.07711271 \\
\hline AW917204 & 10.32038964 & 8.851718495 & -1.468671143 & 0.000518431 & 0.07711271 \\
\hline Aggf1 & 8.624603215 & 7.155915536 & -1.468687679 & 0.00052289 & 0.07711271 \\
\hline TC524569 & 8.967339631 & 8.112362678 & -0.854976953 & 0.000530873 & 0.07711271 \\
\hline Acsbg1 & 14.91888151 & 14.34065365 & -0.578227868 & 0.000532623 & 0.07711271 \\
\hline TC532756 & 7.481185857 & 6.76026886 & -0.720916997 & 0.000537663 & 0.07711271 \\
\hline AW918535 & 14.8762029 & 14.0293993 & -0.846803594 & 0.000543389 & 0.07711271 \\
\hline TC547881 & 8.611169112 & 7.435283572 & -1.17588554 & 0.000544447 & 0.07711271 \\
\hline TC525657 & 7.989864402 & 6.913160036 & -1.076704366 & 0.000558529 & 0.077922785 \\
\hline Dlst & 14.58436665 & 14.05417512 & -0.530191529 & 0.000568429 & 0.077922785 \\
\hline BE117446 & 4.271387612 & 3.642009885 & -0.629377726 & 0.000571062 & 0.077922785 \\
\hline TC556147 & 11.20330191 & 9.935224997 & -1.268076913 & 0.000571171 & 0.077922785 \\
\hline Rbm18_predicted & 8.265474859 & 7.532437984 & -0.733036875 & 0.000573185 & 0.077922785 \\
\hline Chgb & 16.35659504 & 15.63431894 & -0.722276099 & 0.000576277 & 0.077922785 \\
\hline LOC682205 & 8.396143751 & 7.517812838 & -0.878330913 & 0.000576944 & 0.077922785 \\
\hline Gpr173 & 6.652605716 & 5.860519811 & -0.792085905 & 0.000590354 & 0.077922785 \\
\hline XM_216096 & 7.832298665 & 6.566407732 & -1.265890933 & 0.000599328 & 0.077922785 \\
\hline Рpp6c & 9.764572047 & 9.414556134 & -0.350015913 & 0.000605339 & 0.077922785 \\
\hline XM_343871 & 10.90379578 & 10.24265728 & -0.661138501 & 0.000606072 & 0.077922785 \\
\hline Fgd4 & 8.357984576 & $\begin{array}{l}7.228528075 \\
\quad \text { (Continued) }\end{array}$ & -1.129456501 & 0.000610696 & 0.077922785 \\
\hline
\end{tabular}


Table 3. Continued

\begin{tabular}{|c|c|c|c|c|c|}
\hline Symbol & Scrambled expression & shH2AZ.1 expression & log2 fold change & $p$ value & FDR \\
\hline CX569250 & 9.795475601 & 9.227937407 & -0.567538194 & 0.00061442 & 0.077922785 \\
\hline Hap1 & 12.02268819 & 11.01938568 & -1.003302507 & 0.000616681 & 0.077922785 \\
\hline AW915320 & 9.170075196 & 8.417656657 & -0.752418539 & 0.000618451 & 0.077922785 \\
\hline Rragd_predicted & 10.70444865 & 9.093806459 & -1.610642189 & 0.000619331 & 0.077922785 \\
\hline TC541972 & 10.34640635 & 9.316275627 & -1.030130724 & 0.00063095 & 0.07819106 \\
\hline $\mathrm{Al} 230360$ & 7.918189901 & 7.234716989 & -0.683472912 & 0.000642562 & 0.07819106 \\
\hline $\operatorname{lgf} 2 r$ & 13.17561599 & 12.47214529 & -0.703470695 & 0.000648788 & 0.07819106 \\
\hline DV719617 & 10.27750625 & 9.107724317 & -1.169781934 & 0.000651766 & 0.07819106 \\
\hline Mrpl18_predicted & 12.51441646 & 11.58262484 & -0.931791612 & 0.000662315 & 0.07819106 \\
\hline TC568517 & 7.18069051 & 6.439772542 & -0.740917968 & 0.000674076 & 0.07819106 \\
\hline Adsl_predicted & 12.53997034 & 11.84135281 & -0.698617527 & 0.000679503 & 0.07819106 \\
\hline TC519890 & 15.20193502 & 13.84576879 & -1.356166231 & 0.000682539 & 0.07819106 \\
\hline Spg20 & 8.308415518 & 7.58305852 & -0.725356998 & 0.000686525 & 0.07819106 \\
\hline Al170696 & 6.942817153 & 6.027001338 & -0.915815815 & 0.00069311 & 0.07819106 \\
\hline Ube2v2 & 9.661199706 & 8.572804044 & -1.088395662 & 0.000693451 & 0.07819106 \\
\hline LOC498295 & 12.20451881 & 11.83959221 & -0.364926599 & 0.000698494 & 0.078249597 \\
\hline TC560921 & 5.207732953 & 4.297097796 & -0.910635157 & 0.000700069 & 0.078249597 \\
\hline BF555924 & 9.985597821 & 9.469135844 & -0.516461977 & 0.000702983 & 0.078249597 \\
\hline Pfkm & 14.51654843 & 14.05002374 & -0.466524695 & 0.000708505 & 0.078249597 \\
\hline TC564512 & 8.333396639 & 7.32224411 & -1.011152528 & 0.000721932 & 0.07899534 \\
\hline LOC500295 & 9.420651641 & 8.540467639 & -0.880184003 & 0.000724825 & 0.07899534 \\
\hline Sf3b2_predicted & 12.44354911 & 11.6085621 & -0.83498701 & 0.000725793 & 0.07899534 \\
\hline BF523428 & 13.15045896 & 12.09237333 & -1.058085628 & 0.000726436 & 0.07899534 \\
\hline Zfp297b & 8.14767076 & 7.796674244 & -0.350996515 & 0.000734886 & 0.07936277 \\
\hline AA998677 & 6.022499947 & 5.66326418 & -0.359235767 & 0.000738839 & 0.07936277 \\
\hline Commd10 & 13.07110695 & 12.22251811 & -0.848588842 & 0.000741977 & 0.07936277 \\
\hline Usp13_predicted & 8.880457276 & 8.301686706 & -0.57877057 & 0.000751682 & 0.07936277 \\
\hline TC539690 & 11.53252368 & 10.19001348 & -1.342510194 & 0.000754935 & 0.07936277 \\
\hline AW142620 & 10.54231861 & 9.200592376 & -1.341726234 & 0.000763691 & 0.079628835 \\
\hline Keap1 & 12.00883633 & 11.60287219 & -0.405964134 & 0.000792061 & 0.081218196 \\
\hline Gsk3b & 10.9550178 & 10.01148948 & -0.943528324 & 0.000797188 & 0.081294469 \\
\hline RGD1310192_predicted & 10.30348412 & 9.743106299 & -0.560377818 & 0.000800586 & 0.081294469 \\
\hline AW919892 & 9.009219476 & 8.398195424 & -0.611024052 & 0.00080164 & 0.081294469 \\
\hline CX570570 & 9.009092379 & 8.150761251 & -0.858331128 & 0.00081345 & 0.081955526 \\
\hline CO403204 & 7.341185431 & 6.396430715 & -0.944754716 & 0.000815344 & 0.081955526 \\
\hline DV727788 & 9.546149034 & 8.506229032 & -1.039920002 & 0.000820046 & 0.082157822 \\
\hline Crebl2 & 8.237847284 & 7.059695143 & -1.178152141 & 0.000832899 & 0.082830708 \\
\hline RGD1566078_predicted & 15.4991444 & 14.43680942 & -1.062334982 & 0.000843282 & 0.082831536 \\
\hline TC536252 & 6.791496764 & 6.081404474 & -0.71009229 & 0.000848504 & 0.082831536 \\
\hline RGD1310383_predicted & 9.675442727 & 8.875211356 & -0.800231371 & 0.000854032 & 0.082831536 \\
\hline Ndrg2 & 16.92933608 & 16.23958962 & -0.689746461 & 0.000855608 & 0.082831536 \\
\hline AA819653 & 6.401981606 & 5.54072971 & -0.861251897 & 0.000864825 & 0.082831536 \\
\hline $\mathrm{Nr} 1 \mathrm{~d} 2$ & 4.102851341 & 2.907566815 & -1.195284526 & 0.000870343 & 0.082831536 \\
\hline Al030552 & 6.242106917 & 4.813012178 & -1.429094739 & 0.000872853 & 0.082831536 \\
\hline TC557120 & 11.58985733 & 11.08039255 & -0.509464782 & 0.000878637 & 0.083021035 \\
\hline CB547657 & 7.11769739 & 5.41137132 & -1.706326069 & 0.000885415 & 0.083021035 \\
\hline TC551191 & 7.699104736 & 6.244599038 & -1.454505698 & 0.000887765 & 0.083021035 \\
\hline Aytl2_predicted & 7.999183956 & 7.427312229 & -0.571871727 & 0.000891151 & 0.083021035 \\
\hline Ddef2_predicted & 7.639303041 & 7.13151159 & -0.507791451 & 0.000898719 & 0.083047388 \\
\hline Ratsg2 & 11.68645688 & 11.30312412 & -0.383332762 & 0.000913048 & 0.083047388 \\
\hline ENSRNOTO0000043649 & 12.10622909 & 11.2676376 & -0.838591496 & 0.00093425 & 0.083047388 \\
\hline Slc4a4 & 10.52371038 & 9.660948829 & -0.862761553 & 0.000935657 & 0.083047388 \\
\hline Copb2 & 12.96384037 & 12.52074638 & -0.443093992 & 0.000945107 & 0.083047388 \\
\hline Plekhc1 & 9.749091344 & 9.407128148 & -0.341963196 & 0.000946928 & 0.083047388 \\
\hline RGD1561030_predicted & 5.157115548 & 4.180877924 & -0.976237624 & 0.000950336 & 0.083047388 \\
\hline TC525601 & 13.50942052 & 12.9991972 & -0.510223326 & 0.000950464 & 0.083047388 \\
\hline Phf7 & 8.191447181 & 7.356775616 & -0.834671565 & 0.000953909 & 0.083047388 \\
\hline BQ782721 & 4.908138931 & 4.310952068 & -0.597186864 & 0.000963823 & 0.083047388 \\
\hline AA925274 & 5.579121622 & 5.090008894 & -0.489112728 & 0.000969917 & 0.083047388 \\
\hline DV727304 & 11.16319859 & 10.59010722 & -0.573091369 & 0.000975685 & 0.083047388 \\
\hline
\end{tabular}


Table 4. List of DEGs after H2A.Z.1 and H2A.Z.2 KD-part C (upregulated after H2A.Z.2 KD)

\begin{tabular}{|c|c|c|c|c|c|}
\hline Symbol & Scrambled expression & shH2AZ.2 expression & log2 fold change & $p$ value & FDR \\
\hline Ahcyl1_predicted & 11.30812916 & 15.70257541 & 4.394446257 & $1.55 \mathrm{E}-09$ & $4.75 \mathrm{E}-05$ \\
\hline Mcm6 & 10.83536001 & 11.40618674 & 0.570826728 & $8.24 \mathrm{E}-06$ & 0.035135455 \\
\hline Dut & 11.35492304 & 11.9535574 & 0.598634355 & $3.46 \mathrm{E}-05$ & 0.066139598 \\
\hline Timp2 & 15.05728576 & 15.80164866 & 0.744362903 & $3.81 \mathrm{E}-05$ & 0.067378588 \\
\hline RGD1311752_predicted & 8.703882692 & 10.33537822 & 1.631495533 & $4.06 \mathrm{E}-05$ & 0.067378588 \\
\hline Zcwpw1_predicted & 8.549635796 & 9.169491306 & 0.619855511 & $4.50 \mathrm{E}-05$ & 0.067378588 \\
\hline XM_345006 & 6.85771805 & 7.739541418 & 0.881823368 & $4.87 \mathrm{E}-05$ & 0.067378588 \\
\hline TC549858 & 11.27224616 & 11.75359808 & 0.481351913 & $4.91 \mathrm{E}-05$ & 0.067378588 \\
\hline Hrasls_predicted & 1.352310448 & 2.473461424 & 1.121150976 & $5.02 \mathrm{E}-05$ & 0.067378588 \\
\hline BF289520 & 5.430397248 & 6.059021678 & 0.62862443 & $7.69 \mathrm{E}-05$ & 0.071367889 \\
\hline Pold3 & 10.06736193 & 10.48690247 & 0.419540544 & $7.71 \mathrm{E}-05$ & 0.071367889 \\
\hline RGD1307357_predicted & 11.2118082 & 11.5384726 & 0.326664395 & 7.97E-05 & 0.07161003 \\
\hline Gtf3c5_predicted & 7.76009444 & 8.355130115 & 0.595035675 & $9.19 \mathrm{E}-05$ & 0.073611113 \\
\hline Bcl7c_predicted & 11.52854784 & 12.20876021 & 0.68021237 & 0.000109679 & 0.073611113 \\
\hline Pomc & 8.615004399 & 9.436951714 & 0.821947315 & 0.000109978 & 0.073611113 \\
\hline RGD1565291_predicted & 12.39574606 & 12.90176243 & 0.506016372 & 0.000117613 & 0.074873201 \\
\hline BF542749 & 10.74023303 & 12.36932877 & 1.629095739 & 0.000127234 & 0.077757776 \\
\hline Abcc2 & 8.819186435 & 9.789025783 & 0.969839348 & 0.000139329 & 0.080329614 \\
\hline LOC363188 & 5.75509275 & 8.424133782 & 2.669041033 & 0.000147266 & 0.083333563 \\
\hline Cecr5_predicted & 10.95802065 & 11.33987144 & 0.381850792 & 0.000176782 & 0.08855644 \\
\hline AA955618 & 9.344852039 & 9.669319187 & 0.324467148 & 0.000209302 & 0.09181501 \\
\hline Al044097 & 5.588998691 & 6.267474587 & 0.678475896 & 0.000221456 & 0.092025775 \\
\hline RGD1563319_predicted & 8.12622631 & 9.457387834 & 1.331161525 & 0.000236732 & 0.092741286 \\
\hline Tbc1d2b & 6.869829194 & 8.039756988 & 1.169927794 & 0.000255978 & 0.095023281 \\
\hline ENSRNOT00000027501 & 9.948158576 & 10.60865914 & 0.660500565 & 0.000307587 & 0.095557268 \\
\hline RGD1304587 & 12.28837897 & 12.94341986 & 0.655040885 & 0.000314183 & 0.095557268 \\
\hline TC542265 & 9.893597595 & 10.95071148 & 1.05711388 & 0.000351139 & 0.095557268 \\
\hline ВМ384088 & 9.56848326 & 10.08580713 & 0.517323868 & 0.000375917 & 0.095557268 \\
\hline BG668164 & 9.868977302 & 10.4107215 & 0.541744198 & 0.000393414 & 0.095557268 \\
\hline Centg3_predicted & 12.84619462 & 13.64912229 & 0.802927674 & 0.000397418 & 0.095557268 \\
\hline $\mathrm{Rfc} 2$ & 10.69628045 & 11.25875805 & 0.562477599 & 0.000401566 & 0.095557268 \\
\hline Slc26a7_predicted & 4.035522477 & 6.194273087 & 2.15875061 & 0.000404596 & 0.095557268 \\
\hline Fn1 & 8.587931475 & 9.897009695 & 1.30907822 & 0.00041856 & 0.095557268 \\
\hline Fxyd6 & 15.52204053 & 16.22851939 & 0.706478863 & 0.000424885 & 0.095557268 \\
\hline Ezh1_predicted & 13.35214855 & 13.9436327 & 0.59148415 & 0.000436061 & 0.095557268 \\
\hline DV719732 & 9.123092282 & 9.737560979 & 0.614468697 & 0.000443541 & 0.095557268 \\
\hline B4galt7 & 12.13569399 & 12.61112377 & 0.475429774 & 0.000458424 & 0.095557268 \\
\hline Lzts2 & 10.13285103 & 10.82559939 & 0.692748367 & 0.000469953 & 0.095557268 \\
\hline Src & 9.695578825 & 10.36460234 & 0.669023515 & 0.000512904 & 0.095557268 \\
\hline BF556192 & 12.80155585 & 13.64456502 & 0.84300917 & 0.000526513 & 0.095557268 \\
\hline RGD1564308_predicted & 8.53728578 & 9.316997749 & 0.77971197 & 0.00053388 & 0.095557268 \\
\hline AW915160 & 11.81695687 & 12.84151282 & 1.024555952 & 0.000534083 & 0.095557268 \\
\hline BF289433 & 4.810359467 & 5.442835512 & 0.632476045 & 0.000539006 & 0.095557268 \\
\hline LOC312502 & 9.813073542 & 10.28343955 & 0.470366013 & 0.000543607 & 0.095557268 \\
\hline Slc35b4_predicted & 10.07187502 & 10.57533921 & 0.503464186 & 0.000547104 & 0.095557268 \\
\hline Ten2 & 8.702856424 & 9.597660836 & 0.894804412 & 0.000548566 & 0.095557268 \\
\hline Rabl4_predicted & 12.99354632 & 13.58209364 & 0.588547326 & 0.000558303 & 0.095557268 \\
\hline Astn1 & 7.130219184 & 7.783368618 & 0.653149434 & 0.000564477 & 0.095557268 \\
\hline Slitrk1_predicted & 11.38978024 & 11.86215613 & 0.47237589 & 0.000565343 & 0.095557268 \\
\hline LOC678926 & 1.529149853 & 3.301916023 & 1.77276617 & 0.000568879 & 0.095557268 \\
\hline Sfmbt1 & 10.57247665 & 11.52524221 & 0.952765564 & 0.000576444 & 0.095557268 \\
\hline Nfya & 7.339752471 & 8.230520214 & 0.890767743 & 0.000594435 & 0.095557268 \\
\hline CA503664 & 8.706063302 & 9.30753056 & 0.601467258 & 0.000612386 & 0.095557268 \\
\hline CO398332 & 2.088031423 & 3.465147675 & 1.377116252 & 0.000617647 & 0.095557268 \\
\hline Ankrd5_predicted & 0.986066003 & 1.893556424 & 0.907490421 & 0.000618681 & 0.095557268 \\
\hline RGD1561903_predicted & 8.073236386 & 9.261517734 & 1.188281348 & 0.000624827 & 0.095557268 \\
\hline Nav1_predicted & 9.311359188 & 10.05118484 & 0.739825648 & 0.000627604 & 0.095557268 \\
\hline Lonrf1_predicted & 6.665069586 & 8.231081607 & 1.566012021 & 0.000637379 & 0.095557268 \\
\hline LOC291840 & 10.46425437 & 10.98618406 & 0.521929682 & 0.00064038 & 0.095557268 \\
\hline BF555594 & 7.297901913 & 7.803911007 & 0.506009094 & 0.000642572 & 0.095557268 \\
\hline Prcp_predicted & 6.945396355 & 7.628166927 & 0.682770572 & 0.000650274 & 0.095557268 \\
\hline Cggbp1_predicted & 10.10446551 & $\begin{array}{r}10.67233084 \\
\text { (Continued) }\end{array}$ & 0.567865332 & 0.000651252 & 0.095557268 \\
\hline
\end{tabular}


Table 4. Continued

\begin{tabular}{|c|c|c|c|c|c|}
\hline Symbol & Scrambled expression & shH2AZ.2 expression & log2 fold change & $p$ value & FDR \\
\hline LOC686668 & 14.22879788 & 14.64664596 & 0.417848086 & 0.000653103 & 0.095557268 \\
\hline Shank1 & 10.5815021 & 11.85728804 & 1.275785942 & 0.000653256 & 0.095557268 \\
\hline $\mathrm{BI} 293610$ & 9.493734401 & 9.933127533 & 0.439393131 & 0.000667597 & 0.095557268 \\
\hline RGD1308637 & 10.63111621 & 10.99277702 & 0.361660808 & 0.000669694 & 0.095557268 \\
\hline LOC683646 & 14.6252591 & 15.00216313 & 0.376904029 & 0.000672344 & 0.095557268 \\
\hline TC562439 & 6.699930546 & 8.729363905 & 2.029433359 & 0.00068664 & 0.095625151 \\
\hline RGD1566063_predicted & 10.42408783 & 10.82360645 & 0.399518627 & 0.000691139 & 0.095625151 \\
\hline XM_214416 & 9.178834112 & 9.6187083 & 0.439874188 & 0.000699314 & 0.095625151 \\
\hline RGD1308557_predicted & 7.420320921 & 8.285465612 & 0.865144691 & 0.000733081 & 0.09725562 \\
\hline RGD1566292_predicted & 11.21332683 & 11.71063736 & 0.49731053 & 0.000751031 & 0.097309775 \\
\hline RGD1305166_predicted & 9.134297704 & 9.759187045 & 0.624889341 & 0.000751656 & 0.097309775 \\
\hline Nkiras2_predicted & 10.80772401 & 11.77210764 & 0.964383627 & 0.000753238 & 0.097309775 \\
\hline Cybasc3 & 7.043310663 & 7.609593274 & 0.566282611 & 0.000754734 & 0.097309775 \\
\hline Mrpl36_predicted & 11.89399742 & 12.33502107 & 0.441023645 & 0.000782062 & 0.098010329 \\
\hline Gprk2I & 8.779110352 & 9.38417973 & 0.605069378 & 0.00080672 & 0.098999718 \\
\hline LOC686766 & 5.260876485 & 5.911787655 & 0.65091117 & 0.000811953 & 0.099243336 \\
\hline Phf22 & 7.538016027 & 8.324087108 & 0.786071082 & 0.000836521 & 0.101357181 \\
\hline RGD1306143_predicted & 6.850673885 & 7.335015831 & 0.484341946 & 0.00084597 & 0.101533717 \\
\hline ENSRNOT00000048826 & 12.49598977 & 12.94226435 & 0.446274573 & 0.000847305 & 0.101533717 \\
\hline Znf579_predicted & 11.20811184 & 11.67550922 & 0.467397383 & 0.000856332 & 0.10181686 \\
\hline Hsdl1 & 9.808903868 & 10.14043298 & 0.331529114 & 0.000883112 & 0.102944694 \\
\hline Gprk6 & 12.47356751 & 13.25494457 & 0.781377066 & 0.0008894 & 0.102944694 \\
\hline Hsd17b2 & 3.414854355 & 5.901570573 & 2.486716218 & 0.000943101 & 0.104035619 \\
\hline Rnf8 & 12.29803729 & 12.87630368 & 0.578266392 & 0.000952234 & 0.104035619 \\
\hline CA510534 & 11.34797575 & 12.80389432 & 1.455918574 & 0.000958972 & 0.104035619 \\
\hline Arl2 & 10.91134235 & 11.45372552 & 0.542383164 & 0.000971266 & 0.104035619 \\
\hline BF550209 & 12.37525589 & 12.8730042 & 0.497748308 & 0.000974014 & 0.104035619 \\
\hline BG665133 & 11.20679244 & 12.33422623 & 1.127433798 & 0.000979781 & 0.104035619 \\
\hline LOC691075 & 9.060232543 & 9.478672438 & 0.418439896 & 0.000980214 & 0.104035619 \\
\hline RGD1308535_predicted & 9.816766494 & 10.28292832 & 0.46616183 & 0.000993396 & 0.104035619 \\
\hline Zswim5_predicted & 5.470774971 & 6.301634314 & 0.830859343 & 0.000996038 & 0.104035619 \\
\hline LOC690354 & 10.40224677 & 10.7558671 & 0.353620329 & 0.000997294 & 0.104035619 \\
\hline
\end{tabular}

Table 5. List of DEGs after H2A.Z.1 and H2A.Z.2 KD-part D (downregulated after H2A.Z.2 KD)

\begin{tabular}{|c|c|c|c|c|c|}
\hline Symbol & Scrambled expression & shH2AZ.2 expression & log2 fold change & $p$ value & FDR \\
\hline Rnf14 & 16.57094576 & 12.9463825 & -3.624563259 & $1.91 \mathrm{E}-08$ & 0.000291288 \\
\hline RGD1359108 & 13.30079953 & 7.879686571 & -5.421112956 & $4.85 \mathrm{E}-08$ & 0.000494164 \\
\hline Inpp5f_predicted & 4.824671522 & 3.588112893 & -1.236558629 & $1.13 \mathrm{E}-06$ & 0.008652283 \\
\hline Cfl2_predicted & 10.28475755 & 8.941614741 & -1.343142814 & $5.78 \mathrm{E}-06$ & 0.033552658 \\
\hline Tex2 & 6.820637729 & 5.774872776 & -1.045764953 & $6.59 \mathrm{E}-06$ & 0.033552658 \\
\hline Lypla3 & 10.13489074 & 9.305455152 & -0.829435583 & $9.20 \mathrm{E}-06$ & 0.035135455 \\
\hline Sbds & 10.29127274 & 9.876346931 & -0.414925805 & $1.21 \mathrm{E}-05$ & 0.041035544 \\
\hline Poldip2_predicted & 11.21978549 & 10.11515746 & -1.104628032 & $1.36 \mathrm{E}-05$ & 0.041433838 \\
\hline RGD1564626_predicted & 7.402821848 & 6.749656546 & -0.653165302 & $1.94 \mathrm{E}-05$ & 0.05398694 \\
\hline Chchd3_predicted & 13.15554854 & 11.50732751 & -1.648221032 & $2.44 \mathrm{E}-05$ & 0.062079366 \\
\hline Wee1 & 7.228033394 & 5.956374529 & -1.271658865 & $2.65 \mathrm{E}-05$ & 0.06223942 \\
\hline U78138 & 8.370979606 & 6.934655598 & -1.436324008 & $3.15 \mathrm{E}-05$ & 0.066139598 \\
\hline XM_343572 & 12.87985279 & 12.26365661 & -0.616196176 & $3.39 \mathrm{E}-05$ & 0.066139598 \\
\hline $\mathrm{Al} 231051$ & 5.91510764 & 4.336231214 & -1.578876425 & 5.07E-05 & 0.067378588 \\
\hline Gad2 & 7.493926937 & 5.159298815 & -2.334628122 & $5.51 \mathrm{E}-05$ & 0.06840792 \\
\hline Tmem55a & 10.29421099 & 9.510629962 & -0.783581025 & $5.80 \mathrm{E}-05$ & 0.06840792 \\
\hline Lsm14a_predicted & 8.399662619 & 7.415627465 & -0.984035154 & $6.05 \mathrm{E}-05$ & 0.068497749 \\
\hline LOC300429 & 6.310030902 & 5.753092672 & -0.556938229 & $6.85 \mathrm{E}-05$ & 0.071367889 \\
\hline Sdcbp2 & 3.853370711 & 3.05190314 & -0.801467571 & $6.94 \mathrm{E}-05$ & 0.071367889 \\
\hline LOC691853 & 9.783324367 & 8.799255704 & -0.984068662 & 7.16E-05 & 0.071367889 \\
\hline XM_215270 & 10.17499295 & 9.701596351 & -0.4733966 & $7.43 \mathrm{E}-05$ & 0.071367889 \\
\hline TC53-̄8215 & 9.067975732 & 7.252392924 & -1.815582808 & $8.86 \mathrm{E}-05$ & 0.073611113 \\
\hline BG663025 & 6.859758488 & $\begin{array}{l}5.74663244 \\
\text { (Continued) }\end{array}$ & -1.113126048 & $8.89 \mathrm{E}-05$ & 0.073611113 \\
\hline
\end{tabular}


Table 5. Continued

\begin{tabular}{|c|c|c|c|c|c|}
\hline Symbol & Scrambled expression & shH2AZ.2 expression & log2 fold change & $p$ value & FDR \\
\hline TC553657 & 8.723092234 & 8.029121219 & -0.693971015 & $9.42 \mathrm{E}-05$ & 0.073611113 \\
\hline LOC684322 & 15.44820244 & 14.34666049 & -1.101541943 & $9.84 \mathrm{E}-05$ & 0.073611113 \\
\hline Usp32_predicted & 10.25821098 & 9.517372161 & -0.74083882 & 0.000100946 & 0.073611113 \\
\hline ENSRNOT00000008889 & 8.21062512 & 6.810763402 & -1.399861718 & 0.000104197 & 0.073611113 \\
\hline $\mathrm{Rb} 1$ & 8.11762273 & 6.908923008 & -1.208699722 & 0.000105314 & 0.073611113 \\
\hline AA849985 & 6.116135406 & 5.241886237 & -0.874249169 & 0.000110254 & 0.073611113 \\
\hline AW917590 & 14.02219537 & 12.7498481 & -1.272347268 & 0.000111886 & 0.073611113 \\
\hline CB547899 & 7.72064826 & 7.029262406 & -0.691385855 & 0.000113222 & 0.073611113 \\
\hline U78132 & 7.279222721 & 5.846742231 & -1.43248049 & 0.000121025 & 0.075472833 \\
\hline Mcf2I & 9.397430132 & 8.657675863 & -0.739754268 & 0.00013013 & 0.077968283 \\
\hline DV713600 & 13.15808683 & 11.11117444 & -2.046912388 & 0.00013701 & 0.080329614 \\
\hline TC539285 & 9.30257925 & 8.414415448 & -0.888163802 & 0.000151429 & 0.084131469 \\
\hline BF555161 & 10.59364955 & 9.409913061 & -1.183736486 & 0.000167666 & 0.087621405 \\
\hline Cdk5rap1 & 10.22213249 & 9.608946578 & -0.61318591 & 0.000168425 & 0.087621405 \\
\hline C0399145 & 7.567286017 & 6.640966202 & -0.926319815 & 0.000169575 & 0.087621405 \\
\hline BF544403 & 7.874729806 & 6.432614592 & -1.442115214 & 0.000172048 & 0.087621405 \\
\hline RGD1308147 & 9.865899439 & 8.842423951 & -1.023475489 & 0.000183836 & 0.090604523 \\
\hline Arl10 & 7.253170158 & 6.41752538 & -0.835644778 & 0.000195782 & 0.09181501 \\
\hline Ddn & 11.23443915 & 9.493619755 & -1.740819393 & 0.00019651 & 0.09181501 \\
\hline AW535924 & 2.716704964 & 1.208805749 & -1.507899216 & 0.000200473 & 0.09181501 \\
\hline AW917511 & 7.921316801 & 7.098393914 & -0.822922887 & 0.000207115 & 0.09181501 \\
\hline Atxn1 & 7.381331792 & 6.174416218 & -1.206915574 & 0.000210514 & 0.09181501 \\
\hline Eaf2 & 5.106563436 & 4.649076352 & -0.457487084 & 0.000211747 & 0.09181501 \\
\hline TC557676 & 7.472657949 & 6.19779289 & -1.27486506 & 0.000212226 & 0.09181501 \\
\hline Ttc12 & 7.900994459 & 7.523648839 & -0.37734562 & 0.000213335 & 0.09181501 \\
\hline TC560921 & 5.207732953 & 4.559434897 & -0.648298056 & 0.000217244 & 0.092025775 \\
\hline Al112975 & 6.352713186 & 5.466730174 & -0.885983012 & 0.000228285 & 0.092025775 \\
\hline Vil2 & 9.860779156 & 8.967669681 & -0.893109474 & 0.000228882 & 0.092025775 \\
\hline AA875008 & 5.494818563 & 4.700887004 & -0.793931559 & 0.000235511 & 0.092741286 \\
\hline TC528454 & 8.35589654 & 6.885831687 & -1.470064854 & 0.000247959 & 0.09493033 \\
\hline Hspca & 14.42409811 & 13.70998635 & -0.714111765 & 0.00025058 & 0.09493033 \\
\hline Al059618 & 4.100124355 & 1.829444923 & -2.270679431 & 0.00025164 & 0.09493033 \\
\hline Rnf11_predicted & 10.2186121 & 9.615662304 & -0.602949799 & 0.000258106 & 0.095023281 \\
\hline Pole3 & 12.55132532 & 11.31822759 & -1.233097729 & 0.000262232 & 0.095273647 \\
\hline RGD1564451_predicted & 9.713981828 & 8.589317234 & -1.124664593 & 0.000265021 & 0.095273647 \\
\hline LOC683512 & 10.77887127 & 9.314774611 & -1.464096657 & 0.000269989 & 0.095557268 \\
\hline Stx 6 & 12.20386283 & 11.83254524 & -0.371317584 & 0.000275093 & 0.095557268 \\
\hline TC539851 & 11.07018722 & 9.38957993 & -1.680607294 & 0.0002773 & 0.095557268 \\
\hline RGD1308581_predicted & 11.33094265 & 10.731295 & -0.599647641 & 0.000301272 & 0.095557268 \\
\hline RGD1305052_predicted & 12.11367655 & 10.94947524 & -1.164201304 & 0.000303392 & 0.095557268 \\
\hline Pde4a & 9.320418187 & 8.426565098 & -0.893853089 & 0.000307772 & 0.095557268 \\
\hline RGD1565785_predicted & 3.454975278 & 1.716774529 & -1.738200749 & 0.000308741 & 0.095557268 \\
\hline TC551503 & 10.21507032 & 9.012702885 & -1.202367432 & 0.0003089 & 0.095557268 \\
\hline Sdhd & 14.37797406 & 13.64470284 & -0.733271224 & 0.00031574 & 0.095557268 \\
\hline AW144312 & 10.73124208 & 8.983532052 & -1.747710029 & 0.000316237 & 0.095557268 \\
\hline AW144351 & 9.197071628 & 7.899938415 & -1.297133212 & 0.000317995 & 0.095557268 \\
\hline Al599930 & 7.678810398 & 7.029318808 & -0.64949159 & 0.0003243 & 0.095557268 \\
\hline Ssu72 & 10.81988308 & 10.15157164 & -0.66831144 & 0.000326611 & 0.095557268 \\
\hline MGC125215 & 13.54599663 & 12.92503148 & -0.620965157 & 0.000330105 & 0.095557268 \\
\hline TC524569 & 8.967339631 & 8.237038714 & -0.730300917 & 0.000331746 & 0.095557268 \\
\hline Inpp4a & 8.581584747 & 7.638446802 & -0.943137945 & 0.000332111 & 0.095557268 \\
\hline TC540740 & 12.032179 & 11.48000894 & -0.552170063 & 0.000338226 & 0.095557268 \\
\hline TC550497 & 12.02106544 & 11.01957227 & -1.001493172 & 0.00034439 & 0.095557268 \\
\hline XM_580045 & 8.850520618 & 7.850512112 & -1.000008505 & 0.0003564 & 0.095557268 \\
\hline XM_236784 & 11.16551643 & 10.17544134 & -0.990075092 & 0.000365511 & 0.095557268 \\
\hline AW915589 & 9.355873567 & 7.887143897 & -1.46872967 & 0.000371323 & 0.095557268 \\
\hline Garnl1 & 6.899821493 & 6.075097502 & -0.824723991 & 0.00038265 & 0.095557268 \\
\hline H33079 & 11.05742197 & 10.63726213 & -0.420159837 & 0.000383244 & 0.095557268 \\
\hline LOC689577 & 10.30988195 & 9.693679501 & -0.616202449 & 0.000385184 & 0.095557268 \\
\hline LOC683630 & 9.849902817 & 9.088044225 & -0.761858591 & 0.000385627 & 0.095557268 \\
\hline Arpc5 & 14.5853404 & 13.66460753 & -0.920732865 & 0.000387329 & 0.095557268 \\
\hline RGD1305797_predicted & 5.300372467 & 4.356613034 & -0.943759433 & 0.000389433 & 0.095557268 \\
\hline TC546991 & 7.224586672 & $\begin{array}{l}5.935472523 \\
\text { (Continued) }\end{array}$ & -1.289114149 & 0.000393929 & 0.095557268 \\
\hline
\end{tabular}


Table 5. Continued

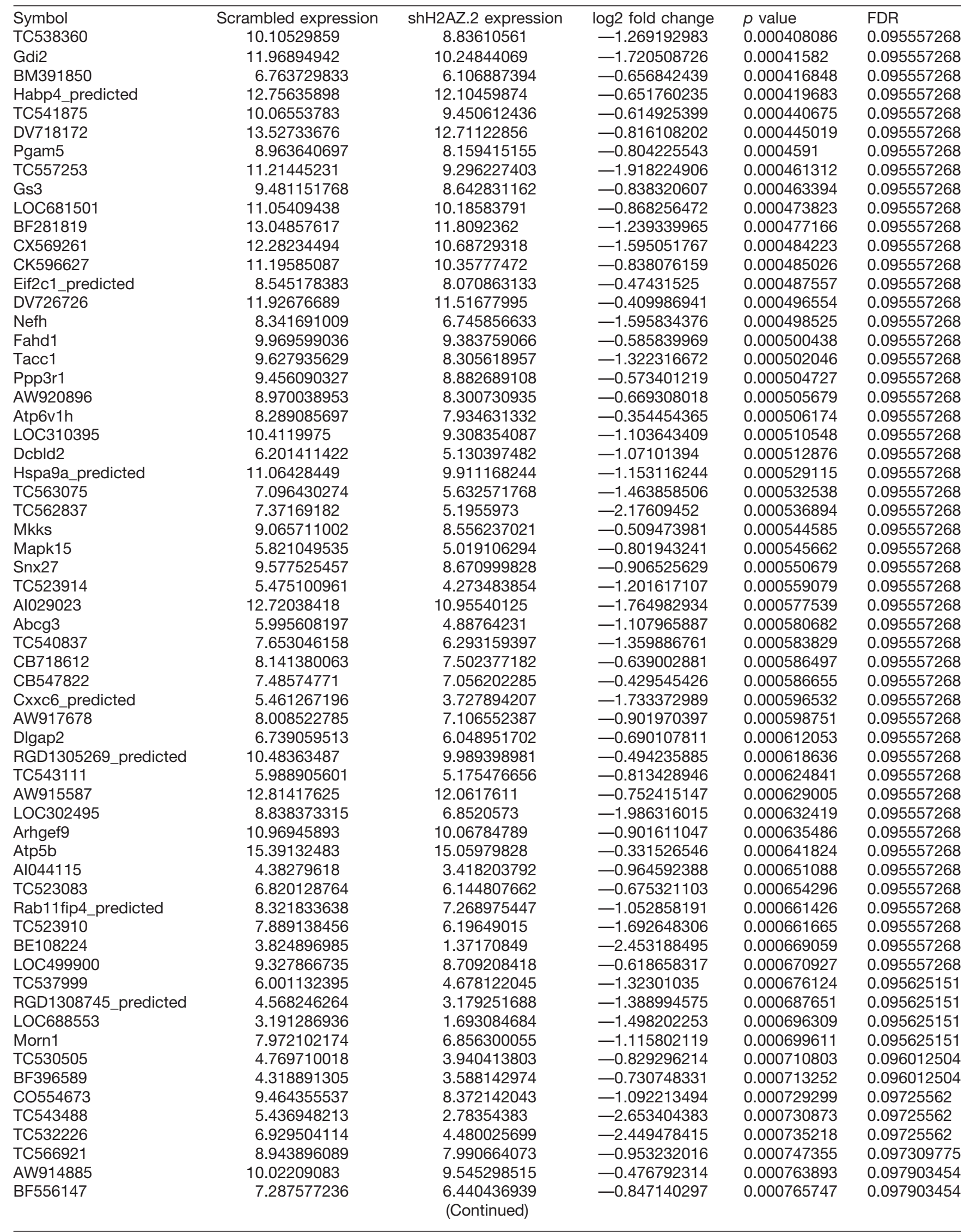


Table 5. Continued

\begin{tabular}{|c|c|c|c|c|c|}
\hline Symbol & Scrambled expression & shH2AZ.2 expression & log2 fold change & $p$ value & FDR \\
\hline Trim2 & 12.92583443 & 12.5169377 & -0.408896732 & 0.000769469 & 0.097969457 \\
\hline Gpr158_predicted & 6.695389757 & 5.535821733 & -1.159568025 & 0.000782843 & 0.098010329 \\
\hline BG666117 & 12.11075957 & 11.62393319 & -0.486826385 & 0.000784673 & 0.098010329 \\
\hline TC541992 & 7.227521264 & 6.312830078 & -0.914691186 & 0.000785637 & 0.098010329 \\
\hline TC525657 & 7.989864402 & 7.075628128 & -0.914236274 & 0.000787993 & 0.098010329 \\
\hline TC560235 & 8.131101996 & 7.601540913 & -0.529561083 & 0.000804594 & 0.098999718 \\
\hline LOC685076 & 11.93684157 & 10.40645356 & -1.53038801 & 0.000818584 & 0.099655287 \\
\hline AW143088 & 11.05925856 & 10.17888615 & -0.88037241 & 0.000852562 & 0.101764579 \\
\hline TC537691 & 11.03421255 & 10.1832052 & -0.851007351 & 0.000872934 & 0.102944694 \\
\hline Clcn2 & 10.18273395 & 9.654510577 & -0.528223376 & 0.000877455 & 0.102944694 \\
\hline DV719080 & 10.87559452 & 9.843234019 & -1.032360501 & 0.000877857 & 0.102944694 \\
\hline DY471780 & 7.546202473 & 6.688303944 & -0.857898528 & 0.000885155 & 0.102944694 \\
\hline Ppm1e & 8.969105775 & 7.850748852 & -1.118356922 & 0.000904556 & 0.103853197 \\
\hline TC538748 & 11.28808193 & 10.56735898 & -0.720722956 & 0.000907445 & 0.103853197 \\
\hline Al170363 & 4.98690796 & 4.240445668 & -0.746462292 & 0.000917269 & 0.104035619 \\
\hline TC523584 & 8.251731137 & 7.876311518 & -0.375419619 & 0.000921387 & 0.104035619 \\
\hline Al145492 & 4.960957943 & 3.550461441 & -1.410496501 & 0.000927701 & 0.104035619 \\
\hline CF109839 & 7.043330761 & 6.236742364 & -0.806588397 & 0.000929916 & 0.104035619 \\
\hline TC559053 & 8.399715145 & 7.440335331 & -0.959379814 & 0.000932371 & 0.104035619 \\
\hline AB040488 & 4.509816182 & 3.935696141 & -0.574120041 & 0.000936815 & 0.104035619 \\
\hline CB544681 & 7.403628364 & 6.79873552 & -0.604892844 & 0.000949976 & 0.104035619 \\
\hline Arhgef11 & 8.620841844 & 8.014495747 & -0.606346097 & 0.00096325 & 0.104035619 \\
\hline AA800571 & 8.989335176 & 8.145442592 & -0.843892585 & 0.000970797 & 0.104035619 \\
\hline AA965181 & 6.11182251 & 4.75033957 & -1.36148294 & 0.000977883 & 0.104035619 \\
\hline Ripk5 & 6.33665685 & 5.821877894 & -0.514778956 & 0.000978231 & 0.104035619 \\
\hline Phka1 & 5.544744433 & 4.356297924 & -1.188446509 & 0.000984497 & 0.104035619 \\
\hline TC524191 & 12.34462962 & 11.67308416 & -0.671545452 & 0.00098735 & 0.104035619 \\
\hline BG663084 & 9.722716915 & 8.835969466 & -0.886747449 & 0.000995922 & 0.104035619 \\
\hline BP503923 & 11.97203025 & 10.96018715 & -1.011843108 & 0.00099756 & 0.104035619 \\
\hline
\end{tabular}

\section{References}

Adelman K, Lis JT (2012) Promoter-proximal pausing of RNA polymerase II: emerging roles in metazoans. Nat Rev 13:720-731. CrossRef Medline

Alatwi HE, Downs JA (2015) Removal of H2A.Z by INO80 promotes homologous recombination. EMBO Rep 16:986-994. CrossRef Medline

Billon P, Cote J (2013) Precise deposition of histone H2A.Z in chromatin for genome expression and maintenance. Biochim Biophys Acta 1819:290-302.

Bruce K, Myers FA, Mantouvalou E, Lefevre P, Greaves I, Bonifer C, Tremethick DJ, Thorne AW, Crane-Robinson C (2005) The replacement histone H2A.Z in a hyperacetylated form is a feature of active genes in the chicken. Nucleic Acids Res 33:5633-5639. CrossRef

Chen Y, Stevens B, Chang J, Milbrandt J, Barres BA, Hell JW (2008) NS21: re-defined and modified supplement B27 for neuronal cultures. J Neurosci Methods 171:239-247. CrossRef Medline

Day DS, Zhang B, Stevens SM, Ferrari F, Larschan EN, Park PJ, Pu WT (2016) Comprehensive analysis of promoter-proximal RNA polymerase II pausing across mammalian cell types. Genome Biol 17:120-122. CrossRef Medline

Dryhurst D, Ishibashi T, Rose KL, Eirín-López JM, McDonald D, Silva-Moreno B, Veldhoen N, Helbing CC, Hendzel MJ, Shabanowitz J, Hunt DF, Ausió J (2009) Characterization of the histone H2A.Z-1 and H2A.Z-2 isoforms in vertebrates. BMC Biol 7:86. CrossRef Medline

Faast R, Thonglairoam V, Schulz TC, Beall J, Wells JR, Taylor H, Matthaei K, Rathjen PD, Tremethick DJ, Lyons I (2001) Histone variant $\mathrm{H} 2 \mathrm{~A} . \mathrm{Z}$ is required for early mammalian development. Curr Biol 11:1183-1187. Medline

Farris SD, Rubio ED, Moon JJ, Gombert WM, Nelson BH, Krumm A (2005) Transcription-induced chromatin remodeling at the c-myc gene involves the local exchange of histone H2A.Z. J Biol Chem 280:25298-25303. CrossRef Medline
Filipescu D, Szenker E, Almouzni G (2013) Developmental roles of histone H3 variants and their chaperones. Trends Genet 29:630640. CrossRef Medline

Geiss GK, Bumgarner RE, Birditt B, Dahl T, Dowidar N, Dunaway DL, Fell HP, Ferree S, George RD, Grogan T, James JJ, Maysuria M, Mitton JD, Oliveri P, Osborn JL, Peng T, Ratcliffe AL, Webster PJ, Davidson EH, Hood L, et al. (2008) Direct multiplexed measurement of gene expression with color-coded probe pairs. Nat Biotechnol 26:317-325. CrossRef Medline

Greer PL, Greenberg ME (2008) From synapse to nucleus: calcium-dependent gene transcription in the control of synapse development and function. Neuron 59:846-860. CrossRef Medline

Gursoy-Yuzugullu O, Ayrapetov MK, Price BD (2015) Histone chaperone Anp32e removes H2A.Z from DNA double-strand breaks and promotes nucleosome reorganization and DNA repair. Proc Natl Acad Sci USA 112:7507-7512. CrossRef Medline

Guzowski JF, McNaughton BL, Barnes CA, Worley PF (1999) Environment-specific expression of the immediate-early gene Arc in hippocampal neuronal ensembles. Nat Neurosci 2:11201124.

Horikoshi N, Sato K, Shimada K, Arimura Y, Osakabe A, Tachiwana H, Hayashi-Takanaka Y, Iwasaki W, Kagawa W, Harata M, Kimura $\mathrm{H}$, Kurumizaka H (2013) Structural polymorphism in the L1 loop regions of human H2A.Z.1 and H2A.Z.2. Acta Crystallogr D Biol Crystallogr 69:2431-2439. CrossRef Medline

Jimeno-González S, Ceballos-Chávez M, Reyes JC (2015) A positioned +1 nucleosome enhances promoter-proximal pausing. Nucleic Acids Res 43:3068-3078. CrossRef Medline

Kim TK, Hemberg M, Gray JM, Costa AM, Bear DM, Wu J, Harmin DA, Laptewicz M, Barbara-Haley K, Kuersten S, MarkenscoffPapadimitriou E, Kuhl D, Bito H, Worley PF, Kreiman G, Greenberg ME (2010) Widespread transcription at neuronal activity-regulated enhancers. Nature 465:182-187. CrossRef 
Komili S, Farny NG, Roth FP, Silver PA (2007) Functional specificity among ribosomal proteins regulates gene expression. Cell 131: 557-571. CrossRef Medline

Ku M, Jaffe JD, Koche RP, Rheinbay E, Endoh M, Koseki H, Carr SA, Bernstein BE (2012) H2A.Z landscapes and dual modifications in pluripotent and multipotent stem cells underlie complex genome regulatory functions. Genome Biol 13:R85. CrossRef

Kwak H, Lis JT (2013) Control of transcriptional elongation. Annu Rev Genet 47:483-508. CrossRef Medline

Lee K, Hsiung CC, Huang P, Raj A, Blobel GA (2015) Dynamic enhancer-gene body contacts during transcription elongation. Genes Dev 29:1992-1997. Medline

Mao Z, Pan L, Wang W, Sun J, Shan S, Dong Q, Liang X, Dai L, Ding X, Chen S, Zhang Z, Zhu B, Zhou Z (2014) Anp32e, a higher eukaryotic histone chaperone directs preferential recognition for H2A.Z. Cell Res 24:389-399. CrossRef Medline

Matsuda R, Hori T, Kitamura H, Takeuchi K, Fukagawa T, Harata M (2010) Identification and characterization of the two isoforms of the vertebrate H2A.Z histone variant. Nucleic Acids Res 38:42634273. CrossRef Medline

Maze I, Noh KM, Soshnev AA, Allis CD (2014) Every amino acid matters: essential contributions of histone variants to mammalian development and disease. Nat Rev 15:259-271. CrossRef Medline

Maze I, Wenderski W, Noh KM, Bagot RC, Tzavaras N, Purushothaman I, Elsässer SJ, Guo Y, lonete C, Hurd YL, Tamminga CA, Halene T, Farrelly L, Soshnev AA, Wen D, Rafii S, Birtwistle MR, Akbarian S, Buchholz BA, Blitzer RD, et al. (2015) Critical role of histone turnover in neuronal transcription and plasticity. Neuron 87:77-94. CrossRef Medline

Michod D, Bartesaghi S, Khelifi A, Bellodi C, Berliocchi L, Nicotera P, Salomoni P (2012) Calcium-dependent dephosphorylation of the histone chaperone DAXX regulates $\mathrm{H} 3.3$ loading and transcription upon neuronal activation. Neuron 74:122-135. CrossRef Medline

Obri A, Ouararhni K, Papin C, Diebold ML, Padmanabhan K, Marek M, Stoll I, Roy L, Reilly PT, Mak TW, Dimitrov S, Romier C, Hamiche A (2014) ANP32E is a histone chaperone that removes H2A.Z from chromatin. Nature 505:648-653. CrossRef Medline

Owen-Hughes T, Gkikopoulos T (2012) Making sense of transcribing chromatin. Curr Opin Cell Biol 24:296-304. CrossRef Medline

Papadia S, Stevenson P, Hardingham NR, Bading H, Hardingham GE (2005) Nuclear Ca2 + and the cAMP response element-binding protein family mediate a late phase of activity-dependent neuroprotection. J Neurosci 25:4279-4287. CrossRef Medline

Raisner RM, Madhani HD (2006) Patterning chromatin: form and function for H2A.Z variant nucleosomes. Curr Opin Genet Dev 16:119-124. CrossRef Medline

Rao VR, Pintchovski SA, Chin J, Peebles CL, Mitra S, Finkbeiner S (2006) AMPA receptors regulate transcription of the plasticityrelated immediate-early gene Arc. Nat Neurosci 9:887-895. Medline

Rosen LB, Ginty DD, Weber MJ, Greenberg ME (1994) Membrane depolarization and calcium influx stimulate MEK and MAP kinase via activation of Ras. Neuron 12:1207-1221. Medline

Saha RN, Dudek SM (2013) Splitting hares and tortoises: a classification of neuronal immediate early gene transcription based on poised RNA polymerase II. Neuroscience 247:175-181. CrossRef Medline

Saha RN, Wissink EM, Bailey ER, Zhao M, Fargo DC, Hwang JY, Daigle KR, Fenn JD, Adelman K, Dudek SM (2011) Rapid activity- induced transcription of Arc and other IEGs relies on poised RNA polymerase II. Nat Neurosci 14:848-856. CrossRef Medline

Schultz H, Engel K, Gaestel M (1997) PMA-induced activation of the p42/44ERK- and p38RK-MAP kinase cascades in HL-60 cells is PKC dependent but not essential for differentiation to the macrophage-like phenotype. J Cell Physiol 173:310-318. CrossRef Medline

Shechter D, Dormann HL, Allis CD, Hake SB (2007) Extraction, purification and analysis of histones. Nat Protoc 2:1445-1457. CrossRef Medline

Soboleva TA, Nekrasov M, Ryan DP, Tremethick DJ (2014) Histone variants at the transcription start-site. Trends Genet 30:199-209. CrossRef Medline

Steffen KK, McCormick MA, Pham KM, MacKay VL, Delaney JR, Murakami CJ, Kaeberlein M, Kennedy BK (2012) Ribosome deficiency protects against ER stress in Saccharomyces cerevisiae. Genetics 191:107-118. CrossRef Medline

Talbert PB, Ahmad K, Almouzni G, Ausió J, Berger F, Bhalla PL, Bonner WM, Cande WZ, Chadwick BP, Chan SW, Cross GA, Cui L, Dimitrov SI, Doenecke D, Eirin-López JM, Gorovsky MA, Hake SB, Hamkalo BA, Holec S, Jacobsen SE, et al. (2012) A unified phylogeny-based nomenclature for histone variants. Epigenetics Chromatin 5:7. CrossRef Medline

Teves SS, Weber CM, Henikoff S (2014) Transcribing through the nucleosome. Trends Biochem Sci 39:577-586. CrossRef Medline

Titov DV, Gilman B, He QL, Bhat S, Low WK, Dang Y, Smeaton M, Demain AL, Miller PS, Kugel JF, Goodrich JA, Liu JO (2011) XPB, a subunit of TFIIH, is a target of the natural product triptolide. Nat Chem Biol 7:182-188. Medline

Valdés-Mora F, Song JZ, Statham AL, Strbenac D, Robinson MD, Nair SS, Patterson KI, Tremethick DJ, Stirzaker C, Clark SJ (2012) Acetylation of H2A.Z is a key epigenetic modification associated with gene deregulation and epigenetic remodeling in cancer. Genome Res 22:307-321. CrossRef Medline

Vardabasso C, Gaspar-Maia A, Hasson D, Pünzeler S, Valle-Garcia D, Straub T, Keilhauer EC, Strub T, Dong J, Panda T, Chung CY, Yao JL, Singh R, Segura MF, Fontanals-Cirera B, Verma A, Mann M, Hernando E, Hake SB, Bernstein E (2015) Histone variant H2A.Z.2 mediates proliferation and drug sensitivity of malignant melanoma. Mol Cell 59:75-88. CrossRef Medline

Weber CM, Henikoff S (2014) Histone variants: dynamic punctuation in transcription. Genes Dev 28:672-682. CrossRef Medline

Wiegert JS, Bading H (2011) Activity-dependent calcium signaling and ERK-MAP kinases in neurons: a link to structural plasticity of the nucleus and gene transcription regulation. Cell Calcium 49: 296-305. CrossRef Medline

Wratting D, Thistlethwaite A, Harris M, Zeef LA, Millar CB (2012) A conserved function for the H2A.Z C terminus. J Biol Chem 287: 19148-19157. CrossRef Medline

Wu X, Brewer G (2012) The regulation of mRNA stability in mammalian cells: 2.0. Gene 500:10-21. CrossRef Medline

Yang Y, Yamada T, Hill KK, Hemberg M, Reddy NC, Cho HY, Guthrie AN, Oldenborg A, Heiney SA, Ohmae S, Medina JF, Holy TE, Bonni A (2016) Chromatin remodeling inactivates activity genes and regulates neural coding. Science 353:300-305. CrossRef Medline

Zlatanova J, Thakar A (2008) H2A.Z: view from the top. Structure 16:166-179. CrossRef Medline

Zovkic IB, Paulukaitis BS, Day JJ, Etikala DM, Sweatt JD (2014) Histone H2A.Z subunit exchange controls consolidation of recent and remote memory. Nature 515:582-586. CrossRef Medline 